\title{
Overview and Summary of the Second AIAA High Lift Prediction Workshop (Invited)
}

\author{
Christopher L. Rumsey* \\ NASA Langley Research Center, Hampton, VA 23681 \\ and \\ Jeffrey P. Slotnick ${ }^{\dagger}$ \\ The Boeing Company, Huntington Beach, CA 92647
}

The second AIAA CFD High-Lift Prediction Workshop was held in San Diego, California, in June 2013. The goals of the workshop continued in the tradition of the first high-lift workshop: to assess the numerical prediction capability of current-generation computational fluid dynamics (CFD) technology for swept, medium/high-aspect-ratio wings in landing/takeoff (high-lift) configurations. This workshop analyzed the flow over the DLR-F11 model in landing configuration at two different Reynolds numbers. Twenty-six participants submitted a total of $\mathbf{4 8}$ data sets of CFD results. A variety of grid systems (both structured and unstructured) were used. Trends due to grid density and Reynolds number were analyzed, and effects of support brackets were also included. This paper analyzes the combined results from all workshop participants. Comparisons with experimental data are made. A statistical summary of the CFD results is also included.

$\begin{array}{ll}\text { AR } & \text { aspect ratio } \\ \text { alpha, } \alpha & \text { angle of attack, deg } \\ B & \text { wing span } \\ C_{D} & \text { drag coefficient } \\ C_{D p} & \text { idealized drag coefficient }=C_{D}-C_{L}^{2} /(\pi A R) \\ C_{L} & \text { lift coefficient } \\ C_{L, m a x} & \text { maximum lift coefficient } \\ C_{M} & \text { pitching moment coefficient } \\ C_{f} & \text { surface skin friction coefficient } \\ C_{f, x} & x \text {-component of } C_{f} \\ C_{p} & \text { surface pressure coefficient } \\ C_{v} & \text { coefficient of variation }=\hat{\sigma} / \hat{\mu} \\ c & \text { mean aerodynamic chord } \\ h & \text { average grid spacing measure } \\ K & \text { confidence interval coverage factor } \\ \text { LB } & \text { lattice-Boltzmann } \\ M & \text { Mach number } \\ N & \text { number of grid points or grid cells } \\ R e & \text { Reynolds number } \\ R e_{c} & \text { Re based on } c \text { length scale } \\ u & \text { velocity component in the } x \text {-direction } \\ x, y, z & \text { Cartesian coordinate directions } \\ \Delta & \text { difference between high and low } R e \text { results } \\ \hat{\mu} & \text { median of sorted data } \\ \hat{\sigma} & \text { standard deviation } \\ & \end{array}$

\footnotetext{
*Senior Research Scientist, Computational AeroSciences Branch, Mail Stop 128, Fellow AIAA.

${ }^{\dagger}$ Boeing Technical Fellow, Computational Sciences, Senior Member AIAA.
} 


\section{Introduction}

The prediction of high-lift aerodynamic flows is a challenging but important aspect in the analysis of aircraft configurations. Today's computational fluid dynamics (CFD) codes have proven to be reliable and consistent for simplified, cruise-type configurations, but the complexities inherent in high-lift configurations add a significant degree of uncertainty. The complexities in high-lift flows include wakes in pressure gradients, wake/boundary-layer merging, streamline curvature, separated flow, possible unsteady flow, wing-tip vortical flow, and laminar/turbulent transition regions on each wing element (slat, main wing, flap). ${ }^{1}$

To advance the state of the art in predicting high-lift flows, an open international workshop series was established with the following long-term objectives: (1) assess the numerical prediction capability (mesh, numerics, turbulence modeling, high-performance computing requirements, etc.) of current-generation CFD technology for swept, medium/high-aspect ratio wings in landing/take-off (high lift) configurations, (2) develop practical modeling guidelines for CFD prediction of high lift flow fields, (3) advance the understanding of high lift flow physics to enable development of more accurate prediction methods and tools, (4) enhance CFD prediction capability for practical high lift aerodynamic design and optimization, (5) provide an impartial forum for evaluating the effectiveness of existing computer codes and modeling techniques, and (6) identify areas needing additional research and development.

Previously, the first high lift prediction workshop (HiLiftPW-1) was held in June 2010 in Chicago, Illinois. The workshop focused on the three-element NASA Trapezoidal Wing configuration ${ }^{2,3}$ at $R e_{c}=4.3 \times 10^{6}$; the workshop overview can be found in Slotnick et al., ${ }^{4}$ and the summary is given in Rumsey et al. ${ }^{5}$ The overall conclusions were as follows. CFD tended to under-predict lift, drag, and the magnitude of the pitching moment (pitching moment was negative) compared with experiment, and there was significantly more spread among the CFD solutions near stall. Despite the general under-prediction in lift, many participants were able to predict $C_{L, \max }$ and the angle of attack at which it occurred reasonably well. The trends with grid refinement were generally in the correct direction (approaching experiment), but it was difficult to draw firm conclusions because CFD grid convergence studies were only done without support brackets. Including support brackets resulted in lower predicted lift. Transition modeling, included by only a few participants, appeared to be very important for this configuration, tending to increase predicted lift. An unstructured tetrahedral grid solution was found to exhibit greater grid sensitivity than the solution on the same grid with its boundary-layer tetrahedra merged into prisms. The flow field near the wing tip was very difficult to predict. Nearly all entries seriously under-predicted the suction near the wing-tip upper surface, and neglecting viscous cross-derivative terms yielded even worse predictions near the wing tip than full Navier-Stokes. Statistically, the deltas predicted by CFD between two different configurations were somewhat low at $\alpha=13^{\circ}$, and significantly high at $\alpha=28^{\circ}$. Subsequently, many of the HiLiftPW-1 participants wrote papers highlighting their workshop results, ${ }^{6-19}$ and additional papers were produced for a later special session focused on the NASA Trapezoidal Wing at an AIAA meeting in June 2012 in New Orleans, Louisiana. ${ }^{20-32}$

The second high lift prediction workshop (HiLiftPW-2) was held in June 2013 in San Diego, California. ${ }^{a}$ The configuration selected for analysis was the DLR-F11 three-element wing-body model. ${ }^{33}$ This configuration was previously used in the EUROLIFT research program. ${ }^{34}$ The DLR-F11 was of interest because it was more representative of a realistic aircraft configuration than the NASA Trapezoidal Wing. Also, because the DLR-F11 was tested at both low and high Reynolds numbers, a major focus of HiLiftPW-2 was the ability of CFD to capture the Reynolds number effects.

This paper summarizes the HiLiftPW-2 workshop data, including several corrections and additional submissions by some participants soon after the workshop. A total of 26 groups submitted 48 entries. (This was slightly more than HiLiftPW-1, which had 21 groups and 39 entries.) In addition to describing the overall collective results in comparison to experimental data, an attempt is made in this paper to identify trends as well as outliers. In the interest of brevity, only representative results are shown in some cases.

The paper is organized as follows. First, the geometry and experimental data are briefly described, followed by a summary of the test cases and grid systems. A summary of the entries is then given. The results section includes a turbulence model verification test that was an optional exercise for the workshop participants, intended to help identify possible turbulence model implementation issues. Then, for the DLR-F11, the main results include force, pitching moment, grid convergence, iterative convergence, velocity, and surface pressure and skin friction coefficients. A statistical analysis is conducted in which scatter limits are calculated. As part of this statistical analysis, the ability to predict the differences between low and high Reynolds numbers is looked at in greater detail. Finally, conclusions are drawn.

${ }^{a}$ http://hiliftpw.larc.nasa.gov, accessed 11/13/2013. 


\section{Geometry and Experimental Data}

The DLR-F11 model—a generic semi-span, three element high lift wing configuration with a body pod—is shown in Fig. 1. It is intended to be representative of a commercial wide-body twin-jet high lift configuration, but the workshop focused on a simplified full-span flap geometry. The untwisted swept wing had a semi-span $(B / 2)$ of $1.4 \mathrm{~m}$ and an aspect ratio (AR) of 9.353 . The mean aerodynamic chord $(c)$ was $0.34709 \mathrm{~m}$. The high lift system consisted of a leading edge slat and a trailing edge Fowler flap, both full-span for the cases considered in this workshop. Transition was fixed on the body pod, but not on any of the wing elements. The slat was attached to the main wing using seven slat tracks. The flaps were mounted with five flap tracks and a fixing of the inner flap edge at the fuselage. The flap tracks were covered by flap track fairings. The high lift devices directly intersected with the fuselage. Due to the small size of the slat tracks, it was necessary to attach pressure tube bundles along the outside of the tracks for their routing into the slat element. Additional geometry and instrumentation details can be found in Rudnik et al. ${ }^{33}$ Note that throughout this paper, the slat tracks and flap track fairings are often collectively referred to as "support brackets" or "brackets."

The model was tested both at low Reynolds numbers in the low-speed wind tunnel Airbus-Deutschland (B-LSWT), as well as at high Reynolds numbers in the European Transonic Windtunnel (ETW). The wing was equipped with 487 pressure taps in ten chordwise pressure sections. In addition to forces, moments, and surface pressures, the low Reynolds number test included flow field velocity measurements using particle image velocimetry (PIV) and oil flow visualization. Other measurements not directly considered here included hot film, infared, and tuft videos. At $R e_{c}=1.35 \times 10^{6}, C_{L, \max }$ occurred near $\alpha=19^{\circ}$. Oil flow visualization at low Reynolds number suggested that at stall there were large isolated areas of separated flow over the main wing behind two of the slat tracks, spreading out laterally towards the trailing edge. In stall, the largest area of separated flow over the main wing was near midspan. Close to the flap trailing edge, separation trends were visible even at low angles of attack. The high Reynolds number test included forces, moments, and surface pressures. Tuft videos were made, but are not considered here. At $R e_{c}=15.1 \times 10^{6}, C_{L, \max }$ occurred near $\alpha=20-21^{\circ}$. Additional details about the experimental data, including short- and medium-term repeatability as well as facility effects, can be found in Rudnik et al. ${ }^{33}$

\section{Test Cases}

Like in HiLiftPW-1, the workshop test cases for HiLiftPW-2 were designed to generate enough quality CFD data so that meaningful solver-to-solver comparisons, as well as CFD comparisons with test data, could be conducted. The amount of data generated was extensive, including forces and moments, surface pressures and skin friction, velocity profiles, and both grid convergence and iterative convergence data. All DLR-F11 cases were in one particular landing configuration (slat $26.5^{\circ}$, flap $32^{\circ}$ ). The geometry was approximated in a series of configurations that built up the model for improved geometric fidelity:

- Config 2 - wing/body/high lift system plus side-of-body flap seal;

- Config 4 - same as Config 2 plus slat and flap support brackets;

- Config 5 - same as Config 4 plus slat pressure tube bundles

There were four test cases defined for the workshop. Three of these involved DLR-F11 Config 2, 4, or 5. The fourth test case was a simple verification exercise intended to isolate potential inconsistencies in turbulence model implementations. For the DLR-F11, the participants were asked to run on at least one of the grid series (detailed in the next section) supplied by the HiLiftPW committee. They were also allowed to create their own grids. In all cases, no wind tunnel walls or model support systems were to be included. The high Reynolds number conditions (corresponding to ETW) were $R e_{c}=15.1 \times 10^{6}, M=0.175, T_{r e f}=114.0 \mathrm{~K}, p_{r e f}=295 \mathrm{kPa}$. The low Reynolds number conditions (corresponding to B-LSWT) were $R e_{c}=1.35 \times 10^{6}, M=0.175, T_{r e f}=298.6 \mathrm{~K}, p_{\text {ref }}=100.7$ $\mathrm{kPa}$. The four test cases for the workshop are summarized here:

CASE 1 (required): Grid Convergence Study on Config 2 at high Reynolds number conditions

- $\alpha=7^{\circ}, 16^{\circ}$, (optional $\left.18.5^{\circ}\right)$;

- Coarse, Medium, and Fine grid solutions are required (Extra-Fine optional);

- run fully turbulent

CASE 2: Reynolds Number Study on Config 4, using Medium grid density

- Case 2a (required) - low Reynolds number conditions;

- $\alpha=0^{\circ}, 7^{\circ}, 12^{\circ}, 16^{\circ}, 18.5^{\circ}, 19^{\circ}, 20^{\circ}, 21^{\circ}$;

- run fully turbulent 
- Case $2 \mathrm{~b}$ (required) - high Reynolds number conditions;

- $\alpha=0^{\circ}, 7^{\circ}, 12^{\circ}, 16^{\circ}, 18.5^{\circ}, 20^{\circ}, 21^{\circ}, 22.4^{\circ}$;

- run fully turbulent

- Case 2c (optional) - low Reynolds number conditions;

- $\alpha=0^{\circ}, 7^{\circ}, 12^{\circ}, 16^{\circ}, 18.5^{\circ}, 19^{\circ}, 20^{\circ}, 21^{\circ}$;

- run with specified transition and/or transition prediction methods

CASE 3: Full Configuration Study on Config 5, using Medium grid density

- Case 3a (optional) - low Reynolds number conditions;

- $\alpha=0^{\circ}, 7^{\circ}, 12^{\circ}, 16^{\circ}, 18.5^{\circ}, 19^{\circ}, 20^{\circ}, 21^{\circ}$;

- run fully turbulent and/or run with transition

- Case 3b (optional) - high Reynolds number conditions;

- $\alpha=0^{\circ}, 7^{\circ}, 12^{\circ}, 16^{\circ}, 18.5^{\circ}, 20^{\circ}, 21^{\circ}, 22.4^{\circ}$;

- run fully turbulent and/or run with transition

CASE 4 (optional): Turbulence Model Verification Study

- 2-D bump case from http://turbmodels.larc.nasa.gov;

- $M=0.2, R e=3$ million per unit length;

- grid convergence study (at least 3 finest grid levels)

\section{Grid Systems}

For Case 1, five different DLR-F11 grid systems were supplied by the HiLiftPW committee, as summarized in Table 1 and Fig. 2. Grids for Case 2 were provided at the medium level for B, C, D, and E, and grids for Case 3 were provided at the medium level for $\mathrm{B}$ and $\mathrm{D}$, with $\mathrm{E}$ added several months after the workshop. The committee requested that all grids be developed according to a best-practice guideline published to the workshop website (noted in an earlier footnote). For brevity, only a few of the guidelines are summarized here; details can be found on the workshop website.

Each Case 1 grid system family was expected to supply at least three grids differing by levels of global refinement. At a minimum, coarse, medium, and fine grids were required with a nominal growth rate of 3 in total number of grid points or cells between one level and the next finest level. The far field was to be located at a distance of approximately 100 reference chords (i.e., 100c) for all grid levels.

The $\mathrm{C}$ and $\mathrm{D}$ grids were both constructed with tetrahedral elements; these were also subsequently merged to create mixed element grids with prism elements in the boundary layers. As described later, all participants who made use of these grids employed the mixed versions. Note that the block structured grid system A was also translated into unstructured hexahedral grids.

\section{Summary of Entries}

A summary of the entries to the HiLiftPW-2 is given in Tables 2 and 3. It includes an identifier for each submission, the code used, the code type (structured or unstructured), the grid system, the turbulence model, and other relevant information. All submissions were assigned an entry number, which consists of a primary number in front of the decimal point that identifies the group or person submitting the data, and a secondary sequence after the decimal point that identifies variations (for example, different grids, codes, or turbulence models). There were 26 individuals/groups who submitted 48 entries. The primary numbers are not sequential because there were three groups who dropped out prior to the workshop. Note that the structured codes that used grid system A employed point-match blocking, whereas those that used grid system E employed overset.

For turbulence models, most participants used the Spalart-Allmaras (SA) model ${ }^{35}$ or variant. Five of the variants are rotation/curvature (RC), ${ }^{36}$ rotation $(\mathrm{R}),{ }^{37}$ Edwards, ${ }^{38} \mathrm{SALSA},{ }^{39}$ and quadratic constitutive relation $(\mathrm{QCR}){ }^{40}$ The so-called "noft2" variant simply ignores the $f_{t 2}$ term in the turbulence model equation, which is believed to make very 
Table 1. Summary of supplied grids for Case 1 (number of grid points/cells given in millions)

\begin{tabular}{|c|c|c|c|c|c|c|c|c|}
\hline Label & $\begin{array}{l}\text { Grid } \\
\text { Tool }\end{array}$ & $\begin{array}{l}\text { Responsible } \\
\text { Org }\end{array}$ & Type & $\begin{array}{l}\text { Coarse } \\
\text { pts / cells }\end{array}$ & $\begin{array}{l}\text { Medium } \\
\text { pts / cells }\end{array}$ & $\begin{array}{l}\text { Fine } \\
\text { pts / cells }\end{array}$ & $\begin{array}{l}\text { Extra-Fine } \\
\text { pts / cells }\end{array}$ & Notes \\
\hline \multirow[t]{2}{*}{ A } & ICEM CFD & Boeing & str & $11 / 10$ & $34 / 32$ & $105 / 101$ & $\mathrm{n} / \mathrm{a}$ & $\begin{array}{l}\text { point-matched, } \\
\text { no brackets }\end{array}$ \\
\hline & & & unstr & $10 / 10$ & $32 / 32$ & $101 / 101$ & $\mathrm{n} / \mathrm{a}$ & hexahedra \\
\hline B & SOLAR & DLR & unstr & $9 / 21$ & $26 / 59$ & $73 / 165$ & $\mathrm{n} / \mathrm{a}$ & mixed \\
\hline \multirow[t]{2}{*}{$\mathrm{C}$} & Pointwise & Pointwise & unstr & $20 / 44$ & $59 / 133$ & $177 / 417$ & $\mathrm{n} / \mathrm{a}$ & mixed \\
\hline & & & & $20 / 117$ & $59 / 349$ & $177 / 1053$ & $\mathrm{n} / \mathrm{a}$ & tetrahedra \\
\hline \multirow[t]{2}{*}{ D } & VGRID & UWyo \& & unstr & $10 / 29$ & $31 / 99$ & $76 / 274$ & $\mathrm{n} / \mathrm{a}$ & mixed \\
\hline & & Cessna & & $10 / 60$ & $31 / 181$ & $76 / 447$ & $\mathrm{n} / \mathrm{a}$ & tetrahedra \\
\hline E & misc. & Boeing & str & $29 / 28$ & $69 / 67$ & $231 / 226$ & $545 / 537$ & overset \\
\hline
\end{tabular}

little difference. ${ }^{\mathrm{b}}$ Other models utilized by participants were $\mathrm{SST},{ }^{41} \mathrm{SST}-\mathrm{V},{ }^{42} \mathrm{~K}-\mathrm{e}-\mathrm{Rt},{ }^{43} \mathrm{EARSM},{ }^{44}$ and Wilcox $1988 .{ }^{45}$ There were also several non-traditional (not Reynolds-averaged Navier-Stokes) methods. The UNICORN code, a general Galerkin adaptive finite element method, used an implicit subgrid scale (SGS) model by numerical stabilization. The PowerFLOW code, a lattice-Boltzmann (LB) method, used a proprietary very large eddy simulation (VLES) wall model. The XFlow lattice-Boltzmann code used a proprietary wall-modeled local-eddy simulation (WMLES) model. For transition, an amplification factor transport (AFT) model,${ }^{46}$ the SST-GRET model, ${ }^{47}$ and a proprietary transition method (in PowerFLOW) were used.

\section{Results}

\section{A. Turbulence Modeling Verification}

An optional verification exercise (Case 4) was included in HiLiftPW-2 in part because a similar exercise had previously proved to be very useful for identifying discrepancies in turbulence modeling implementations at the fifth drag prediction workshop (DPW-5). ${ }^{48}$ Here, a simple 2-D bump case was used. At the website http://turbmodels.larc.nasa.gov, several different independent CFD codes (some of which have been previously verified) using the SA, SST, SST-V, and Wilcox2006 models have been shown to produce nearly identical results for this case as the grid is refined. Thus, the website has established high confidence in a grid-converged result for each of these models for this case. Any other code should expect to approach the same answer as the grid is refined, when using the same turbulence model and boundary conditions. A different solution indicates either an incorrect implementation of the model, an incorrect procedure or boundary condition, or a bug in the code. For example, in DPW-5, it was learned that inaccurate computation of the minimum distance function could lead to significant errors in SA results.

There were ten Case 4 submissions in HiLiftPW-2. Eight of these were for the SA model (or variant). Figure 3 shows the SA and SA variant results. In Fig. 3(a), the $C_{f}$ over a portion of the bump is shown. Three of the results were indistinguishable: 002.1,002.2, and 013.1. (Note that 002.1 and 002.2 corresponded to results already posted on the website http://turbmodels.larc.nasa.gov.) Of the other results, 007.1, 007.2, and 011.1 were slightly different, and the model variants 011.2 (SA-Edwards) and 011.3 (SA-salsa) were significantly different.

Comparing like-models only (SA) in the grid convergence study of Fig. 3(b), one can see that indeed 002.1, 002.2, and 013.1 were all approaching the same $C_{f}$ at location $x=0.6322$ as the grid was refined $\left(h^{2} \rightarrow 0\right)$. Submission 007.1 was only slightly low, but 007.2 was $2 \%$ low and 011.1 was about $3 \%$ high in this particular metric. Comparing $C_{f}$ at other locations yielded similar results. This study indicates that there are potential problems or inconsistencies in the implementation of the SA model in 007.2 and 011.1 that should be investigated.

The other two Case 4 submissions were 010.1 (K-e-Rt) and 018 (SST). The former currently has no other code to compare against for Case 4, and the latter used a different turbulence model for its Cases 1 and 4. Therefore, these results are not shown here.

\footnotetext{
${ }^{\mathrm{b}}$ See http://turbmodels.larc.nasa.gov, accessed 11/13/2013.
} 
Table 2. Summary of entries

\begin{tabular}{|c|c|c|c|c|c|c|}
\hline $\begin{array}{l}\text { Entry } \\
\text { Number }\end{array}$ & $\begin{array}{l}\text { Code } \\
\text { Name }\end{array}$ & Type & $\begin{array}{l}\text { Grid } \\
\text { System }\end{array}$ & $\begin{array}{l}\text { Turb } \\
\text { Model }\end{array}$ & Cases run & $\begin{array}{l}\text { ITER } \\
\text { provided? }\end{array}$ \\
\hline 002.1 & FUN3D & unstr & $\mathrm{D}$ (mixed) & SA & $\mathrm{C} 1, \mathrm{C} 2 \mathrm{a}, \mathrm{C} 2 \mathrm{~b}, \mathrm{C} 4$ & $\mathrm{Y}$ \\
\hline 002.2 & CFL3D & str & A & SA & $\mathrm{C} 1, \mathrm{C} 4$ & $\mathrm{Y}$ \\
\hline $003.1+$ & OVERFLOW & str & $\mathrm{E}$ & $\mathrm{SA}, \mathrm{SA}+\mathrm{AFT}$ & $\mathrm{C} 1, \mathrm{C} 2 \mathrm{a}, \mathrm{C} 2 \mathrm{~b}, \mathrm{C} 2 \mathrm{c}$ (trans) & $\mathrm{N}$ \\
\hline 003.2 & OVERFLOW & str & $\mathrm{E}$ & SA-RC & $\mathrm{C} 1, \mathrm{C} 2 \mathrm{a}, \mathrm{C} 2 \mathrm{~b}$ & $\mathrm{~N}$ \\
\hline $003.3+$ & OVERFLOW & str & $\mathrm{E}$ & SA-QCR+AFT & $\mathrm{C} 2 \mathrm{c}($ trans) only & $\mathrm{n} / \mathrm{a}$ \\
\hline 004.1 & $\mathrm{CFD}++$ & unstr & $\mathrm{B}+\mathrm{nc}$ & SA-RC & $\mathrm{C} 1$ only & $\mathrm{Y}$ \\
\hline $004.2 *$ & $\mathrm{CFD}++$ & unstr & $\mathrm{C}+\mathrm{nc}$ & SA-RC & C2b only & $\mathrm{n} / \mathrm{a}$ \\
\hline 005.1 & HiFUN & unstr & A & SA & $\mathrm{C} 1$ only & $\mathrm{Y}$ \\
\hline $005.2+$ & HiFUN & unstr & B & SA & $\mathrm{C} 1, \mathrm{C} 2 \mathrm{a}, \mathrm{C} 2 \mathrm{~b}$ & $\mathrm{Y}$ \\
\hline 006 & FUN3D & unstr & $\mathrm{D}$ (mixed) & SA-RC & $\mathrm{C} 1$ only & $\mathrm{Y}$ \\
\hline $007.1+$ & CFLOW & unstr & $\mathrm{C}($ mixed $)$ & SA & $\mathrm{C} 1, \mathrm{C} 2 \mathrm{a}, \mathrm{C} 2 \mathrm{~b}, \mathrm{C} 4$ & $\mathrm{Y}$ \\
\hline $007.2+$ & UG3 & unstr & $\mathrm{C}($ mixed $)$ & SA & $\mathrm{C} 1, \mathrm{C} 2 \mathrm{a}, \mathrm{C} 2 \mathrm{~b}, \mathrm{C} 4$ & $\mathrm{Y}$ \\
\hline $007.3^{*}$ & CFLOW & unstr & $\mathrm{nc}$ & SA & $\mathrm{C} 1$ only & $\mathrm{Y}$ \\
\hline 008 & CRUNCH CFD & unstr & $\mathrm{D}$ (mixed) & SST & $\mathrm{C} 1, \mathrm{C} 2 \mathrm{a}, \mathrm{C} 2 \mathrm{~b}$ & $\mathrm{~N}$ \\
\hline $009.1+$ & OVERFLOW & str & E & SA & $\mathrm{C} 1, \mathrm{C} 2 \mathrm{a}, \mathrm{C} 2 \mathrm{~b}$ & $\mathrm{Y}$ \\
\hline $009.2^{*}$ & OVERFLOW & str & $\mathrm{E}$ & SST & C2a only & $\mathrm{n} / \mathrm{a}$ \\
\hline $009.3^{*}$ & OVERFLOW & str & $\mathrm{E}$ & SST-GRET & $\mathrm{C} 2 \mathrm{c}$ (trans) only & $\mathrm{n} / \mathrm{a}$ \\
\hline $010.1+$ & $\mathrm{CFD}++$ & unstr & $A+C($ mixed $)$ & SA & $\mathrm{C} 1, \mathrm{C} 2 \mathrm{a}, \mathrm{C} 2 \mathrm{~b}$ & $\mathrm{Y}$ \\
\hline $010.2+$ & $\mathrm{CFD}++$ & unstr & A & SST & $\mathrm{C} 1$ only & $\mathrm{Y}$ \\
\hline $010.3+$ & $\mathrm{CFD}++$ & unstr & A & K-e-Rt & $\mathrm{C} 1, \mathrm{C} 4$ & $\mathrm{Y}$ \\
\hline $011.1+$ & NSMB & str & A & SA & $\mathrm{C} 1, \mathrm{C} 4$ & $\mathrm{Y}$ \\
\hline $011.2+$ & NSMB & str & A & SA-Edwards & $\mathrm{C} 1, \mathrm{C} 4$ & $\mathrm{Y}$ \\
\hline $011.3^{*}$ & NSMB & str & A & SA-salsa & $\mathrm{C} 1, \mathrm{C} 4$ & $\mathrm{Y}$ \\
\hline 012.1 & PowerFLOW & LB & $\mathrm{nc}$ & LBM-VLES & $\mathrm{C} 3 \mathrm{a}, \mathrm{C} 3 \mathrm{~b}$ & $\mathrm{n} / \mathrm{a}$ \\
\hline $012.2 *$ & PowerFLOW & LB & $\mathrm{nc}$ & LBM-VLES+trans & C3a(trans) only & $\mathrm{n} / \mathrm{a}$ \\
\hline $013.1+$ & UPACS & str & A & SA-noft2-R & $\mathrm{C} 1, \mathrm{C} 4$ & $\mathrm{Y}$ \\
\hline $013.2 *$ & UPACS & str & A & SA-noft2-R-QCR & $\mathrm{C} 1$ only & $\mathrm{Y}$ \\
\hline $013.3^{*}$ & UPACS & str & A & SST-V & $\mathrm{C} 1$ only & $\mathrm{Y}$ \\
\hline $013.4^{*}$ & UPACS & str & A & SST-V-QCR & $\mathrm{C} 1$ only & $\mathrm{Y}$ \\
\hline $013.5^{*}$ & TAS & unstr & $\mathrm{D}$ (mixed) & SA-noft2-R & $\mathrm{C} 1$ only & $\mathrm{Y}$ \\
\hline $013.6^{*}$ & TAS & unstr & $\mathrm{D}$ (mixed) & SA-noft2-R-QCR & $\mathrm{C} 1$ only & $\mathrm{N}$ \\
\hline 014 & UNICORN & unstr & $\mathrm{nc}$ & SGS & Adapt, C2b only & $\mathrm{n} / \mathrm{a}$ \\
\hline $015.1+$ & CFX & unstr & B & SST & $\mathrm{C} 1, \mathrm{C} 2 \mathrm{a}$ (no brk), $\mathrm{C} 2 \mathrm{~b}$ (no brk) & $\mathrm{N}$ \\
\hline $015.2+$ & Fluent & unstr & B & SST & $\mathrm{C} 1, \mathrm{C} 2 \mathrm{a}($ no brk), $\mathrm{C} 2 \mathrm{~b}$ (no brk) & $\mathrm{N}$ \\
\hline $016.1+$ & elsA & str & $\mathrm{E}$ & SA & $\mathrm{C} 1$ only & $\mathrm{N}$ \\
\hline $016.2^{*}$ & elsA & str & A & SA & $\mathrm{C} 1$ only & $\mathrm{Y}$ \\
\hline $017+$ & COBRA & str & $\mathrm{nc}$ & SA & $\mathrm{C} 1, \mathrm{C} 2 \mathrm{a}, \mathrm{C} 2 \mathrm{~b}$ & $\mathrm{~N}$ \\
\hline 018 & VULCAN & str & A & Wilcox 1988 & $\mathrm{C} 1, \mathrm{C} 4$ with SST & $\mathrm{N}$ \\
\hline
\end{tabular}

$*$ = new submission added after workshop

$+=$ modified after workshop

$\mathrm{nc}=$ non-committee grid

ITER $=$ iterative convergence history for $\mathrm{C} 1$ 
Table 3. Summary of entries (continued)

\begin{tabular}{lllllll}
\hline $\begin{array}{l}\text { Entry } \\
\text { Number }\end{array}$ & Code & Tyme & $\begin{array}{l}\text { Grid } \\
\text { System }\end{array}$ & $\begin{array}{l}\text { Turb } \\
\text { Model }\end{array}$ & Cases run & $\begin{array}{l}\text { ITER } \\
\text { provided? }\end{array}$ \\
\hline 020 & OVERFLOW & str & E & SA-noft2-RC & C1, C2a, C2b & Y \\
$021+$ & TAU & unstr & B & SA & C1, C2a, C2b, C3a, C3b & N \\
$022+$ & PHASTA & unstr & nc & SA & C1, C2a, C2b & Y \\
023 & NSU3D & unstr & D(mixed) & SA & C1, C2a, C2b & Y \\
024.1 & EDGE & unstr & B & EARSM & C1, C2a, C2b & Y \\
$024.2+$ & EDGE & unstr & B & SA & C1, C2a, C2b & Y \\
025 & NSU3D & unstr & nc & Wilcox1988 & C1, C2a, C2b & N \\
026 & MFLOW & str & A & SA & C1, C2a, C2b, C3a, C3b & N \\
028 & Fluent & unstr & A + B & SA-RC & C1 only & N \\
$029+$ & XFlow & LB & nc & WMLES & C1, C2a, C2b, C3b & N \\
\hline
\end{tabular}

$+=$ modified after workshop

$\mathrm{nc}=$ non-committee grid

ITER = iterative convergence history for $\mathrm{C} 1$

\section{B. Force and Moment Predictions}

\section{Case 1: Simplified Configuration (No Brackets)}

Figure 4 shows forces and pitching moments for Case 1 (CFD with no brackets) on the medium and fine grids. The lines are color-coded according to the type of method (black for unstructured, red for block-structured, green for overset, pink for lattice-Boltzmann). Experimental data are included for reference. Note that many participants did not complete all required cases, so there are some gaps and/or missing angles of attack in the plots. Some participants also included variation/uncertainty information when their result did not settle down to a constant value. This is shown as error bars in the plots.

Some of the outliers (results that stand out from the collective) are highlighted (011.1, 011.3, and 015.1). Ignoring these outliers, whose predicted lift tended to be lower than the collective, most participants predicted lift levels in good agreement with experiment up through about $\alpha=16^{\circ}$ and over-predicted lift at higher angles of attack. In fact, many participants did not predict stall even as high as $\alpha=22.4^{\circ}$. However, this is very likely a consequence of the fact that the brackets were not included in the computations for Case 1. Note that participants predicting lower lift tended to report higher uncertainty levels. In general, drag tended to be over-predicted for a given $C_{L}$ and pitching moment was typically predicted to be too negative (pitch down).

Grid convergence plots for the simplified configuration are shown in Figs. 5 and 6, for angles of attack of $\alpha=7^{\circ}$, $16^{\circ}, 18.5^{\circ}$, and $20^{\circ}$. Fewer participants performed grid studies at the higher angles of attack. In these plots, the level of $C_{L}, C_{D}$, or $C_{M}$ is plotted as a function of $N^{-2 / 3}$, which in 3-D is proportional to $h^{2}$. Finer grid results are on the left of the plots and coarser grid results are on the right. An infinitely fine grid would be plotted as $N^{-2 / 3}=0$. The corresponding experimental value is shown as a circle symbol on the y-axis of each plot for reference. If all codes and implementations were completely consistent and if all turbulence models were the same, then the band of results should tighten, approaching a single result as $N^{-2 / 3} \rightarrow 0$. This did not occur here. Overall, the scatter range remained more or less the same across the different grid levels. One trend was evident at the higher angles of attack (Fig. 6): the block-structured and overset results tended to be fairly tightly clustered, whereas the unstructured results showed a larger variation (scatter). Although not shown, results at $\alpha=21^{\circ}$ were similar to those in Fig. 6 , whereas results at $\alpha=22.4^{\circ}$ showed large scatter for all CFD results.

\section{Cases 2 and 3: Configuration with Additional Components}

Including the slat and flap support brackets in the CFD computations had some influence on the forces and pitching moment, as shown in Fig. 7 for medium grids (compare Figs. 7(b), (d), and (f) with Figs. 4(a), (c), and (e)). For example, including the brackets had the general tendency at $R e_{c}=15.1$ million to slightly decrease $C_{L}$ over the 
entire lift curve. Figure 7 also highlights the effects of Reynolds number. Ignoring a few outlier computations, at both Reynolds numbers much of the linear portion of the polar $C_{L}$ was under-predicted, $C_{D}$ was over-predicted, and the magnitude of $C_{M}$ was over-predicted. Collectively, the CFD showed a greater spread near maximum lift, with some under-predicting and some over-predicting $C_{L, \max }$ and the angle at which it occurred. In Fig. 8, the idealized profile drag coefficient $C_{D p}$ is shown. Displaying $C_{D p}$ instead of $C_{D}$ helps to expand the scale of the plot and see the drag variation better.

Only a few participants investigated the effects of including transition. Lift, idealized profile drag, and pitching moment coefficients are shown in Fig. 9. (Note that 003 and 009 used Config 4 whereas 012 used Config 5.) The results were mixed. Recall that the 009.2 results were outliers for non-transitional results, so it is not clear whether the trends displayed here are representative. For 003, including transition had little influence on the lift and idealized drag below $C_{L, \max }$, and tended to only slightly lower the pitching moment magnitude in general. However, for 012 transition had more significant effects. In particular, lift was increased over the entire lift curve. This trend of transition causing higher lift is consistent with conclusions from NASA Trapezoidal Wing investigations. ${ }^{7,18,20,22,28}$

There were only three participants who included computations for Case 3 (the configuration with pressure tube bundles alongside the slat brackets): 012, 021, and 026. Results for $C_{L}$ are shown in Fig. 10. Participant 012 only computed Case 3, so the influence of the pressure tube bundles cannot be isolated. However, both 021 and 026 computed on both Config 4 (Case 2) and Config 5 (Case 3). In Figs. 10(b) and (c) one can see that the pressure tube bundles had little influence except near stall, where they caused loss of lift. This trend was consistent with an experimental investigation. ${ }^{33}$ Although it is not clear which configuration yielded better agreement with experiment, certainly the potential influence of the pressure tube bundles on stall behavior for this model cannot be ignored.

\section{Iterative Convergence}

One of the conclusions from HiLiftPW-1 was that iterative convergence information should be collected from future workshop participants. This collection was done for HiLiftPW-2 for Case 1, although not all participants provided the requested information (see Tables 2 and 3). All who did comply provided force and moment histories; many also included various residual convergence histories. Some representative results are plotted in Figs. 11 and 12 for $\alpha=7^{\circ}$. In Fig. 11, results are shown from those entries for which the final $C_{L}$ still appeared to be changing by the last reported iteration. Iterations are normalized so that 0 represents the first iteration reported and 1 represents the last. Entry 004.1, 005.1, and 005.2 each showed a changing $C_{L}$ with negative slope at the last iteration, while 011.1, 011.3, and 022 each showed significant oscillatory behavior (note that 022 was run time-accurately). As shown in Fig. 11(b), the 004.1, 005.1, and 005.2 entries indicated converging density equation residuals (all residuals have been normalized so that they start at 1). Both 011.1 and 011.3 displayed "stalled" residual behavior, and 022 did not supply residual information.

Figure 12 shows representative results from several entries for which the final $C_{L}$ appeared to be better converged (relatively flat or unchanging). Interestingly, most of the residuals for these apparently better-converged results either "stalled" or leveled off. Three of the representative results drove the residuals down about 5 or 6 orders of magnitude prior to leveling off, while two exhibited weaker convergence over the iteration range reported. Although not shown, representative iterative results for other angles of attack were similar to those shown here. These results indicate that the HiLiftPW-2 high lift cases were generally not easy to converge for many CFD solvers. They also offer a caution when analyzing the workshop data: insufficient iterative convergence may account for some of the differences between different CFD codes.

\section{Velocity Profiles}

Velocity data were collected for Cases 1,2, and 3, although not all participants provided the requested information. In this section, we divide a sampling of the results into two parts. First, results are shown for Case 1 (CFD only no brackets), for which CFD results at $\alpha=7^{\circ}$ are compared to each other for consistency. (Because Case 1 did not involve brackets, experimental data are not included.) Second, results are shown for Cases 2a and 3a, for which CFD results at $\alpha=7^{\circ}$ and $18.5^{\circ}$ are compared to low Reynolds number experiment.

Figures 13 and 14 show CFD Case 1 results at $\alpha=7^{\circ}$ at all eleven stations. As depicted in Fig. 13(a), the stations were labeled in the experiment according to their spanwise row location ( 1 for $y=246.386 \mathrm{~mm}, 2$ for $y=979.596$ $\mathrm{mm}$, and 3 for $y=1223.999 \mathrm{~mm}$ ), then by area (B for near the slat trailing edge, C for forward part of the main wing, $\mathrm{D}$ for aft part of the main wing, and $\mathrm{E}$ for the flap). The final number designation stands for the line number extracted within the area. In the figures, several outliers have been highlighted in blue. In some cases these results may have been different due to errors made by the participants when extracting the velocity profiles. Ignoring the outliers, the 
results in red show reasonably consistent velocity profiles at many of the locations, but this consistency deteriorated at the outboard two stations on the rear part of the main wing and on the flap. The results at location 3E2 showed a very large variation.

Inspecting the plots in Figs. 13 and 14, one can see that some of the results showed distinct, relatively deep wake profiles and some did not. This difference was primarily a function of the grid employed. As an example, Fig. 15 shows results at location 3E1, divided among different grids. Structured results on medium grids A and E are highlighted in bold blue in Fig. 15(a), unstructured results on medium grids $\mathrm{A}$ and $\mathrm{C}$ are highlighted in bold red in Fig. 15(b), and unstructured results on medium grids B and D are highlighted in bold red in Fig. 15(c). The B and D grids yielded significantly smeared (under-resolved) wake profiles from the upstream elements compared to the other grids. Even use of the fine B and D grids (shown in Fig. 15(d)) did not improve this aspect of the results at all. Although not shown, results at other locations showed similar trends.

Figure 16 shows low Reynolds number results at three selected stations and two angles of attack. The CFD results include both Case 2a (brackets included) and Case 3a (brackets and pressure tube bundles included). Aside from a few outliers, the CFD results were reasonably consistent with each other at $\alpha=7^{\circ}$, but less so at $\alpha=18.5^{\circ}$. CFD results tended to be shifted from experiment, predicting velocities too low. Only two participants computed Case 3a; there were no notable consistent trends exhibited at these locations by adding the pressure tube bundles.

\section{E. Surface Pressure and Skin Friction Coefficients}

A significant amount of surface pressure and skin friction data was collected from the participants, along ten different spanwise stations for all cases, as well as at many different angles of attack. Due to the large volume of data collected, much of it has not yet been analyzed. Here, a few results are highlighted.

Figure 17 shows a few typical $C_{p}$ results at $\alpha=7^{\circ}$ at three selected locations. (The particular entries shown here-024.1, 020, and 002.1 — were randomly selected to be representative. There were variations among the different CFD entries as would be expected, with larger variations occurring on the flap as well as at outboard stations.) On the flap at the inboard-most station $2 y / B=0.15$ in Fig. 17(a), high Reynolds number results with no brackets tended to predict too much suction on the upper surface compared to experiment as the grid was refined. When including the brackets (Fig. 17(b)), the opposite occurred and CFD predicted too little upper surface suction at this location. The trend with Reynolds number was not captured. On the slat at the station $2 y / B=0.89$ in Fig. 17(c), little influence of grid was exhibited for high Reynolds number results with no brackets included, and the pressures on the suction side were somewhat low. Including brackets (Fig. 17(d)) helped the CFD to capture the upper surface $C_{p}$ levels; there was also excellent agreement with Reynolds number trends. In Fig. 17(e), results are shown for the flap at the outboardmost span station $2 y / B=0.96$ at high Reynolds number with no brackets. CFD missed the pressures on the lower surface. Including brackets (Fig. 17(f)) helped the CFD to capture the lower surface levels accurately. The Reynolds number trend was qualitatively captured, but the upper surface pressure levels did not match experiment.

Figure 18 shows Case $2 \mathrm{a}$ and $2 \mathrm{~b} C_{p}$ results at two span stations at $\alpha=20^{\circ}$ near $C_{L, \max }$. Here, results from all participants who submitted these cases are included with the exceptions of 007.1 (because results were very poor and probably extracted incorrectly) and 015.1/015.2 (because they did not include brackets). Low Reynolds number CFD results are represented by solid red lines or (for data that were not ordered) by tiny red symbols. High Reynolds number CFD results are represented by blue lines or tiny blue symbols. The experimental data are represented by large symbols. Near mid-span at $2 y / B=0.54$, CFD results on the slat and main elements were very consistent, and agreed well with experiment (both levels and Reynolds number trends). On the flap, however, a larger degree of variation was exhibited among the CFD solutions. Qualitatively, a trend with Reynolds number was seen, but the experimental levels were not captured. At the span station $2 y / B=0.89$, CFD results exhibited significant variation on all three elements. Some trends with Reynolds number were seen, but pressure levels were particularly missed on the flap at low Reynolds number.

Surface skin friction coefficients were optional for the participants of the workshop, so less data were provided. Also, no experimental skin friction data were available for comparison. Figure 19 shows Case $2 \mathrm{a}$ and $2 \mathrm{~b} C_{f, x}$ CFD results at two different angles of attack on the flap at span stations $2 y / B=0.15,0.54$, and 0.89 . (Note that 009.1 results were left out due to obvious errors in $C_{f}$ extraction.) Results at $\alpha=7^{\circ}$ are in the left column, and results at $\alpha=16^{\circ}$ are on the right. Again, red lines and symbols represent low Reynolds number, and blue lines and symbols represent high Reynolds number. The CFD results were generally very close to each other on the lower surface, but exhibited more variation on the upper surface. At both angles of attack, some of the CFD results showed a small amount of trailing edge separation at $2 y / B=0.54$. At $2 y / B=0.89$, more separation was evident, particularly at the low Reynolds number condition. 


\section{F. Statistical Analysis}

In the statistical analysis of this section, the basic method used by Morrison ${ }^{49}$ was employed. For any quantity of interest ( $C_{L}$ for example), the scatter limits were established as $\hat{\mu} \pm K \hat{\sigma}$, where $\hat{\mu}$ is the median of the sorted data, $\hat{\sigma}$ is the standard deviation, and $K$ is a confidence interval coverage factor, taken to be $K=\sqrt{3}$. The coefficient of variation, defined as $C_{v}=\hat{\sigma} / \hat{\mu}$, provides a measure to compare the variation of populations with different medians. This same statistical methodology was also used in the analysis of HiLiftPW-1. ${ }^{5}$ A statistical outlier (a point residing outside of the scatter limits) is by definition different from the results that lie within the scatter limits. In terms of CFD solutions, being an outlier means that some aspect of the CFD solution was significantly different from the collective, and its cause(s) should be investigated. In the plots to follow, any sample lying well outside of the limits is noted in the figure. However, because the scatter limits depend on the choice of $K$, the fact that a particular point lies outside may or may not be significant.

Figure 20 shows results from the statistical analysis of Case 1 (no brackets, high $R e$ ) at $\alpha=7^{\circ}$. The top row is $C_{L}$, middle row is $C_{D}$, and bottom row is $C_{M}$. The left column shows coarse grid results, middle column is medium grid results, and right column is fine grid results. In all cases, (as is desirable) the scatter range and $C_{v}$ both decreased as grid was refined. However, the biggest difference occurred between coarse and medium grids, and very little change occurred between the medium and fine grids. The few outliers produced lift values that were too low compared to the collective. Median lift increased with grid refinement while pitching moment decreased. Drag changed very little.

Figure 21 shows results from the statistical analysis of Case 1 at $\alpha=16^{\circ}$. In this case, scatter range and $C_{v}$ again decreased for $C_{L}$ with grid refinement, but for drag they remained fixed on the medium and fine grids and for pitching moment the scatter range increased slightly on the fine grid. The outliers again produced lift values that were too low compared to the collective. Median lift increased with grid refinement while pitching moment decreased and drag increased only slightly.

Figure 22 summarizes the coefficients of variation for Case 1 at $\alpha=7^{\circ}$ and $16^{\circ}$ and compares them with the $C_{v}$ values from HiLiftPW-1. $C_{v}$ levels for lift and drag were somewhat similar between the two workshops, although the current HiLiftPW-2 results decreased less (flattened more) between the medium and fine grids (i.e., the fine grid $C_{v}$ levels for HiLiftPW-2 tended to be higher than the fine grid $C_{v}$ levels for HiLiftPW-1). $\left|C_{v}\right|$ levels for pitching moment were higher than for HiLiftPW-1, and also flattened more than for HiLiftPW-1 between the medium and fine grids. In other words, for HiLiftPW-2, going from medium to fine grids overall did not tend to bring the various CFD results much closer together, as would generally be expected. The reason for this is not known.

In Fig. 23, an attempt was made to seek trends for Case 1 by turbulence model, by grid type, and by grid. Specific trends should manifest themselves by showing a particular bias compared to the median. However, few significant trends stood out among the results with a sufficiently large population; most symbols appeared to be fairly randomly distributed. One possible trend was that structured-overset results (corresponding to grid E) for $\alpha=16^{\circ}$ tended to give higher than average lift. Another noteworthy point is that at both angles of attack the two SA results giving the lowest lift were 007.2 and 011.1. Recalling the verification exercise from Fig. 3(b), these particular implementations of SA were suspect.

Figures 24 through 28 display statistical analyses of forces and pitching moments for Cases $2 \mathrm{a}$ and $\mathrm{b}$ (which included brackets) at $\alpha=0^{\circ}, 7^{\circ}, 16^{\circ}, 20^{\circ}$, and $21^{\circ}$, respectively. Experimental values (yellow circles) are also displayed in the figures for comparison. For both Reynolds numbers at $\alpha=0^{\circ}$ and $7^{\circ}$, the median values of lift, drag, and pitching moment were somewhat different compared to experiment, but the CFD scatter bands contained the experiment. At $\alpha=16^{\circ}$, the median CFD value of $C_{L}$ agreed almost perfectly with experiment at both Reynolds numbers, but drag was significantly over-predicted and pitching moment was significantly under-predicted. At $\alpha=$ $20^{\circ}$, the median CFD value of $C_{L}$ was again excellent compared with experiment at both Reynolds numbers. Drag was predicted well at the low Reynolds number condition, but again over-predicted at high Reynolds number. Moment was significantly under-predicted at both Reynolds numbers. At $\alpha=21^{\circ}$, the median CFD value of $C_{L}$ was only slightly high compared to experiment (the CFD scatter band contained the experiment). Drag was under-predicted at the low Reynolds number condition and over-predicted at high Reynolds number. Moment was again significantly under-predicted.

Referring back to Fig. 7, recall that the entries exhibited more spread near stall (with some entries over-predicting $C_{L, \max }$ and some under-predicting it). Interestingly, the median value from the CFD codes happened to come out relatively accurately in predicting the lift at the highest angles of attack. However, this may have been luck. At high Reynolds number the median $C_{L}$ of the CFD was still increasing at $\alpha=21^{\circ}$, whereas in the experiment it was starting to decrease. Furthermore, the large spread of CFD behavior near $C_{L, \max }$ makes it doubtful that the CFD codes collectively and consistently captured the physical mechanism(s) responsible for stall. The reasons for the mis-predictions in drag and pitching moment over much of the polars above $\alpha=7^{\circ}$ is not known. 
To further explore how well the median values from the CFD codes captured Reynolds number trends, delta values of $C_{L}, C_{D}$, and $C_{M}$ are plotted in Fig. 29. For example, $\Delta C_{L}=C_{L, h i R e}-C_{L, l o R e}$. Overall, the shapes of the delta curves as a function of angle of attack were qualitatively captured, but $\Delta C_{L}$ at low $\alpha$ was over-predicted, and the magnitude of the negative $\Delta C_{D}$ at high $\alpha$ was under-predicted.

\section{Conclusions}

The entries from the second AIAA High-Lift Prediction Workshop (HiLiftPW-2) have been summarized, both by looking at collective results compared with experiment and through statistical analyses. A large number of surface pressures, skin frictions, and velocity profiles were collected, but only a small portion of these have been analyzed to date, and only a few representative plots have been shown here.

An optional turbulence model verification case was included in this workshop, and computed by a few of the participants. For those who used the SA model, there was near-perfect consistency among three entries, one other entry was very close, and two exhibited notable differences. These two differences suggest potential problems or inconsistencies in implementation, which should be investigated by the owners of the codes in question.

For the DLR-F11 case in general, the importance of including slat and flap brackets when comparing with experiment was established. From previous experimental oil flow, it appeared that two slat tracks were influential in causing large wedge-shaped regions of separated flow on the main element near stall. Without brackets, the CFD could not possibly capture this type of stall mechanism, and indeed most entries without brackets tended to predict increasing $C_{L}$ well past the nominal stall angle. There was also some evidence from the few participants who ran Case 3 that pressure tube bundles included alongside the slat tracks on the wind tunnel model had an influence on the flow field near $C_{L, \max }$.

In terms of consistency, the CFD exhibited spreads in results that tended to remain about the same when going from the medium to the fine grid levels. In other words, the CFD scatter did not decrease much past a certain grid refinement level; the reasons for this are not known. Scatter was larger at the angles of attack near stall, as expected. A small part of the CFD inconsistencies may have been due to poor or insufficient iterative convergence, as participants had some difficulty fully converging many of the cases. Looking at details such as pressure coefficients and velocity profiles, the inconsistencies between CFD results tended to be larger on the flap as well as at the outboard stations of the wing. Some of the unstructured grids were shown to be poor for capturing the wakes of upstream elements.

Most of the participants used the Spalart-Allmaras turbulence model or variant. Nonetheless, the variation among entries using this model appeared to be as great as differences due to the use of other turbulence models. In other words, no clear trends with turbulence modeling were exhibited in the results. A few participants investigated the use of transition, but unlike results for the NASA Trapezoidal Wing of the first high lift workshop, results were mixed and no clear trends stood out.

When including the slat and flap brackets, the median CFD results tended to be slightly low predicting lift at $\alpha=7^{\circ}$, accurate at $\alpha=16^{\circ}$ and $20^{\circ}$, then somewhat high at $\alpha=21^{\circ}$. However, the scatter range for CFD's $C_{L}$ results (approximately 0.2 at low angles of attack and typically higher in the range of $0.3-0.6$ at high angles of attack) always included the experimental values. This was generally not the case for drag and pitching moment, both of which were consistently mis-predicted at angles of attack above $\alpha=7^{\circ}$. Reynolds number trends were only qualitatively captured by the CFD.

HiLiftPW-2 continued in the tradition of the first workshop in the series, by again bringing together many researchers from around the world with a variety of CFD codes to analyze a specific high lift configuration. This new configuration offered different challenges from the configuration of the first workshop. The CFD results again lacked consistency to the levels desired, but much was learned by quantifying the differences and by taking steps to attempt to isolate possible causes. For example, including verification testing as well as iterative convergence information were both very useful aspects that could serve as prerequisites for future workshops. Workshops like this are very valuable for the aeronautical engineering community, and should continue to be pursued.

\section{Acknowledgments}

The authors wish to express their gratitude to HiLiftPW-2 committee members Dave Levy and Mark Chaffin of Cessna for their help preparing plots and grid analysis for the workshop talks. Thanks also go to Stefan Melber of DLR, Leonel Serrano, Raul Mendoza, Karuna Rajagopa, Neal Harrison, Yoram Yadlin, and John Vassberg of Boeing, and committee members Carolyn Woeber of Pointwise, Mike Long of University of Wyoming, Mark Chaffin of Cessna, and Tony Sclafani of Boeing for creating the grids. The authors further acknowledge committee members 
Ralf Rudnik and Kerstin Huber of DLR for pulling together and organizing the DLR-F11 data. Finally, the authors thank all committee members mentioned above plus Thomas Wayman of Gulfstream, Tom Pulliam of NASA Ames, Dimitri Mavriplis of University of Wyoming, and Judi Hannon of NASA Langley for their continued support with the planning and execution of the high lift workshops and special sessions.

\section{References}

${ }^{1}$ Rumsey, C. L. and Ying, S. X., "Prediction of High Lift: Review of Present CFD Capability," Progress in Aerospace Sciences, Vol. 38, 2002, pp. $145-180$

${ }^{2}$ Johnson, P. L., Jones, K. M., and Madson, M. D., "Experimental Investigation of a Simplified 3D High Lift Configuration in Support of CFD Validation," AIAA Paper 2000-4217, August 2000.

${ }^{3}$ Hannon, J. A., Washburn, A. E., Jenkins, L. N., and Watson, R. D., "Trapezoidal Wing Experimental Repeatability and Velocity Profiles in the 14- by 22-Foot Subsonic Tunnel (Invited)," AIAA Paper 2012-0706, January 2012.

${ }^{4}$ Slotnick, J. P., Hannon, J. A., and Chaffin, M., "Overview of the First AIAA CFD High Lift Prediction Workshop (Invited)," AIAA Paper 2011-862, January 2011.

${ }^{5}$ Rumsey, C. L., Slotnick, J. P., Long, M., Stuever, R. A., and Wayman, T. R., "Summary of the First AIAA CFD High-Lift Prediction Workshop," Journal of Aircraft, Vol. 48, No. 6, 2011, pp. 2068-2079.

${ }^{6}$ Long, M. and Mavriplis, D., "NSU3D Results for the First AIAA High Lift Prediction Workshop,” AIAA Paper 2011-863, January 2011.

${ }^{7}$ Steed, R. G. F., "High Lift CFD Simulations with an SST-Based Predictive Laminar to Turbulent Transition Model," AIAA Paper 2011-864, January 2011.

${ }^{8}$ Wiart, L. and Meunier, M., "Computational Assessment of the HiLiftPW-1 Trap-Wing Model Using the elsA CFD Software," AIAA Paper 2011-865, January 2011.

${ }^{9}$ Sclafani, A. J., Slotnick, J. P., Vassberg, J. C., Pulliam, T. H., and Lee, H. C., "OVERFLOW Analysis of the NASA Trap Wing Model from the First High Lift Prediction Workshop," AIAA Paper 2011-866, January 2011.

${ }^{10}$ Eliasson, P., Peng, S.-H., and Hanifi, A., "Improving the Prediction for the NASA High-Lift Trap Wing Model," AIAA Paper 2011-867, January 2011.

${ }^{11}$ Reyes, D. A., Girimaji, S. S., Pandya, M., and Abdol-Hamid, K. S., "Computations of High-Lift Wing-Body Configuration on Unstructured Grids using $k-\omega$ Models," AIAA Paper 2011-868, January 2011.

${ }^{12}$ Fares, E. and Nolting, S., "Unsteady Flow Simulation of a High-Lift configuration using a Lattice Boltzmann Approach," AIAA Paper 2011-869, January 2011.

${ }^{13}$ Park, M. A., Lee-Rausch, E. M., and Rumsey, C. L., "FUN3D and CFL3D Computations for the First High Lift Prediction Workshop," AIAA Paper 2011-936, January 2011.

${ }^{14}$ Murayama, M., Yamamoto, K., and Tanaka, K., "CFD Comparison Study for Trapezoidal High-Lift Wing Configurations by Structured and Unstructured Mesh Method," AIAA Paper 2011-937, January 2011.

${ }^{15}$ Crippa, S., Melber-Wilkending, S., and Rudnick, R., "DLR Contribution to the First High Lift Prediction Workshop," AIAA Paper 2011-938, January 2011.

${ }^{16}$ Antunes, A. P., Azevedo, J. L. F., and da Silva, R. G., "Numerical Simulations of Turbulent Flow over a High-Lift Configuration," AIAA Paper 2011-3006, June 2011.

${ }^{17}$ Pandya, M. J., Abdol-Hamid, K. S., and Parlette, E. B., "CFD Computations for a Generic High-Lift Configuration Using TetrUSS," AIAA Paper 2011-3008, June 2011

${ }^{18}$ Eliasson, P., Hanifi, A., and Peng, S.-H., "Influence of Transition on High-Lift Prediction for the NASA Trap Wing Model," AIAA Paper 2011-3009, June 2011.

${ }^{19}$ Lee, H. C. and Pulliam, T. H., "Effect of Using Near and Off-body Grids with Grid Adaption to Simulate Airplane Geometries," AIAA Paper 2011-3985, June 2011.

${ }^{20}$ Rumsey, C. L. and Lee-Rausch, E. M., "NASA Trapezoidal Wing Computations Including Transition and Advanced Turbulence Modeling," AIAA Paper 2012-2843, June 2012.

${ }^{21}$ Murayama, M., Ito, Y., Tanaka, K., and Yamamoto, K., "Computational Studies of the NASA High-Lift Trap Wing Using Structured and Unstructured Grid Solvers," AIAA Paper 2012-2844, June 2012.

${ }^{22}$ Crippa, S. and Krimmelbein, N., "Transitional Flow Computations of the NASA Trapezoidal Wing with the DLR TAU Code," AIAA Paper 2012-2845, June 2012.

${ }^{23}$ Antunes, A. P., da Silva, R. G., and Azevedo, J. L., "Influence of High-Lift Supporting Systems on the Trapezoidal Wing Aerodynamic Coefficients," AIAA Paper 2012-2846, June 2012.

${ }^{24}$ Cavallo, P. A. and Feldman, G. M., "Quantification of Grid Refinement Effects for NASA High Lift Trap Wing Using Error Transport Model," AIAA Paper 2012-2847, June 2012.

${ }^{25}$ Moitra, A., Gujar, P., and Gupta, P., "Investigation of Solution Methodology for Resolution of Side of Body Flow Separation," AIAA Paper 2012-2848, June 2012.

${ }^{26}$ Ravindra, K., Shende, N. V., Patel, S. K., and Balakrishnan, N., "Unstructured adaptive mesh calculations for NASA TRAP WING using the code HiFUN," AIAA Paper 2012-2849, June 2012.

${ }^{27}$ Wiart, L. and Moens, F., "Numerical Simulation of the NASA High-Lift Trap Wing with the elsA CFD Software," AIAA Paper 2012-2918, June 2012.

${ }^{28}$ Sclafani, A. J., Slotnick, J. P., Vassberg, J. C., and Pulliam, T. H., "Extended OVERFLOW Analysis of the NASA Trap Wing Wind Tunnel Model," AIAA Paper 2012-2919, June 2012

${ }^{29}$ Shankara, P. and Snyder, D., "Numerical Simulation of High Lift Trap Wing using STAR-CCM+," AIAA Paper 2012-2920, June 2012. 
${ }^{30}$ Lopez, O. D., Ochoa, N., Mahecha, J. C., Leguizamon, S., Escobar, J. A., Ramirez, S., Jimenez, R. A., Giraldo, A. M., and Silva, C., "Numerical Simulation of NASA Trap-Wing Model as a Colombian Contribution to the High-Lift Prediction Workshop," AIAA Paper 2012-2921, June 2012.

${ }^{31}$ Fabiano, E., Fares, E., and Nolting, S., "Unsteady Flow Simulation of High-Lift stall Hysteresis using a Lattice Boltzmann Approach," AIAA Paper 2012-2922, June 2012.

${ }^{32}$ Holman, D. M., Brionnaud, R. M., Martinez, F. J., and Mier-Torrecilla, M., "Advanced Aerodynamic Analysis of the NASA High-Lift Trap Wing with a Moving Flap Configuration," AIAA Paper 2012-2923, June 2012.

${ }^{33}$ Rudnik, R., Huber, K., and Melber-Wilkending, S., "EUROLIFT Test Case Description for the 2nd High Lift Prediction Workshop," AIAA Paper 2012-2924, June 2012.

${ }^{34}$ Hansen, H., Thiede, P., Rudnik, R., Moens, F., and Quest, J., "Overview about the European High Lift Research Programme EUROLIFT," AIAA Paper 2004-0767, January 2004.

${ }^{35}$ Spalart, P. R. and Allmaras, S. R., “A One-Equation Turbulence Model for Aerodynamic Flows,” Recherche Aerospatiale, No. 1, 1994, pp. $5-21$

${ }^{36}$ Shur, M. L., Strelets, M. K., Travin, A. K., and Spalart, P. R., "Turbulence Modeling in Rotating and Curved Channels: Assessing the Spalart-Shur Correction," AIAA Journal, Vol. 38, No. 5, 2000, pp. 784-792.

${ }^{37}$ Dacles-Mariani, J., Zilliac, G. G., Chow, J. S., and Bradshaw, P., "Numerical/Experimental Study of a Wingtip Vortex in the Near Field," AIAA Journal, Vol. 33, No. 9, 1995, pp. 1561-1568.

${ }^{38}$ Edwards, J. R. and Chandra, S., "Comparison of Eddy Viscosity-Transport Turbulence Models for Three-Dimensional, Shock-Separated Flowfields," AIAA Journal, Vol. 34, No. 4, 1996, pp. 756-763.

${ }^{39}$ Rung, T., Bunge, U., Schatz, M., and Thiele, F., "Restatement of the Spalart-Allmaras Eddy-Viscosity Model in Strain-Adaptive Formulation," AIAA Journal, Vol. 41, No. 7, 2003, pp. 1396-1399.

${ }^{40}$ Spalart, P. R., "Strategies for Turbulence Modelling and Simulations," Int. Journal of Heat and Fluid Flow, Vol. 21, 2000, pp. 252-263.

${ }^{41}$ Menter, F. R., “Two-Equation Eddy-Viscosity Turbulence Models for Engineering Applications," AIAA Journal, Vol. 32, No. 8, 1994, pp. 1598-1605.

${ }^{42}$ Menter, F. R., "Improved Two-Equation k-omega Turbulence Models for Aerodynamic Flows," NASA TM 103975 , October 1992.

${ }^{43}$ Goldberg, U., Peroomian, O., Batten, P., and Chakravarthy, S., “The k-e-Rt Turbulence Closure," Engineering Applications of Fluid Mechanics, Vol. 3, No. 2, 2009, pp. 175-183.

${ }^{44}$ Hellsten, A., "New Advanced k-omega Turbulence Model for High-Lift Aerodynamics," AIAA Journal, Vol. 43, No. 9, 2005 , pp. $1857-1869$.

${ }^{45}$ Wilcox, D. C., Turbulence Modeling for CFD, 1st edition, DCW Industries, Inc., La Canada CA, 1988.

${ }^{46}$ Coder, J. G. and Maughmer, M. D., "A CFD-Compatible Transition Model Using an Amplification Factor Transport Equation," AIAA Paper 2013-0253, January 2013.

${ }^{47}$ Langtry, R. B. and Menter, F. R., "Correlation-Based Transition Modeling for Unstructured Parallelized Computational Fluid Dynamics Codes," AIAA Journal, Vol. 47, No. 12, 2009, pp. 2894-2906.

${ }^{48}$ Levy, D., Laflin, K., Tinoco, E., Vassberg, J., Mani, M., Rider, B., Rumsey, C., Wahls, R., Morrison, J., Broderson, O., Crippa, S., Mavriplis, D., and Murayama, M., "Summary of Data from the Fifth AIAA CFD Drag Prediction Workshop," AIAA Paper 2013-0046, January 2013.

${ }^{49}$ Morrison, J. H., "Statistical Analysis of CFD Solutions from the Fourth AIAA Drag Prediction Workshop," AIAA Paper 2010-4673, JuneJuly 2010. 


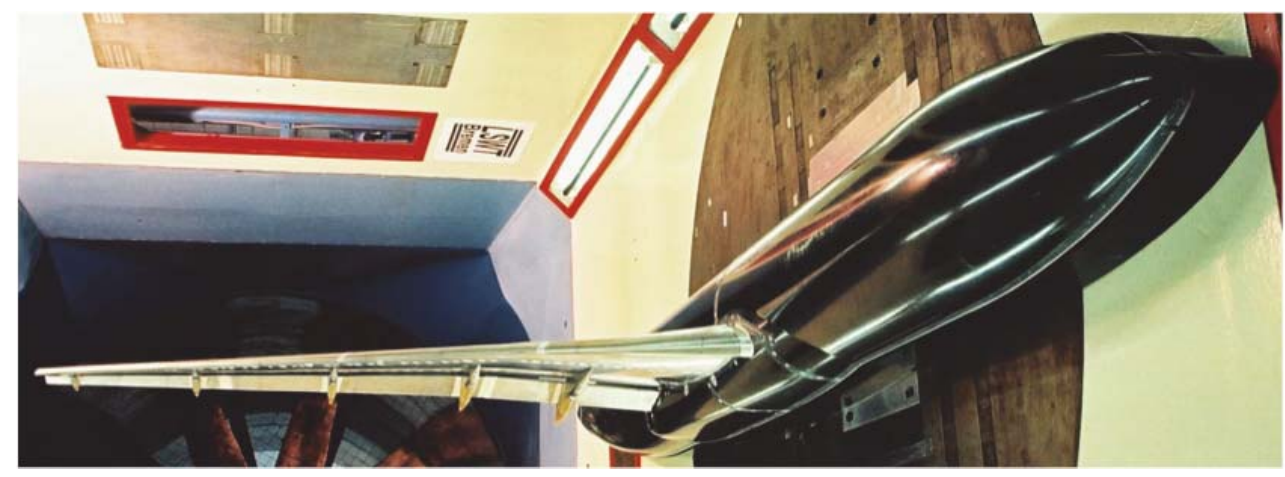

Figure 1. Photograph of DLR-F11 high lift model in the B-LWST wind tunnel.

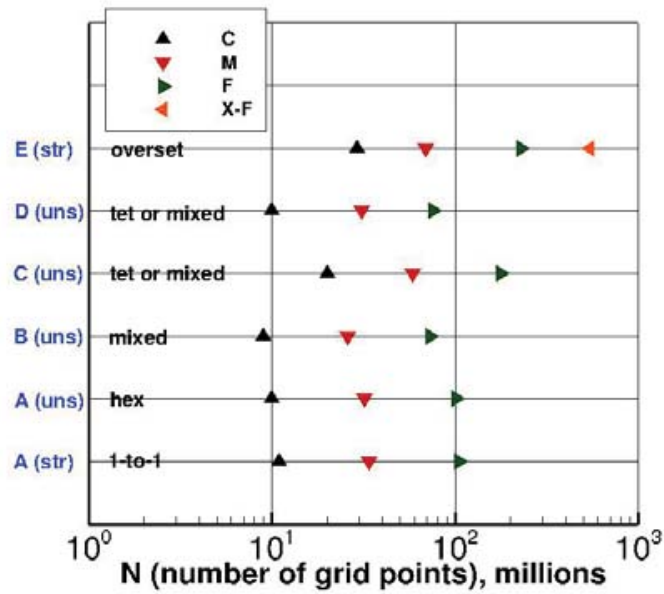

Figure 2. Case 1 supplied grid sizes.

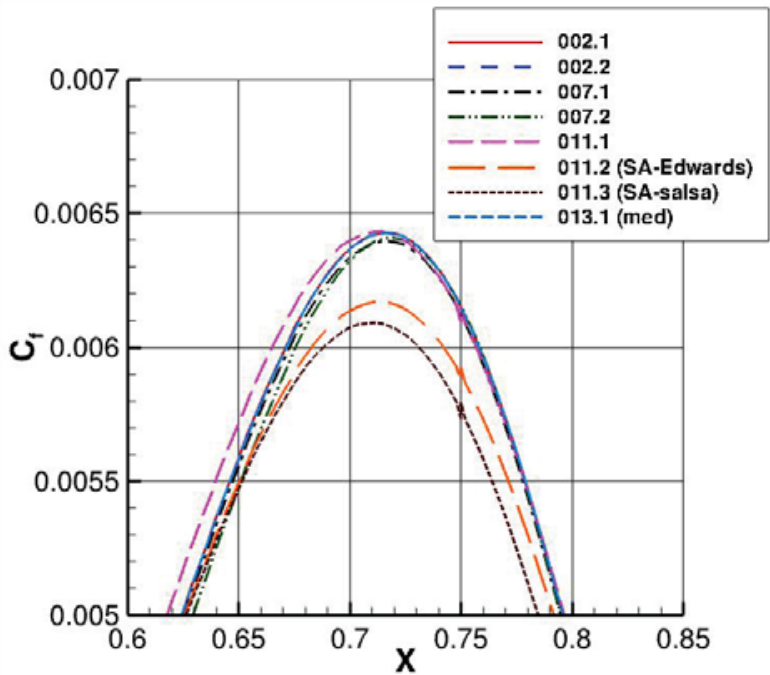

(a) $C_{f}$ on finest grid (detail)

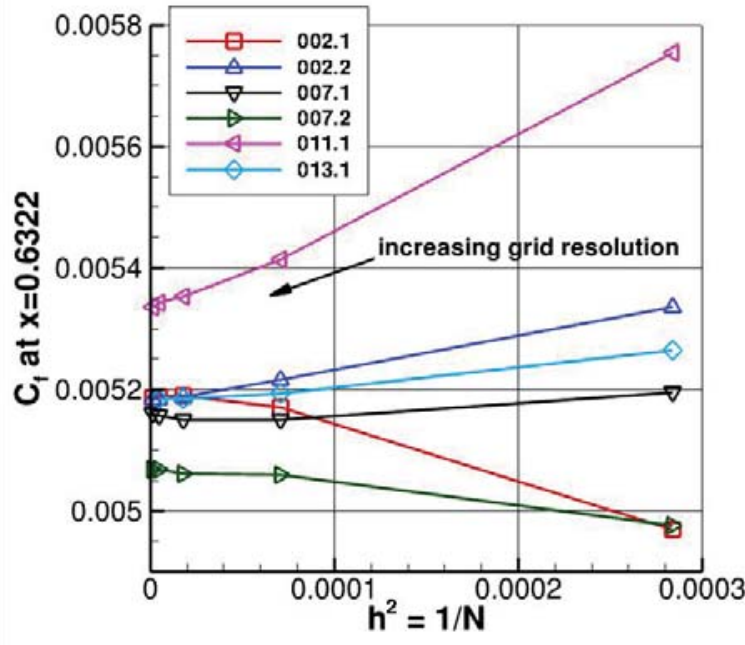

(b) Grid convergence study of $C_{f}$ at $x=0.6322$ (SA only)

Figure 3. 2-D bump verification case using SA model and variants. 


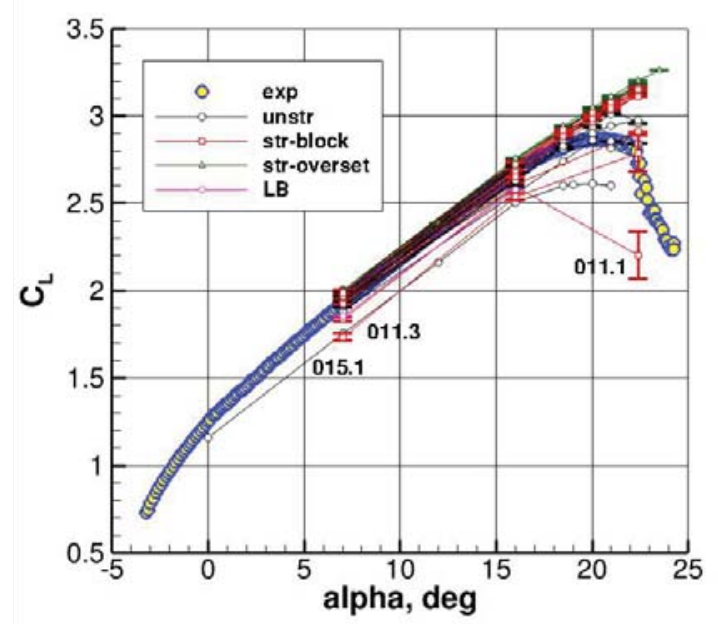

(a) $C_{L}$, medium grids

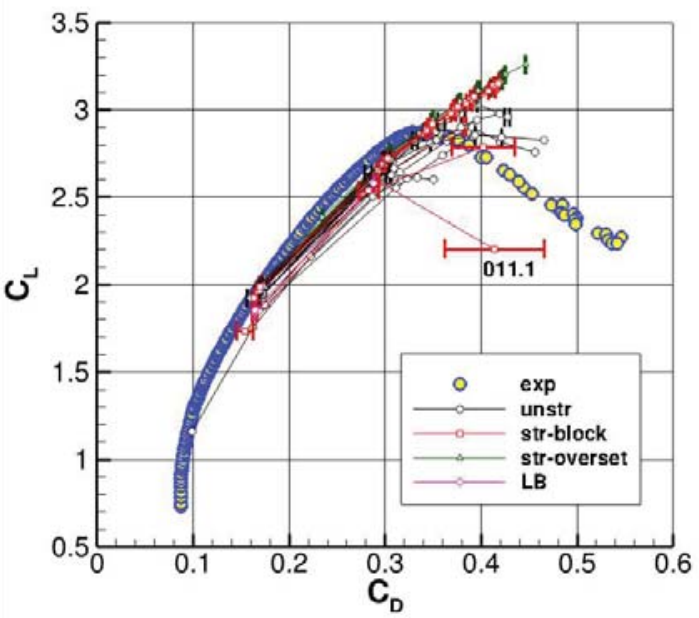

(c) $C_{D}$, medium grids

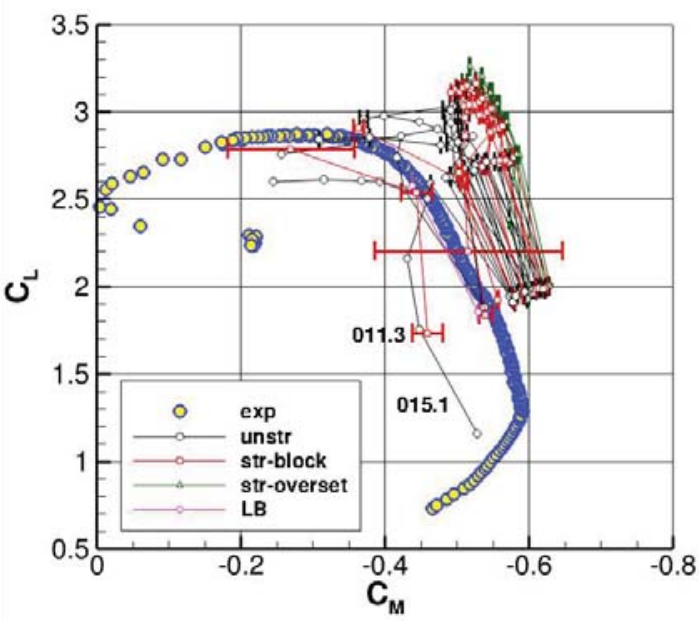

(e) $C_{M}$, medium grids

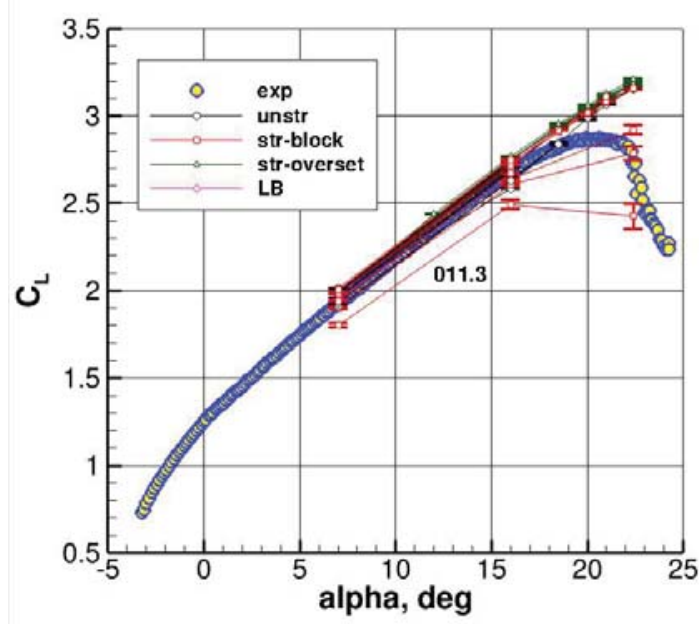

(b) $C_{L}$, fine grids

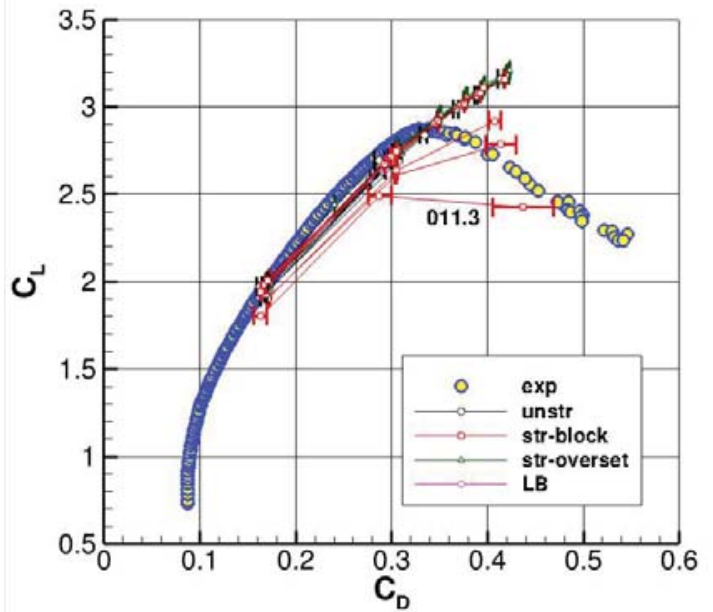

(d) $C_{D}$, fine grids

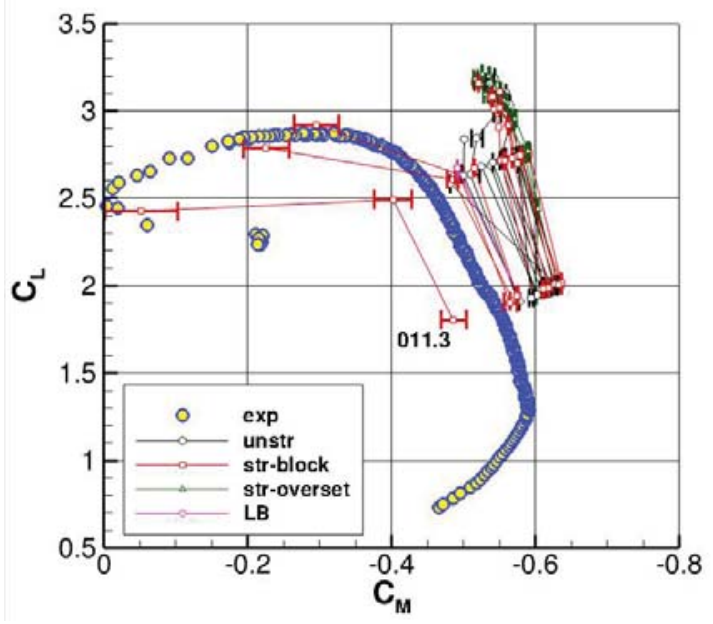

(f) $C_{M}$, fine grids

Figure 4. Case 1 (no brackets, high $R e$ ) forces and pitching moments (experiment shown for reference). 


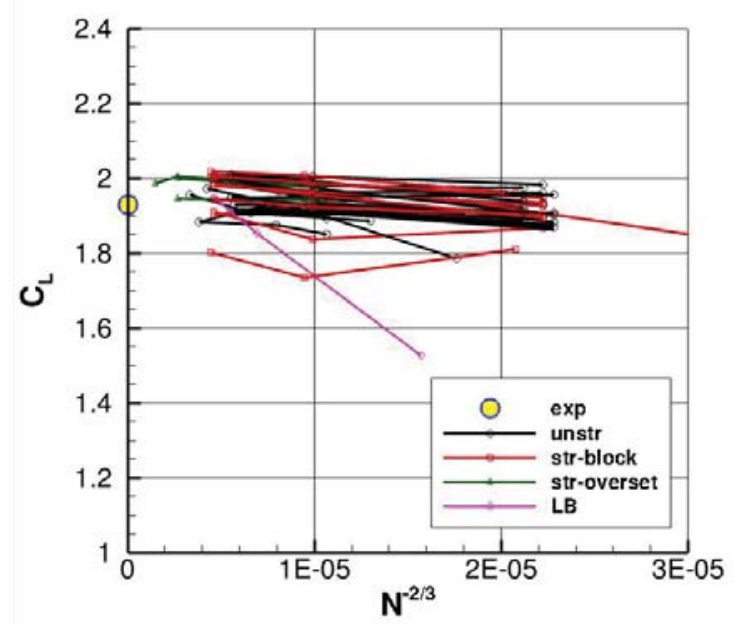

(a) $C_{L}, \alpha=7^{\circ}$

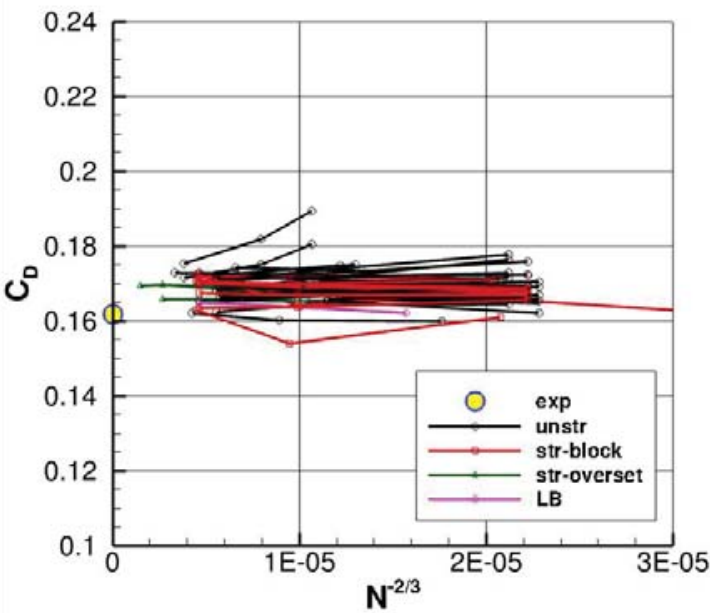

(c) $C_{D}, \alpha=7^{\circ}$

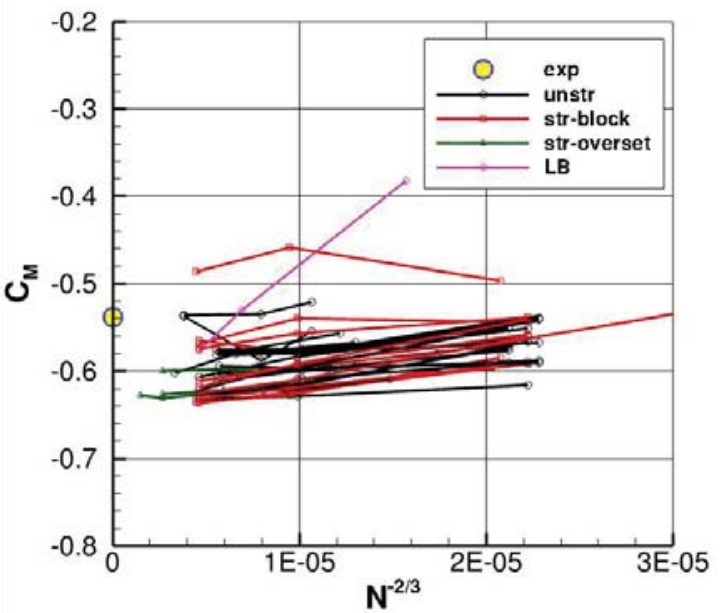

(e) $C_{M}, \alpha=7^{\circ}$

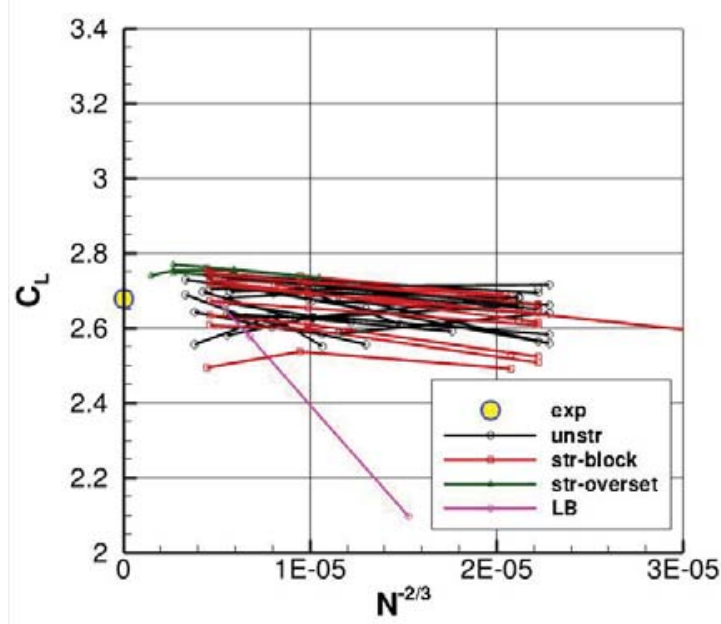

(b) $C_{L}, \alpha=16^{\circ}$

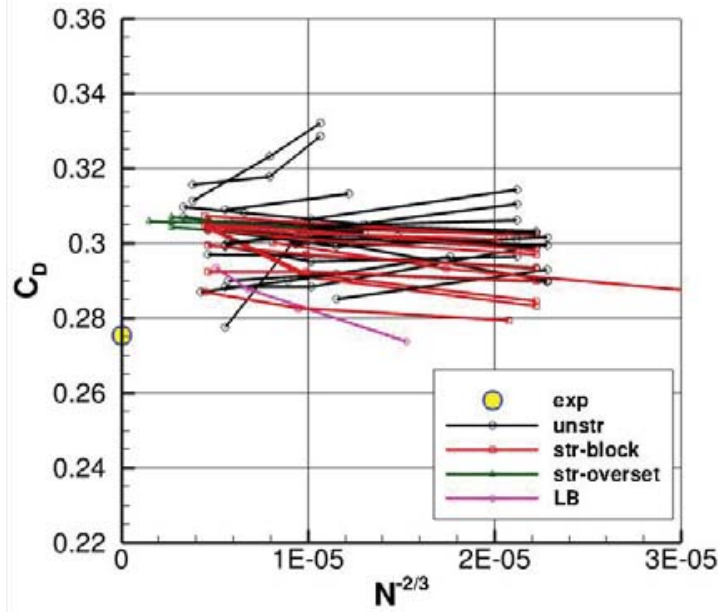

(d) $C_{D}, \alpha=16^{\circ}$

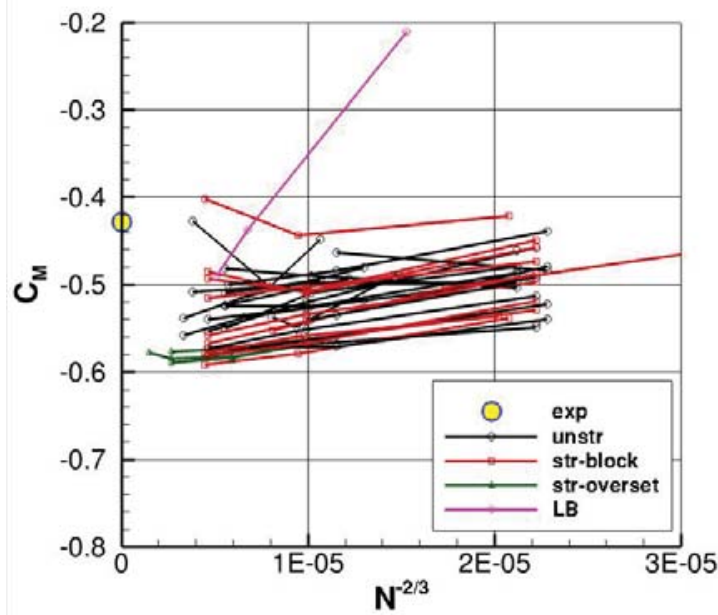

(f) $C_{M}, \alpha=16^{\circ}$

Figure 5. Case 1 (no brackets, high $R e$ ) grid convergence behavior at $\alpha=7^{\circ}$ and $16^{\circ}$ (experiment shown for reference). 


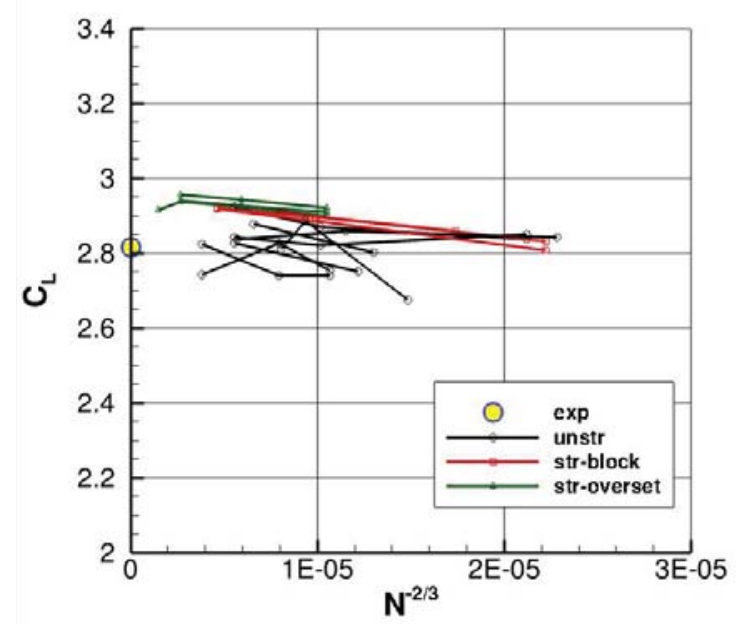

(a) $C_{L}, \alpha=18.5^{\circ}$

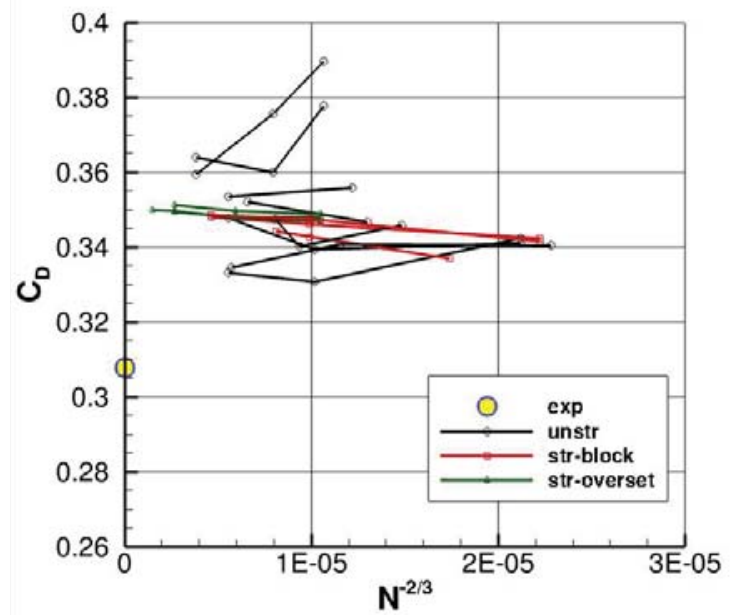

(c) $C_{D}, \alpha=18.5^{\circ}$

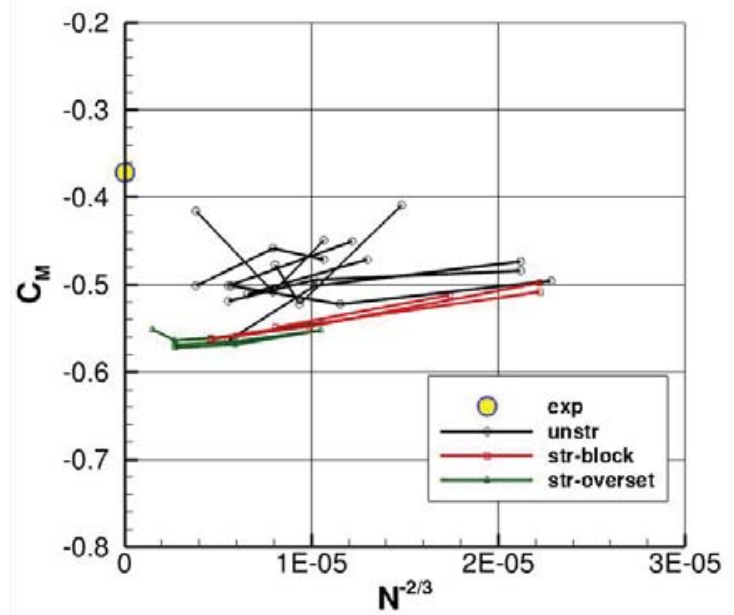

(e) $C_{M}, \alpha=18.5^{\circ}$

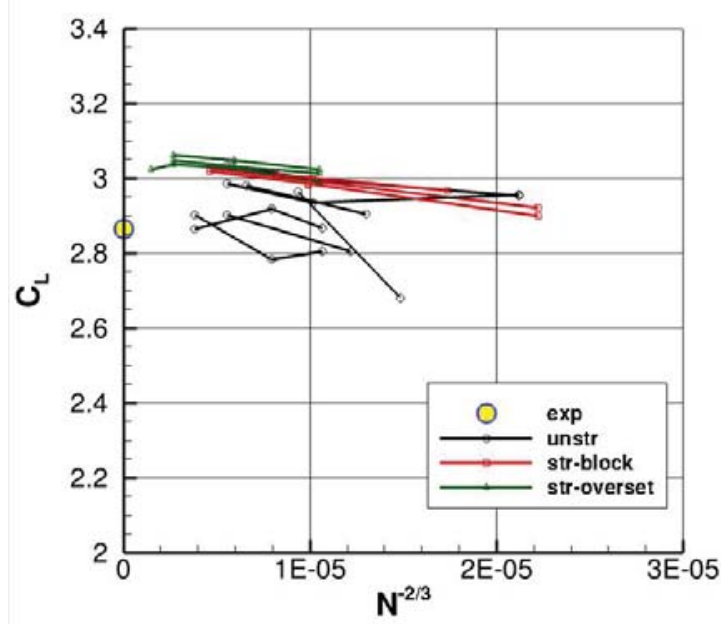

(b) $C_{L}, \alpha=20^{\circ}$

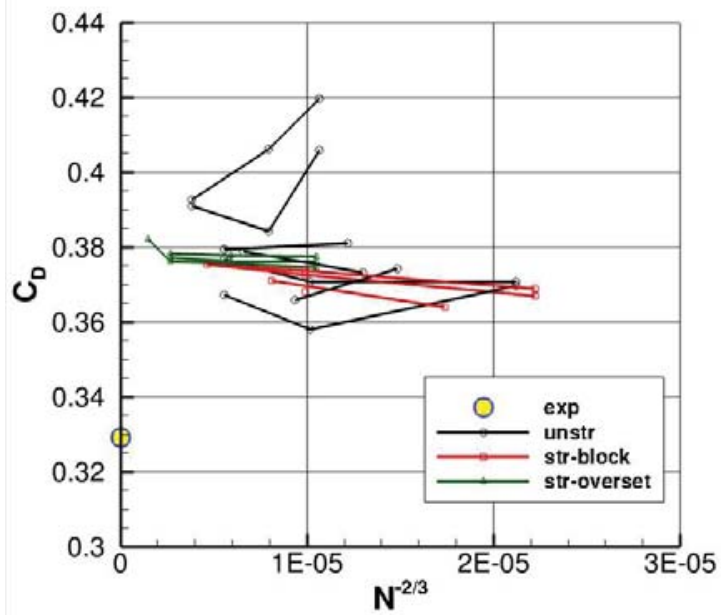

(d) $C_{D}, \alpha=20^{\circ}$

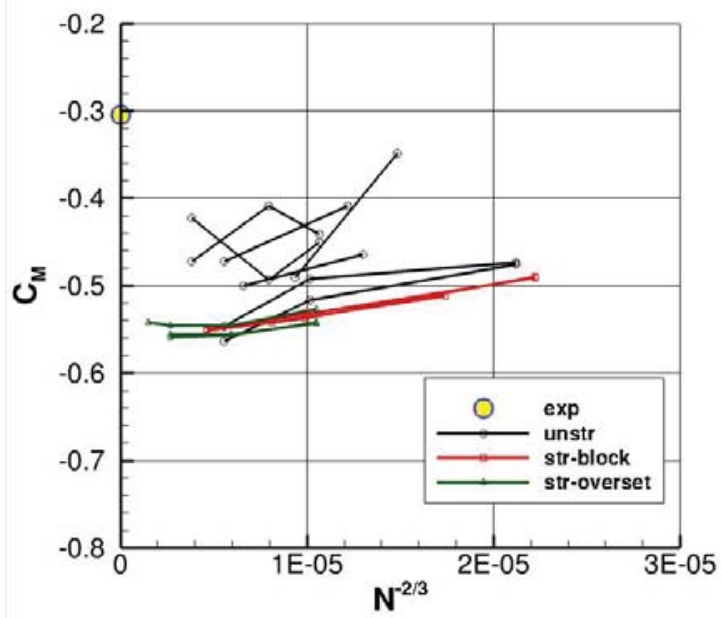

(f) $C_{M}, \alpha=20^{\circ}$

Figure 6. Case 1 (no brackets, high $R e$ ) grid convergence behavior at $\alpha=18.5^{\circ}$ and $20^{\circ}$ (experiment shown for reference). 


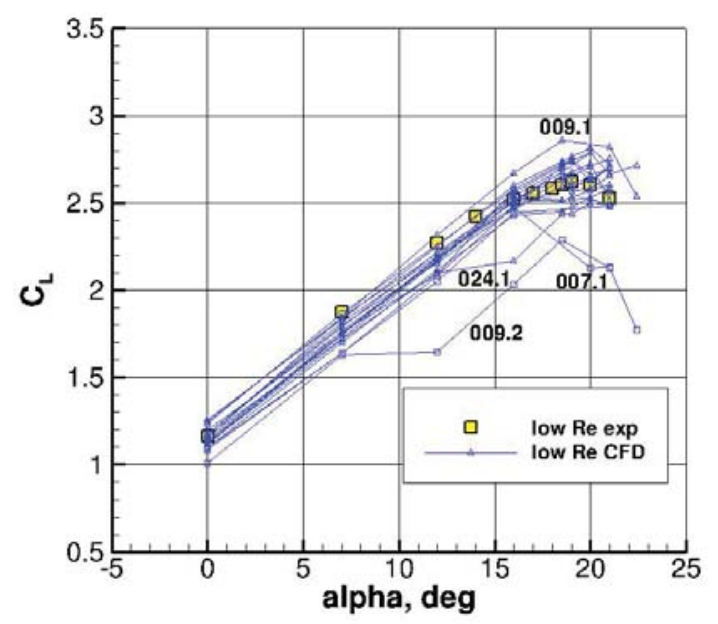

(a) $C_{L}, R e_{c}=1.35 \times 10^{6}$

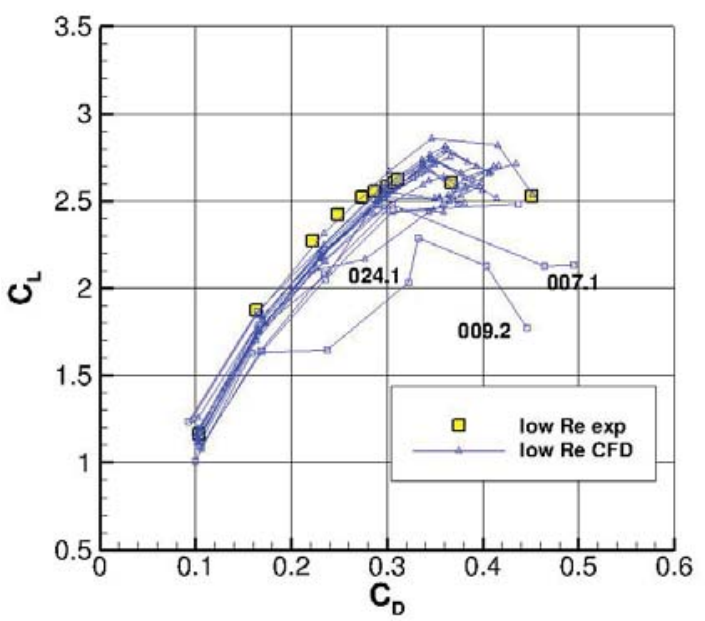

(c) $C_{D}, R e_{c}=1.35 \times 10^{6}$

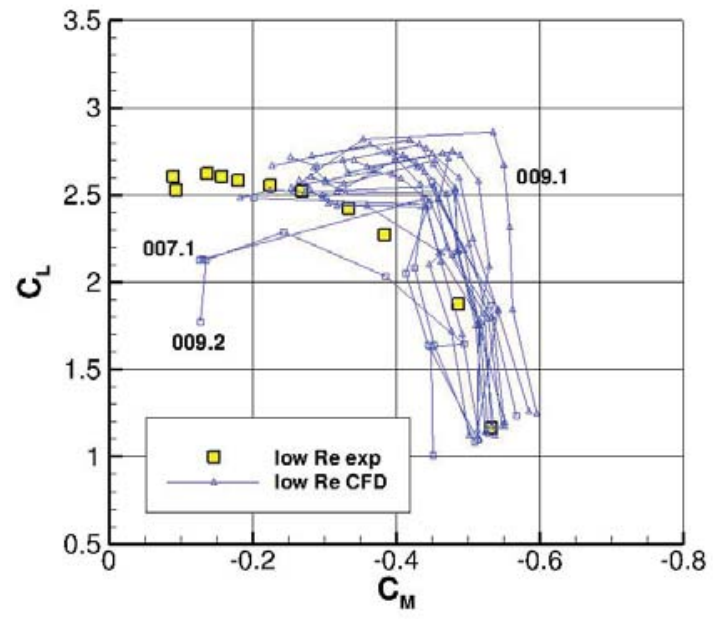

(e) $C_{M}, R e_{c}=1.35 \times 10^{6}$

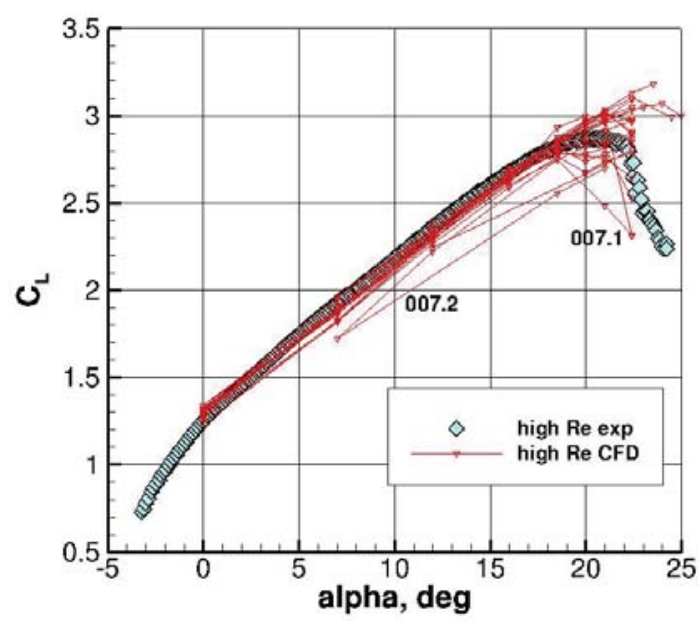

(b) $C_{L}, R e_{c}=15.1 \times 10^{6}$

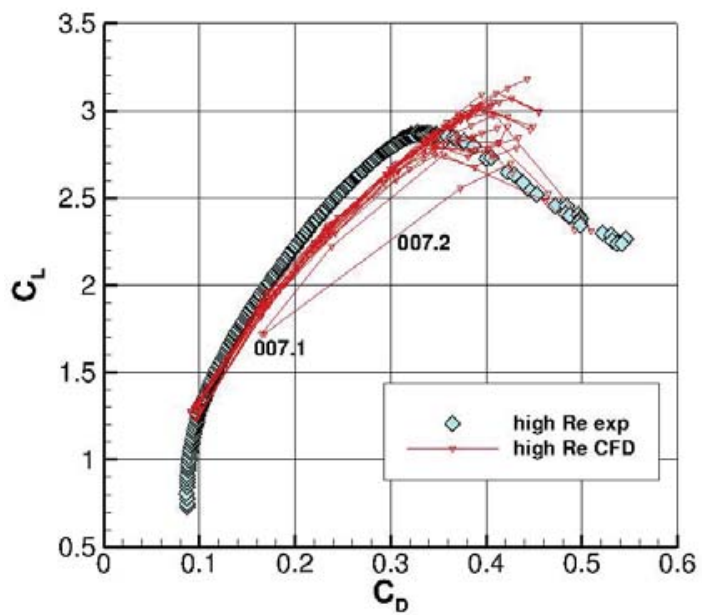

(d) $C_{D}, R e_{c}=15.1 \times 10^{6}$

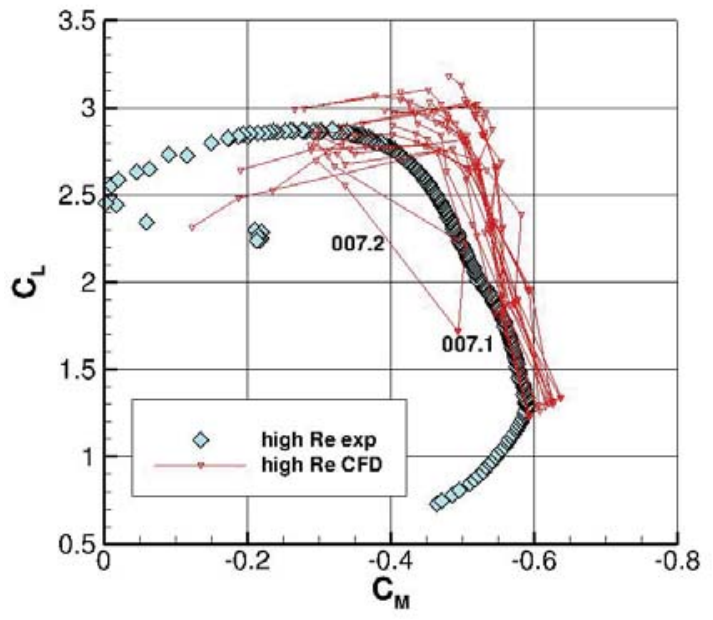

(f) $C_{M}, R e_{c}=15.1 \times 10^{6}$

Figure 7. Case 2 (medium grids with with brackets) forces and pitching moments. 


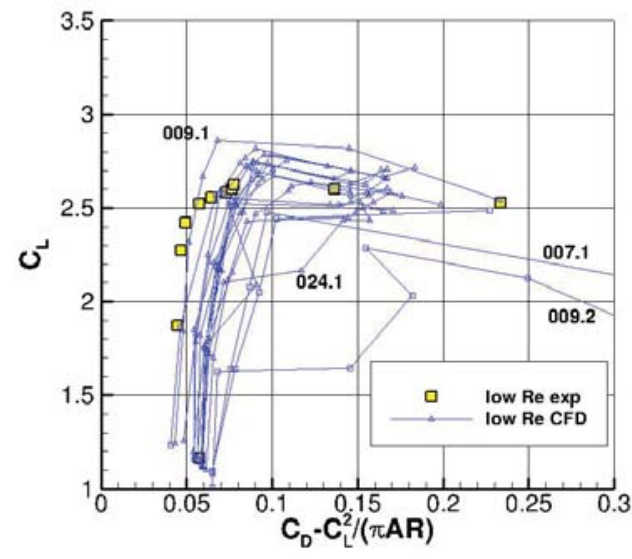

(a) $R e_{c}=1.35 \times 10^{6}$

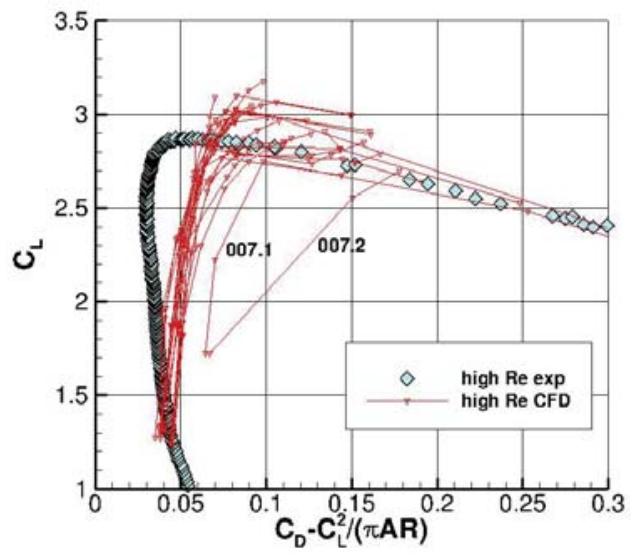

(b) $R e_{c}=15.1 \times 10^{6}$

Figure 8. $C_{D p}$ curves for Case 2 (medium grids with brackets).

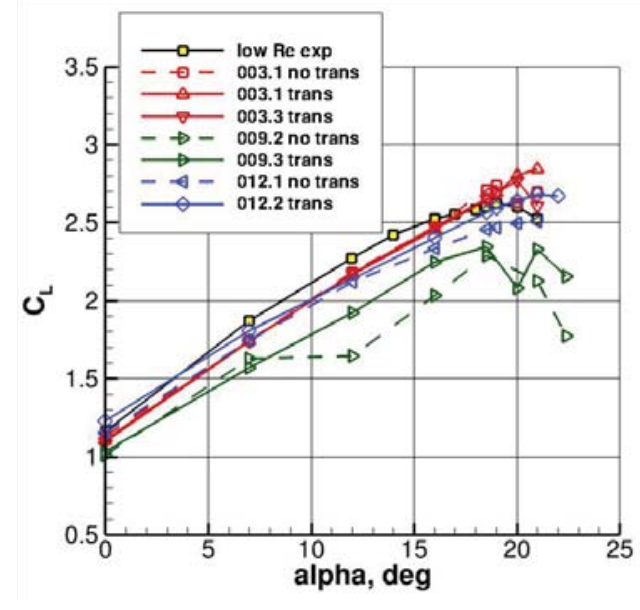

(a) $C_{L}$

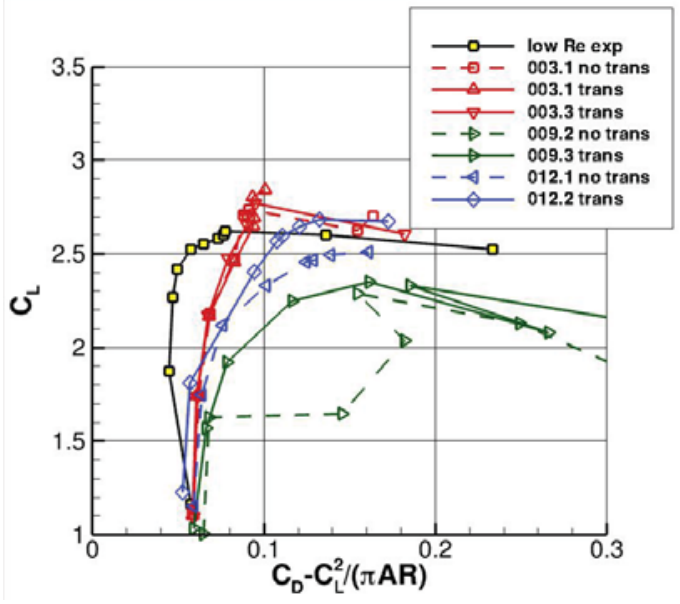

(b) $C_{D p}$

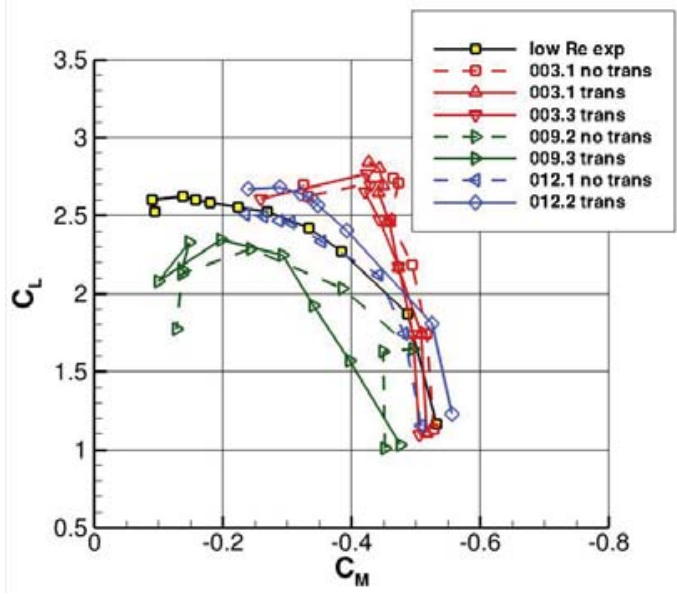

(c) $C_{M}$

Figure 9. Effects of including transition on forces and pitching moments, $R e_{c}=1.35 \times 10^{6}$ (medium grids with brackets). 


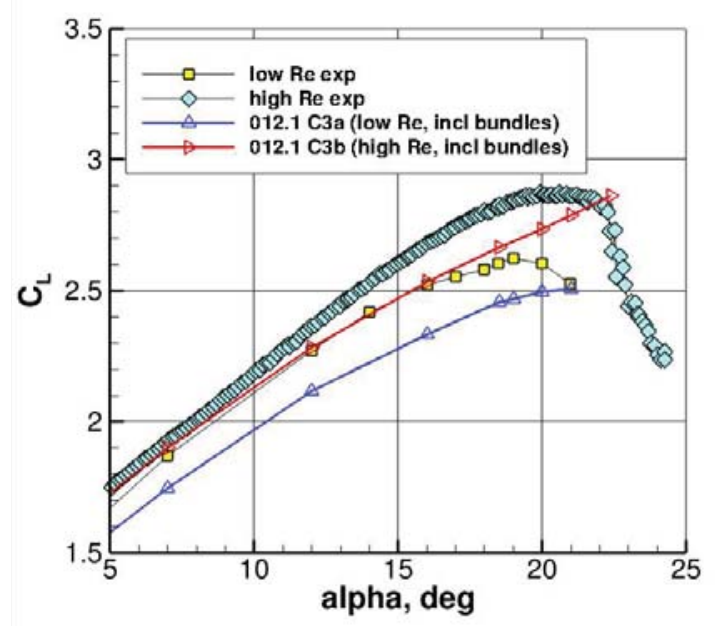

(a) Participant 012

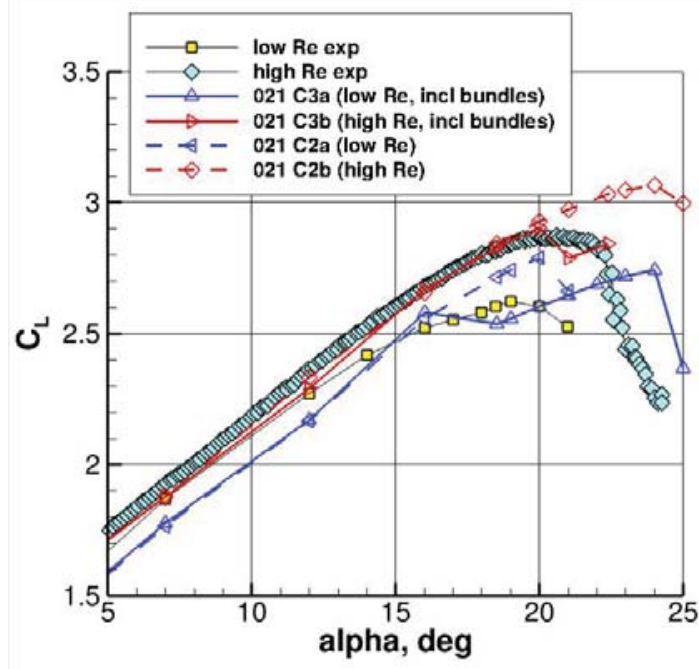

(b) Participant 021

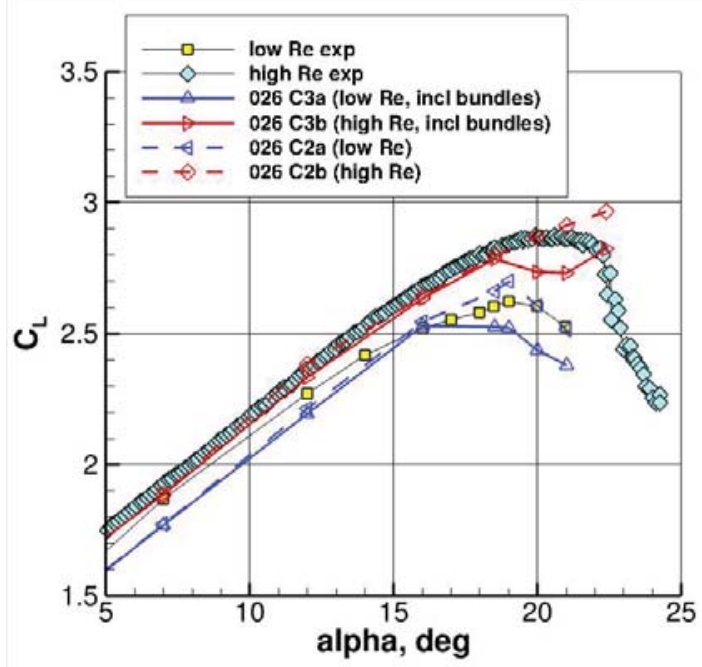

(c) Participant 026

Figure 10. Effect of including pressure tube bundles on $C_{L}$ (medium grids with brackets). 


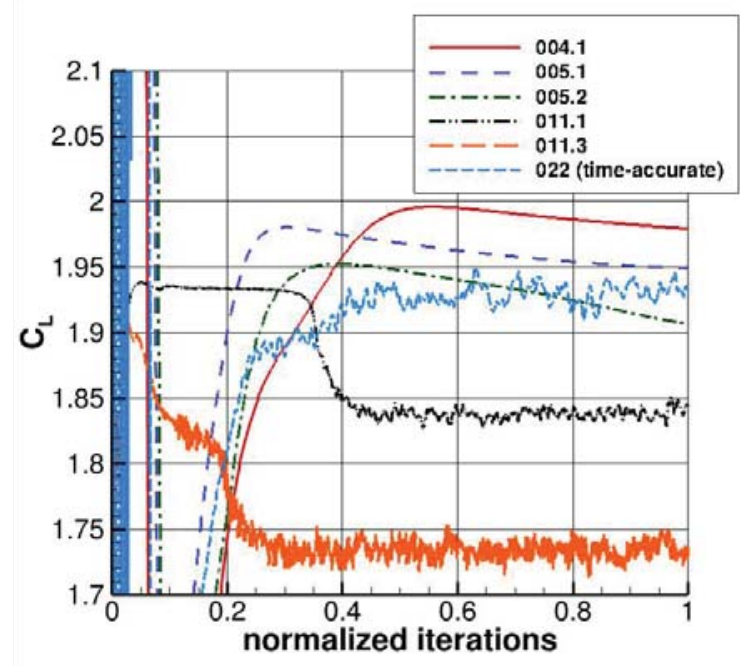

(a) Lift coefficient

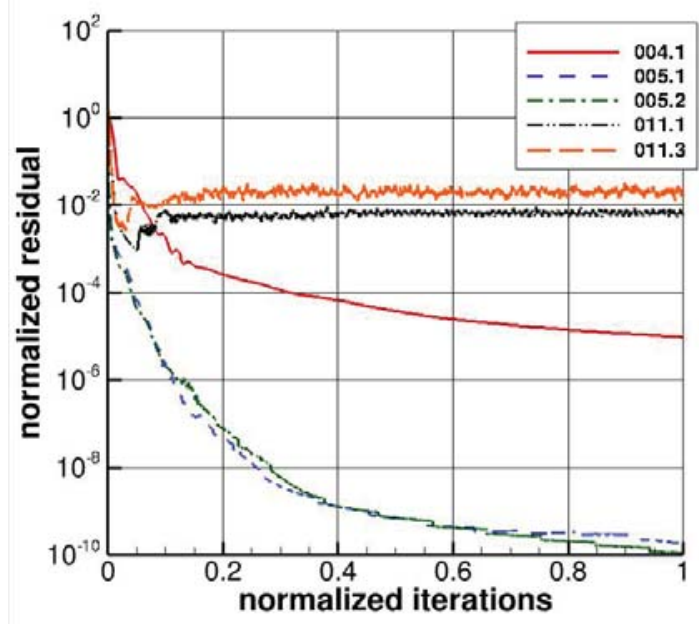

(b) Normalized density equation residual

Figure 11. Case 1 (no brackets, high $R e$ ) $\alpha=7^{\circ}$ iterative convergence information for entries with $C_{L}$ still changing at final iteration.

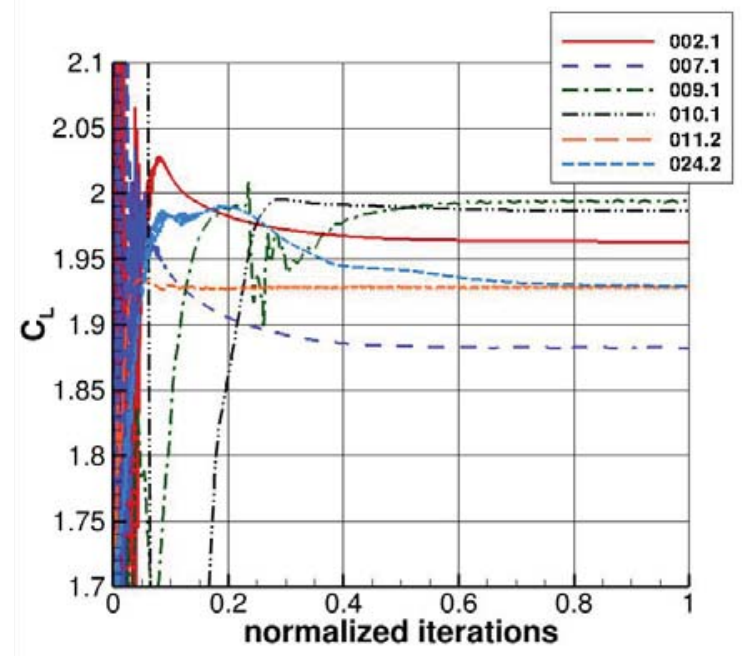

(a) Lift coefficient

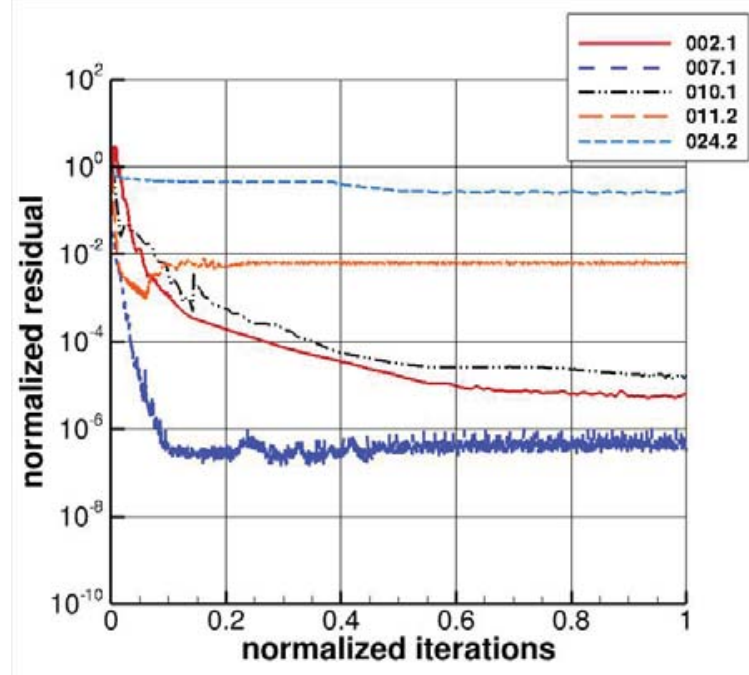

(b) Normalized density equation residual

Figure 12. Case 1 (no brackets, high $R e$ ) $\alpha=7^{\circ}$ representative iterative convergence information for entries with $C_{L}$ relatively flat at final iteration (all entries not shown). 


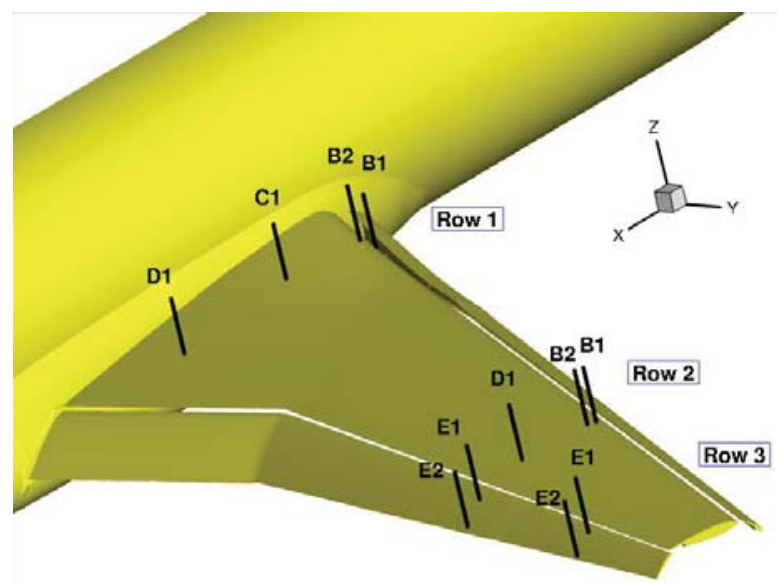

(a) Sketch showing locations of velocity profiles

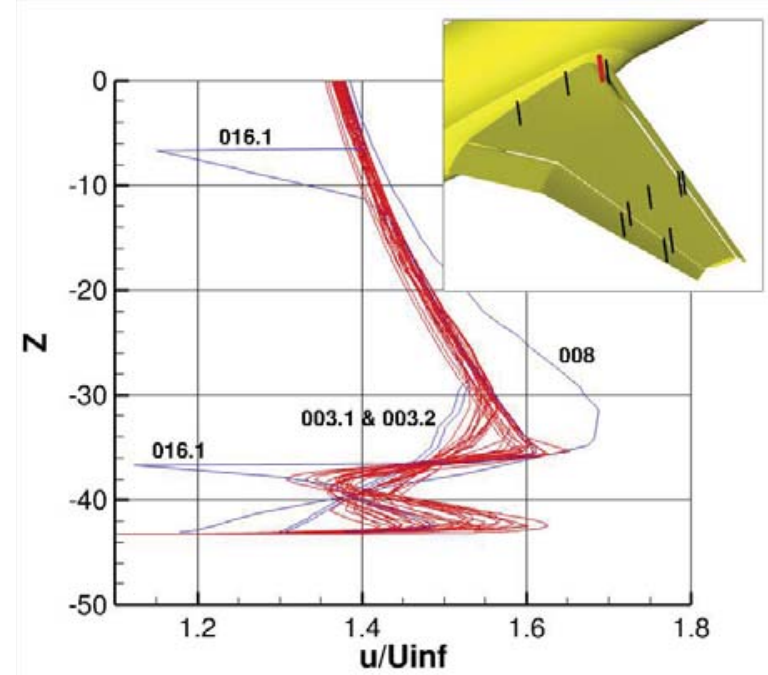

(c) Location 1B2

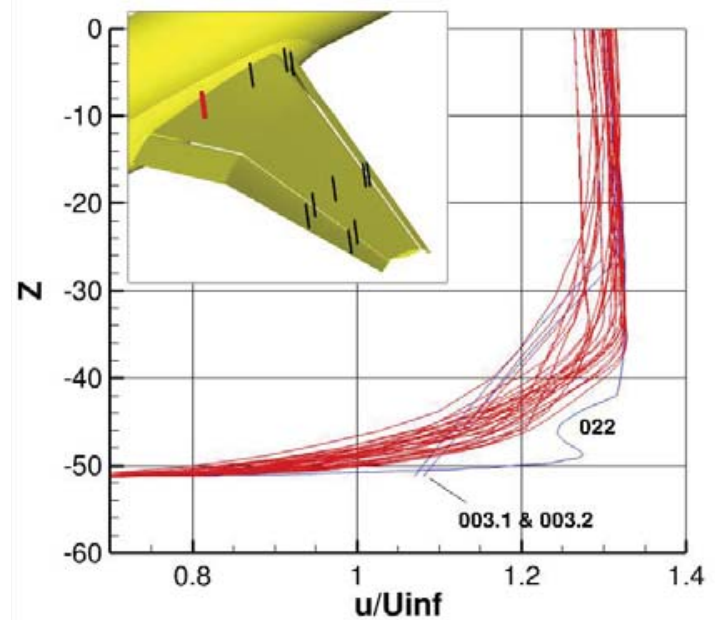

(e) Location 1D1

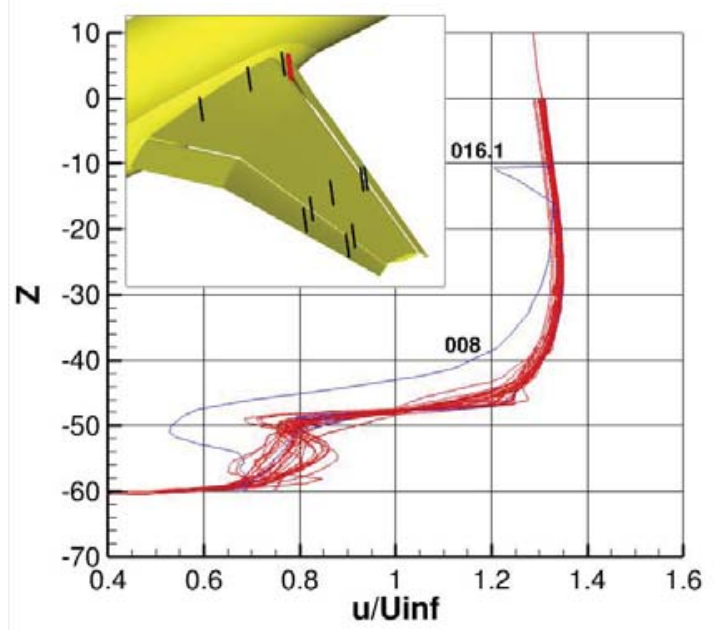

(b) Location 1B1

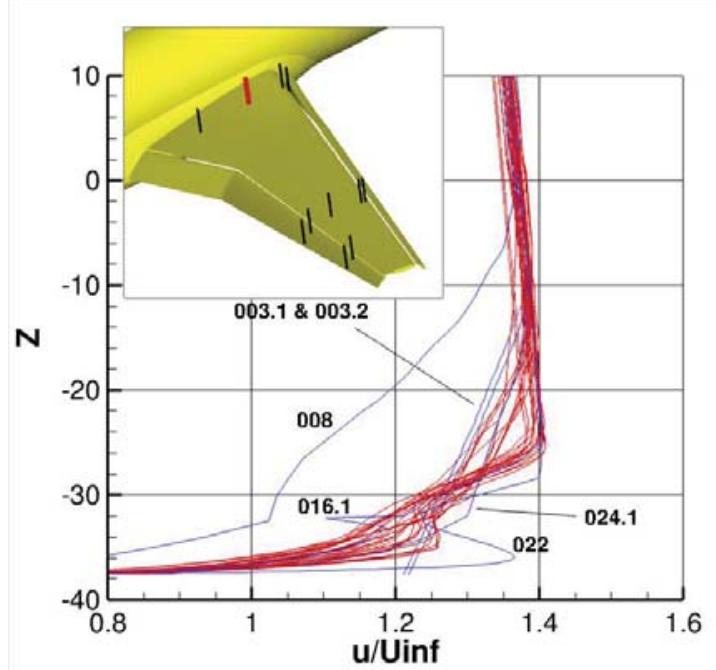

(d) Location 1C1

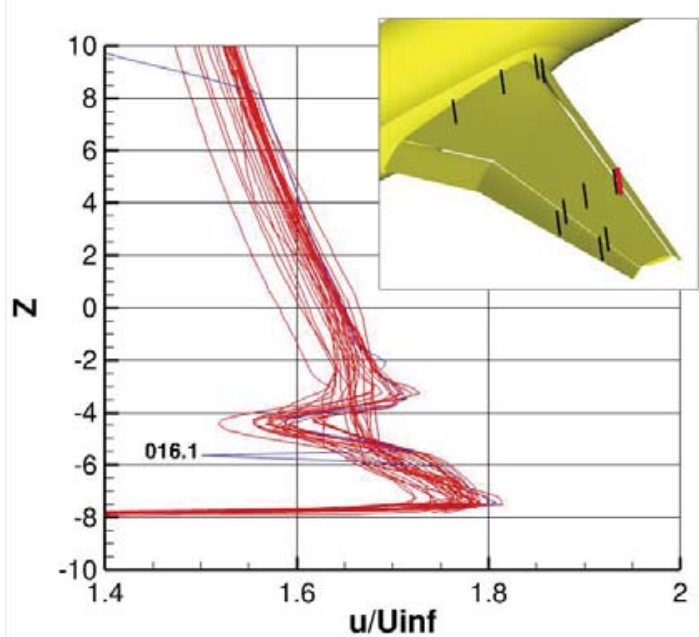

(f) Location 2B1

Figure 13. Case 1 (no brackets, high $R e$ ) $\alpha=7^{\circ}$ velocity profiles from CFD results on medium grids (part 1); obvious outliers denoted by blue lines. 


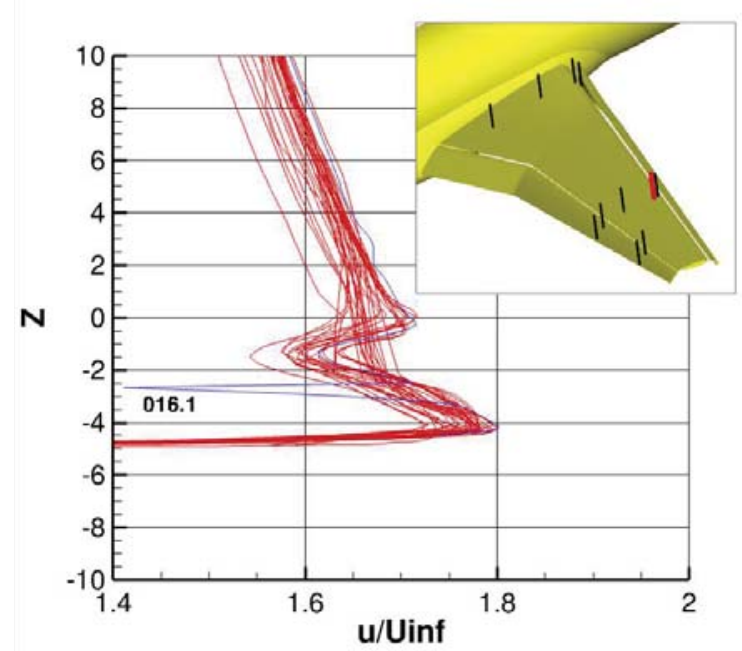

(a) Location 2B2

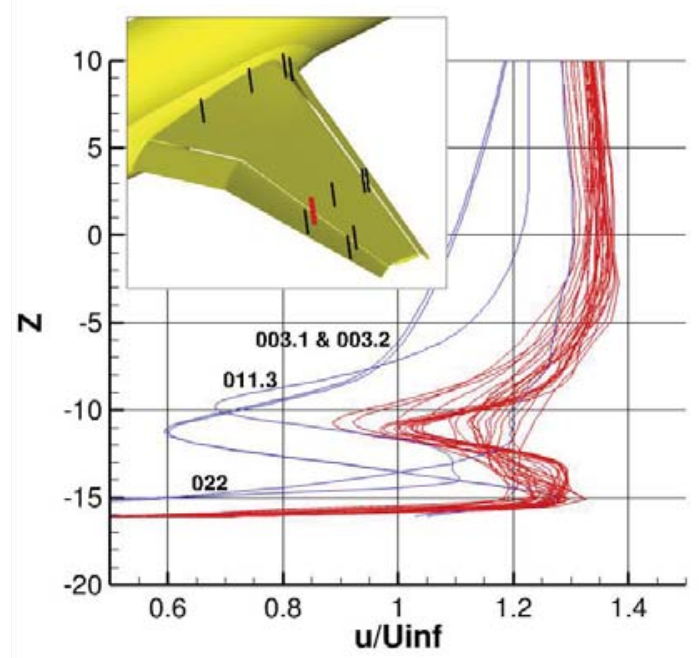

(c) Location 2E1

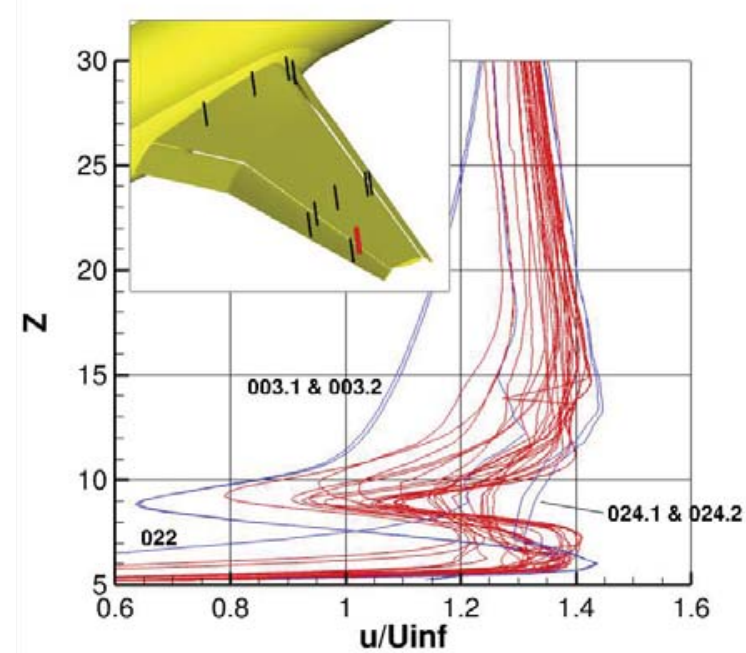

(e) Location 3E1

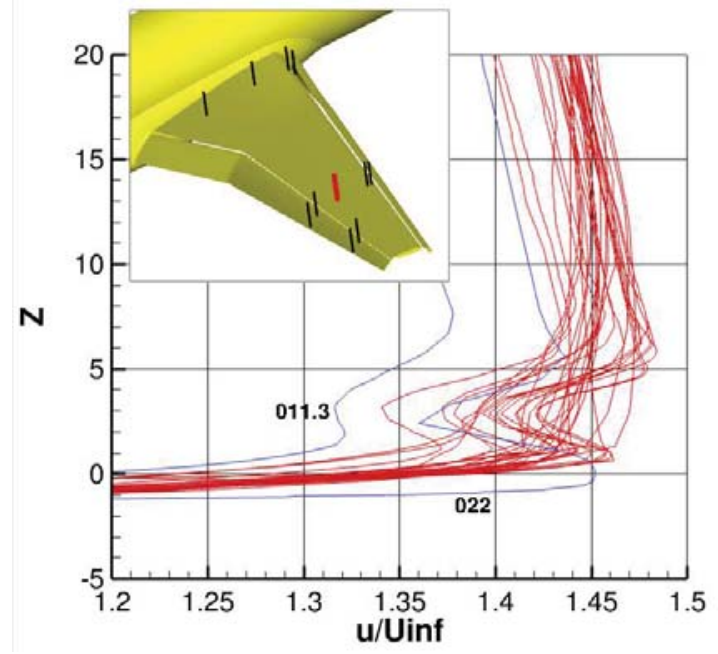

(b) Location 2D1

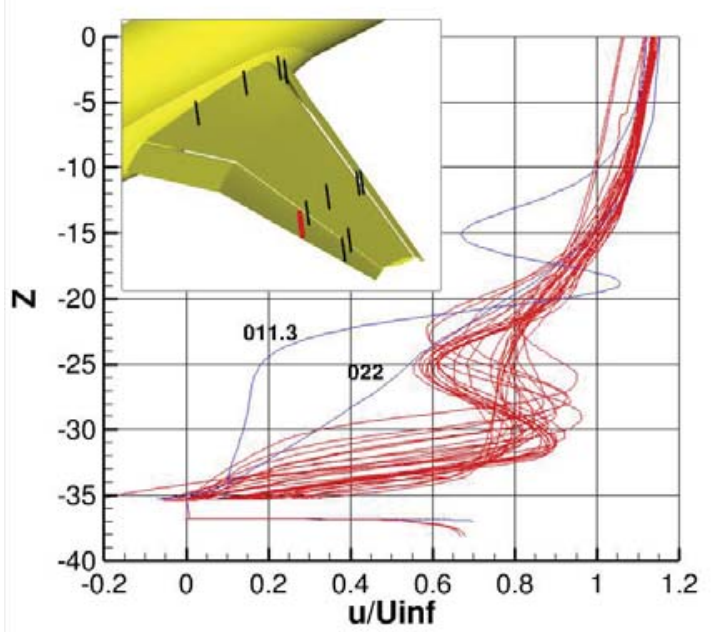

(d) Location 2E2

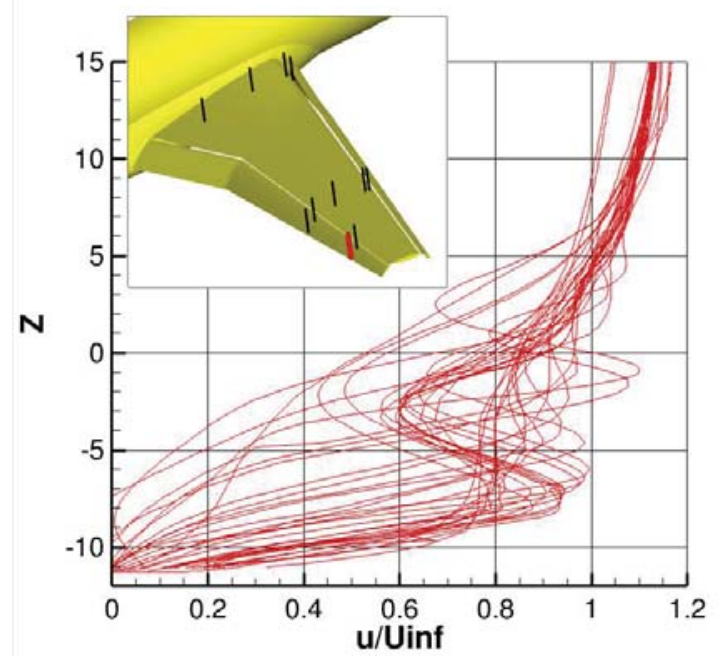

(f) Location 3E2

Figure 14. Case 1 (no brackets, high $R e$ ) $\alpha=7^{\circ}$ velocity profiles from CFD results on medium grids (part 2); obvious outliers denoted by blue lines. 


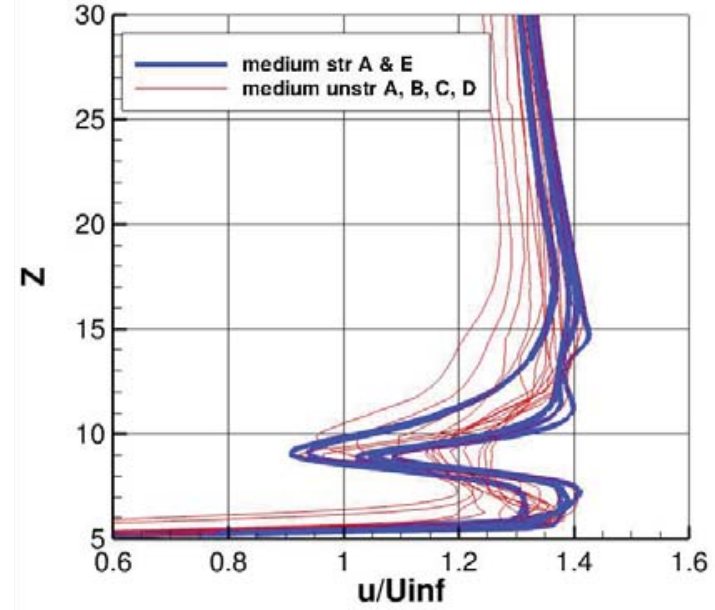

(a) All medium grid results, with structured results on medium A and E grids highlighted in bold blue

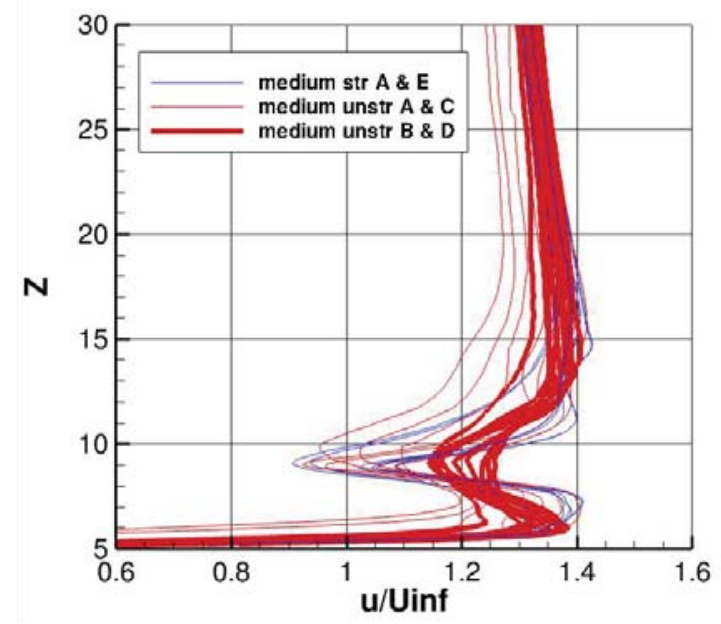

(c) All medium grid results, with unstructured results on medium B and $\mathrm{D}$ grids highlighted in bold red

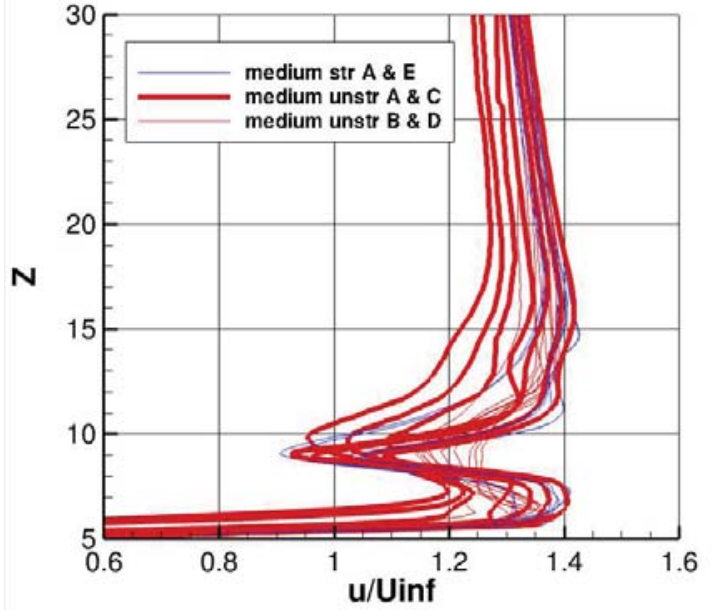

(b) All medium grid results, with unstructured results on medium A and $\mathrm{C}$ grids highlighted in bold red

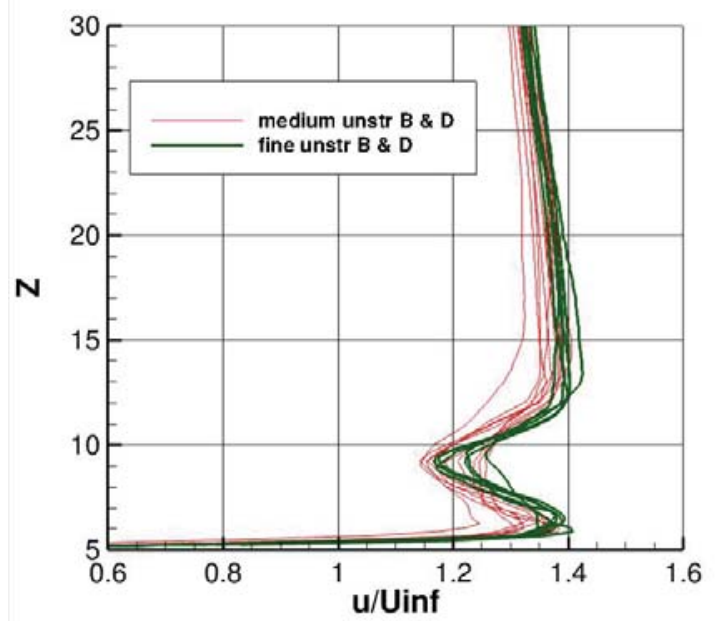

(d) Medium and fine unstructured B and D grid results only

Figure 15. Case 1 (no brackets, high $R e$ ) $\alpha=7^{\circ}$ velocity profiles at location 3E1, highlighted by grid type (outliers removed). 


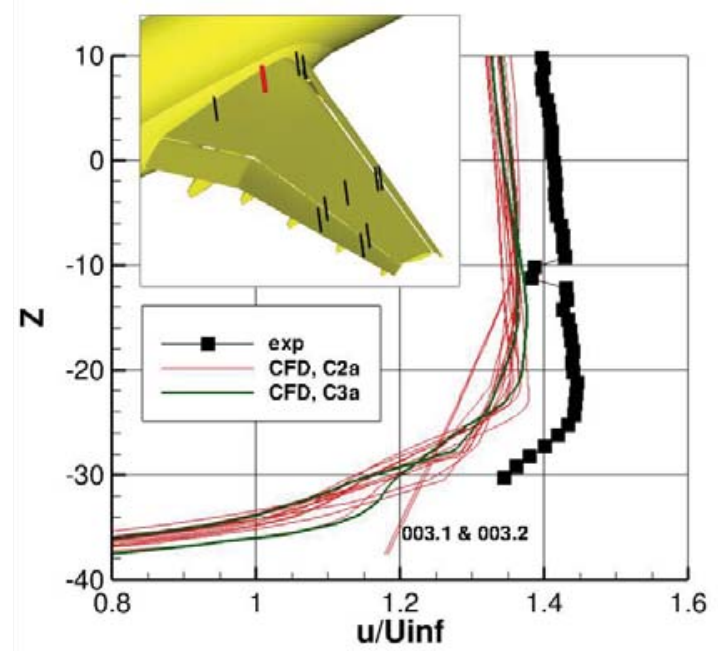

(a) Location $1 \mathrm{C} 1$ at $\alpha=7^{\circ}$

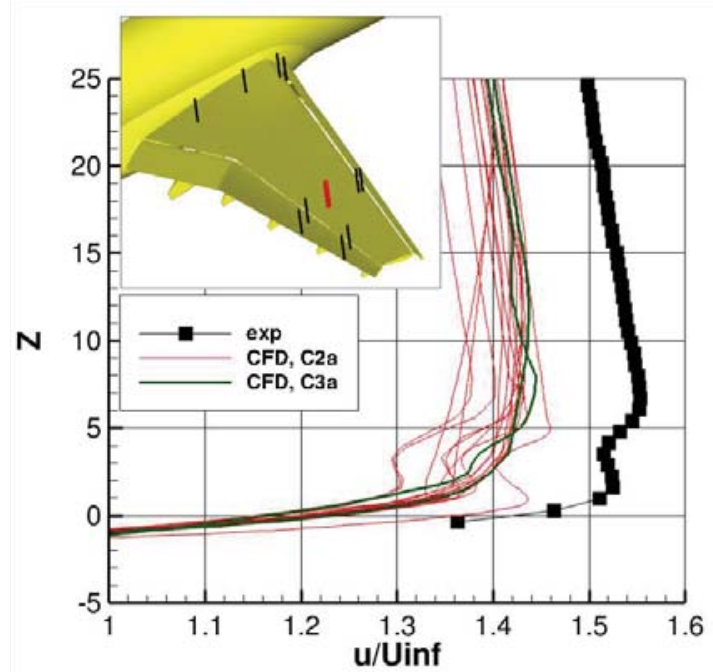

(c) Location 2D1 at $\alpha=7^{\circ}$

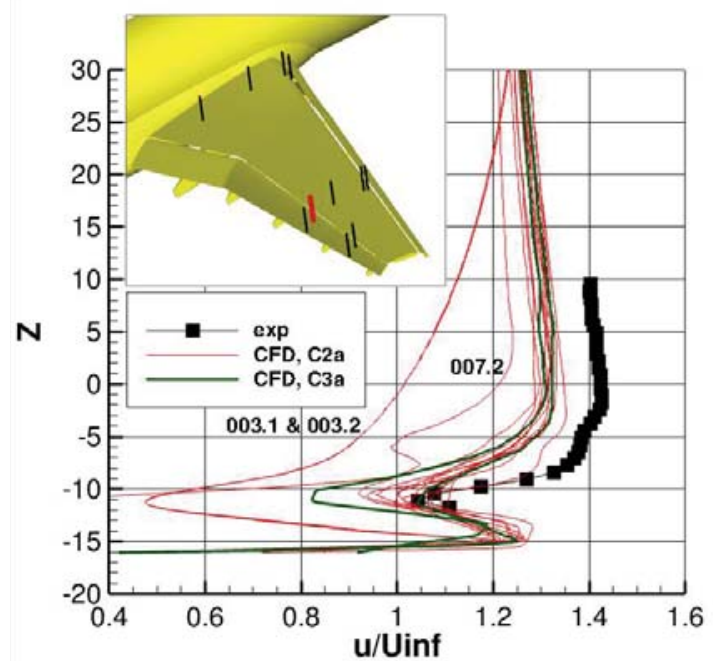

(e) Location 2E1 at $\alpha=7^{\circ}$

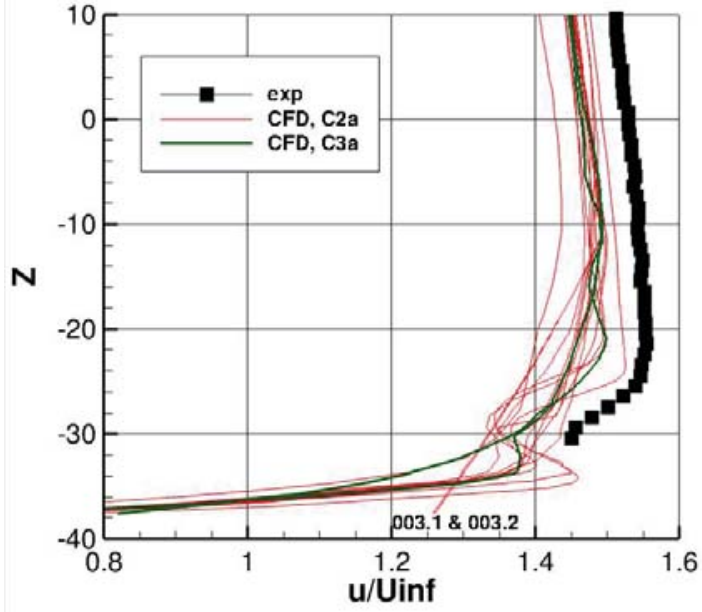

(b) Location $1 \mathrm{C} 1$ at $\alpha=18.5^{\circ}$

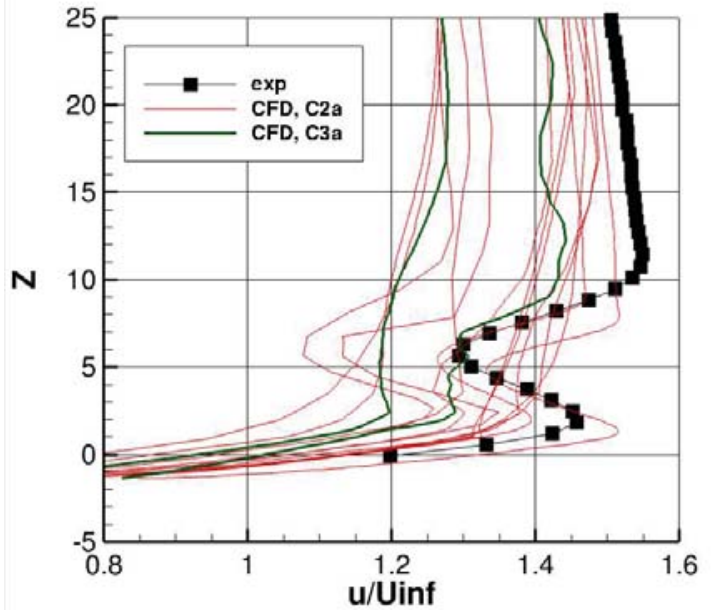

(d) Location 2D1 at $\alpha=18.5^{\circ}$

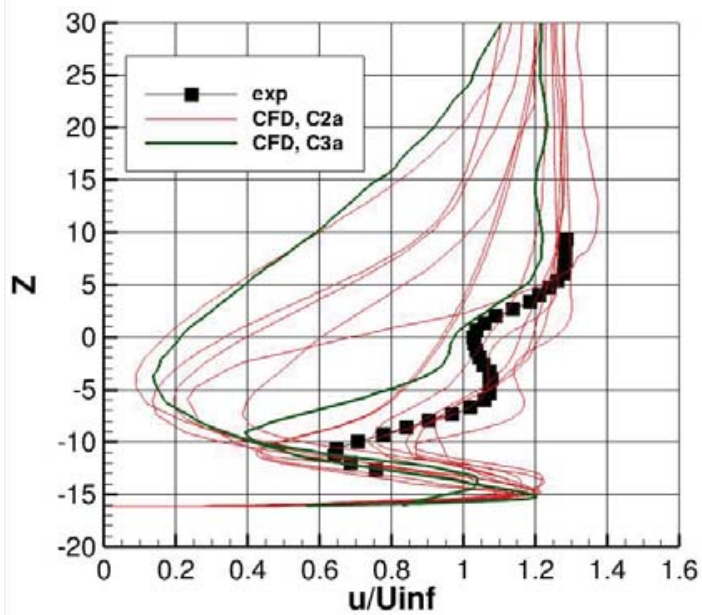

(f) Location 2E1 at $\alpha=18.5^{\circ}$

Figure 16. Case 2a (with brackets, low $R e$ ) and 3a (with brackets and bundles, low $R e$ ) velocity profiles at selected locations using medium grids. 


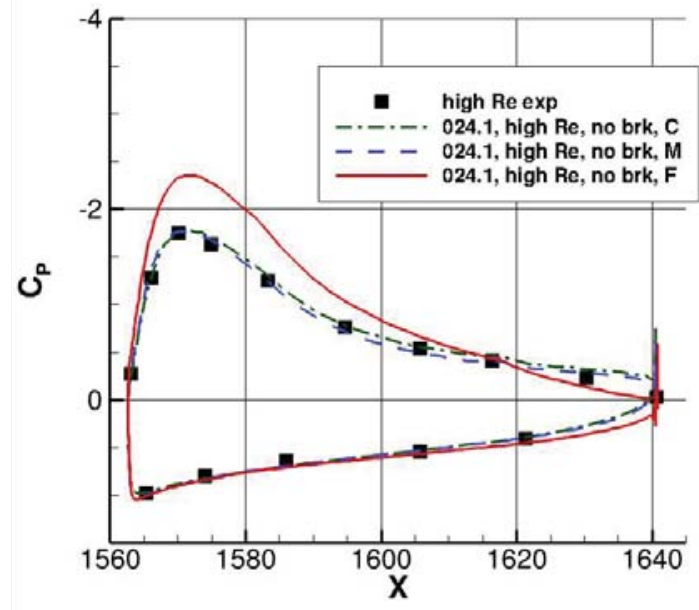

(a) Flap at $2 y / B=0.15$, no brackets

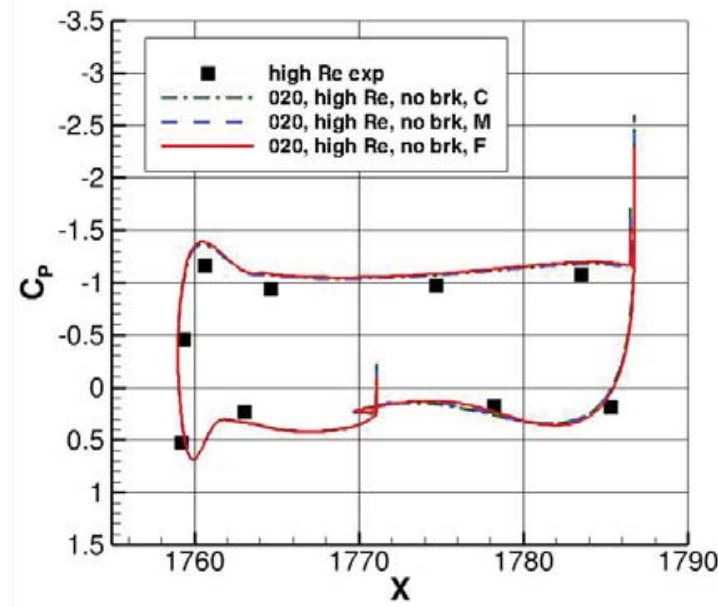

(c) Slat at $2 y / B=0.89$, no brackets

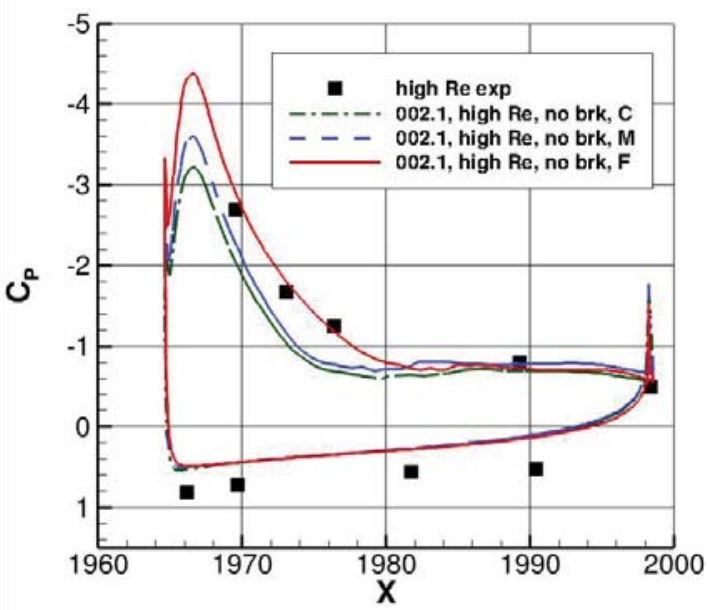

(e) Flap at $2 y / B=0.96$, no brackets

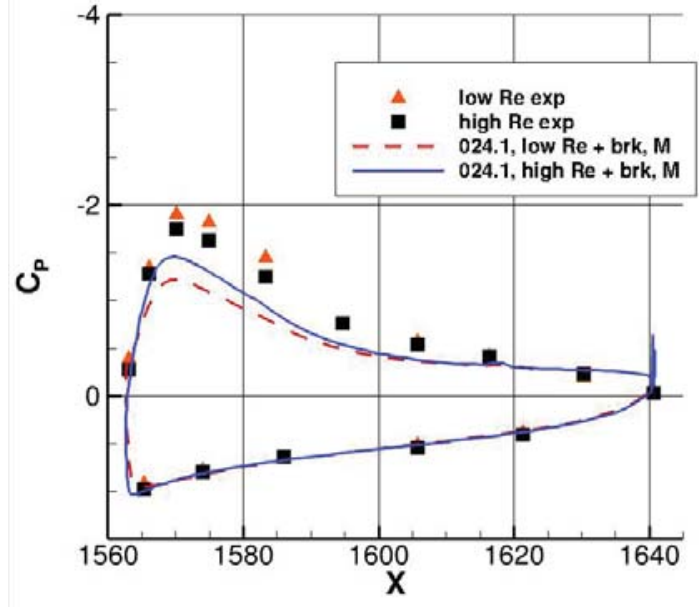

(b) Flap at $2 y / B=0.15$, including brackets

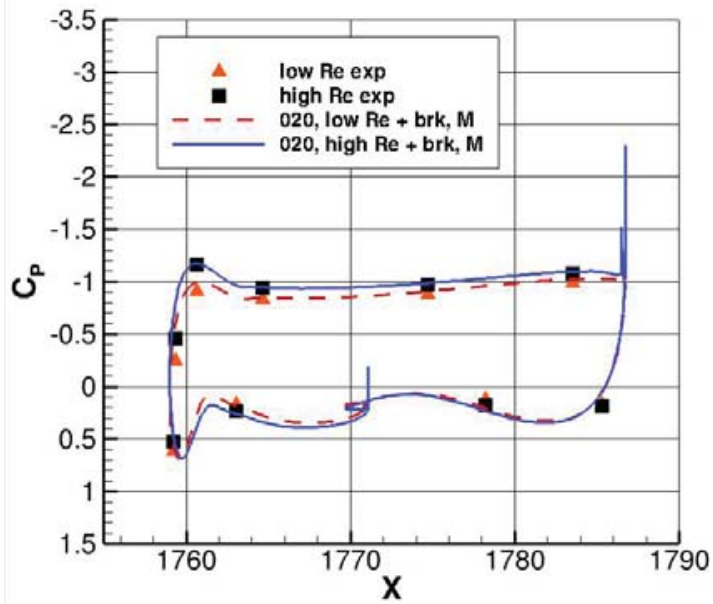

(d) Slat at $2 y / B=0.89$, including brackets

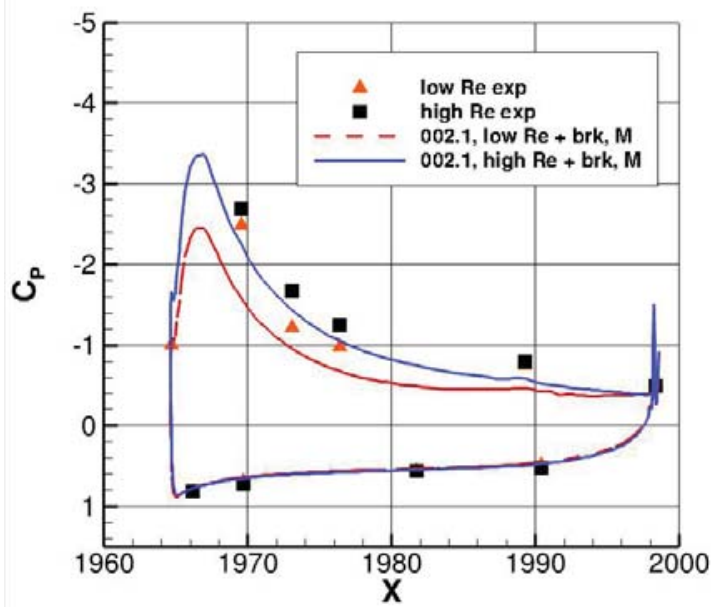

(f) Flap at $2 y / B=0.96$, including brackets

Figure 17. Sampling of typical surface pressure coefficients at $\alpha=7^{\circ}$. 


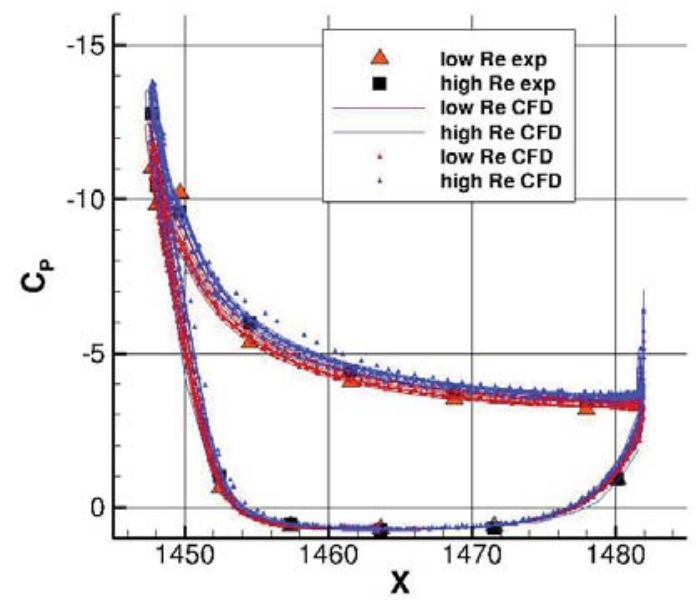

(a) Slat at $2 y / B=0.54$

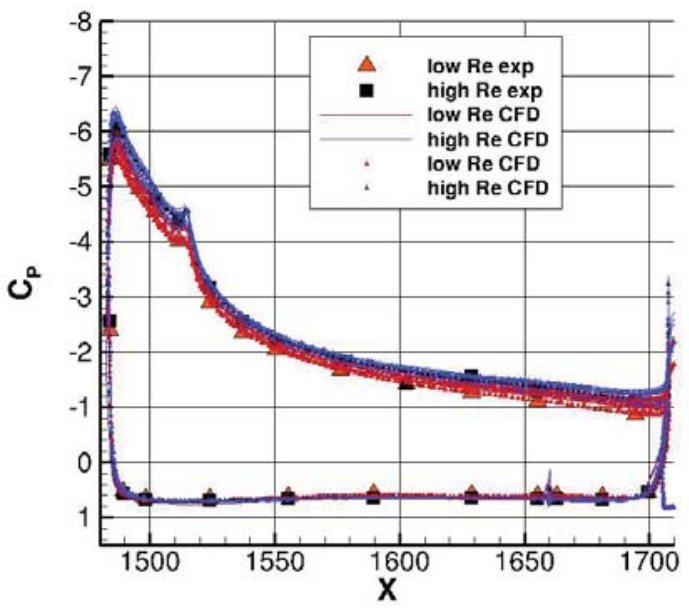

(c) Main at $2 y / B=0.54$

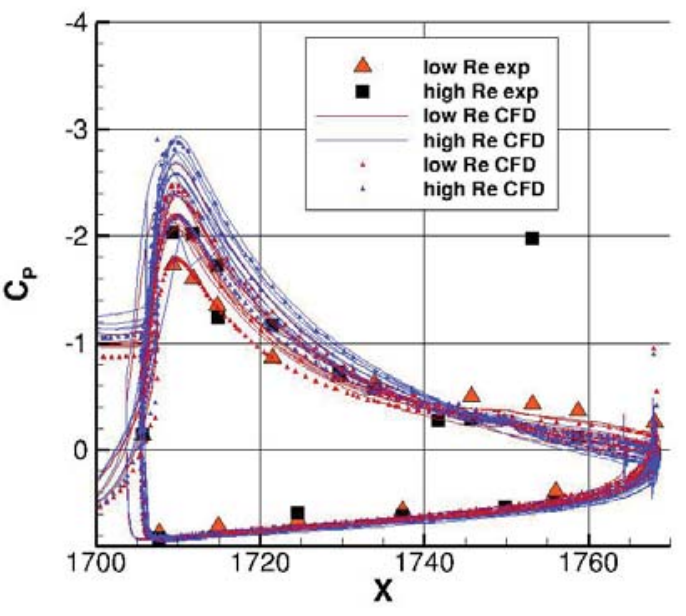

(e) Flap at $2 y / B=0.54$

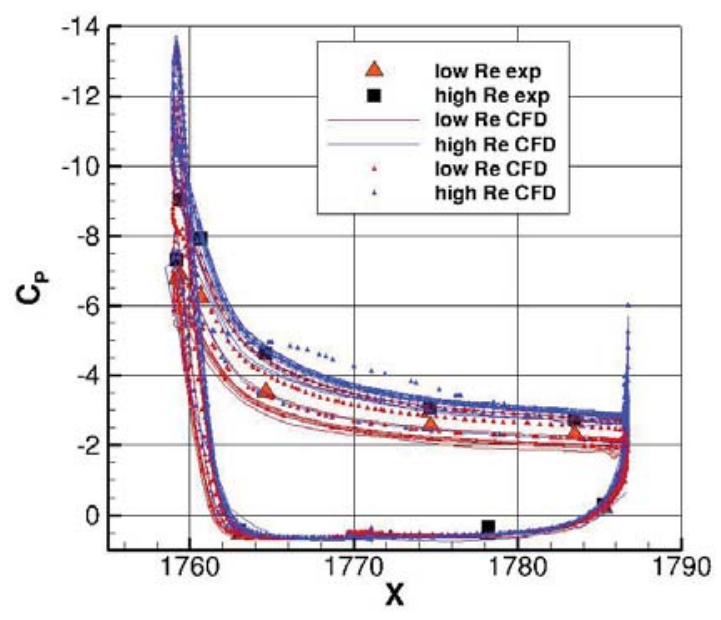

(b) Slat at $2 y / B=0.89$

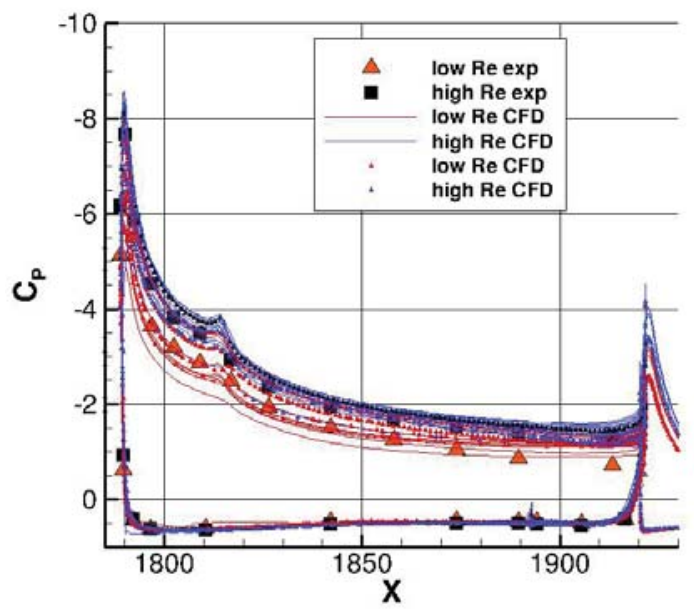

(d) Main at $2 y / B=0.89$

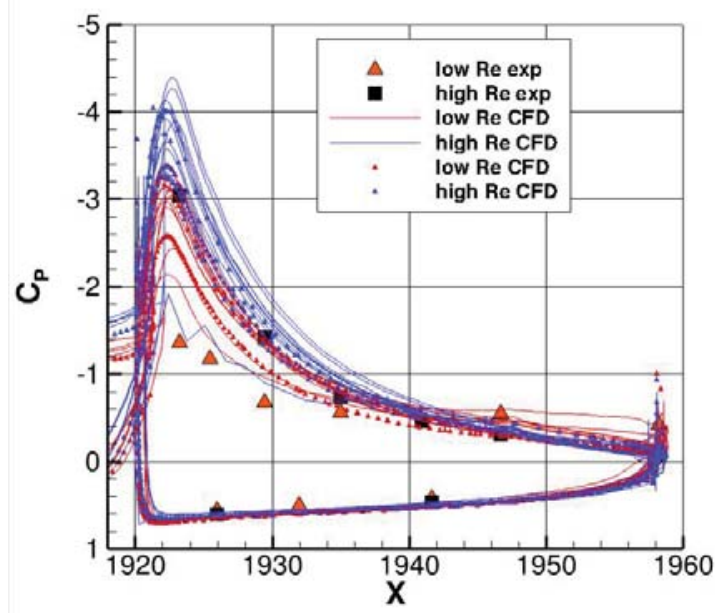

(f) Flap at $2 y / B=0.89$

Figure 18. Pressure coefficients at two span stations for $\alpha=20^{\circ}$ (with brackets included). 


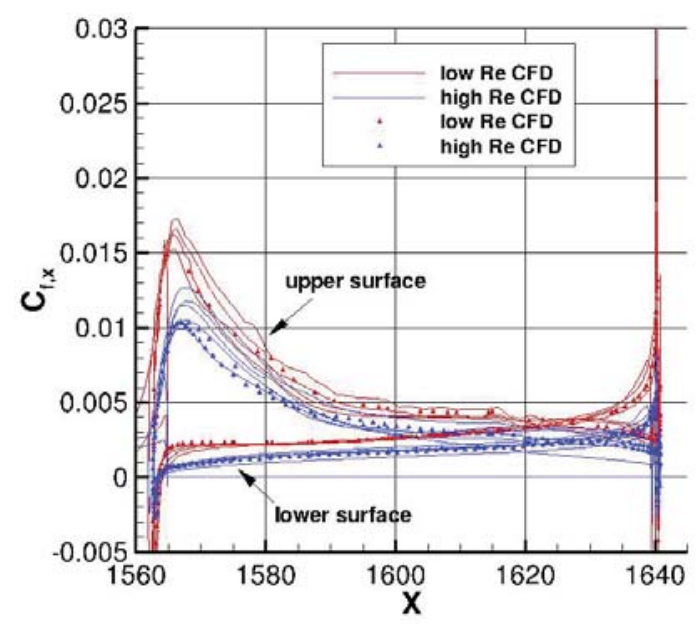

(a) Flap at $2 y / B=0.15, \alpha=7^{\circ}$

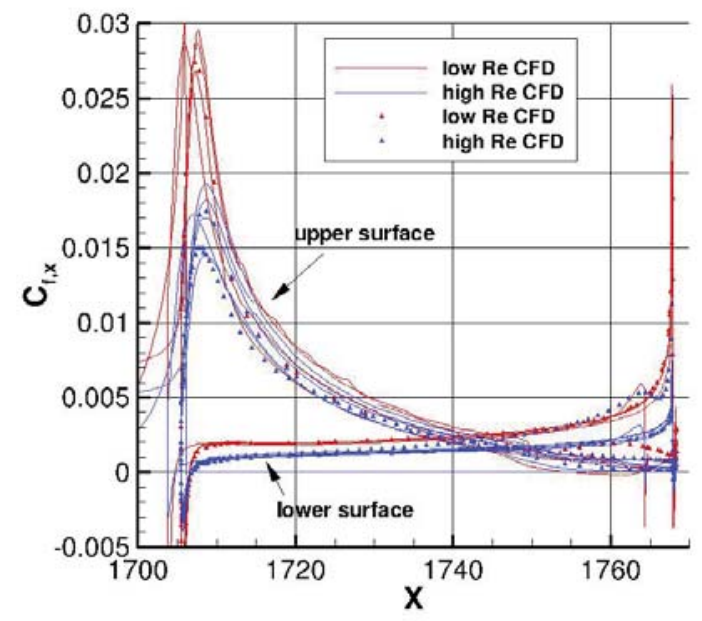

(c) Flap at $2 y / B=0.54, \alpha=7^{\circ}$

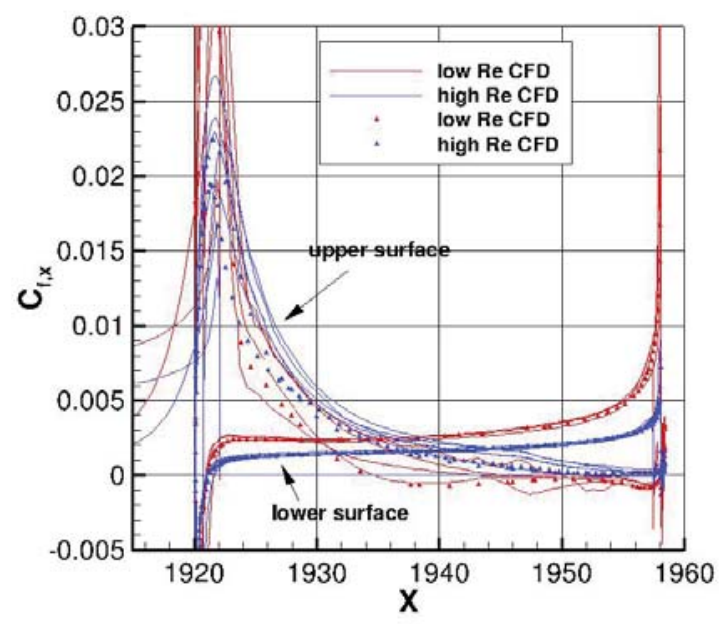

(e) Flap at $2 y / B=0.89, \alpha=7^{\circ}$

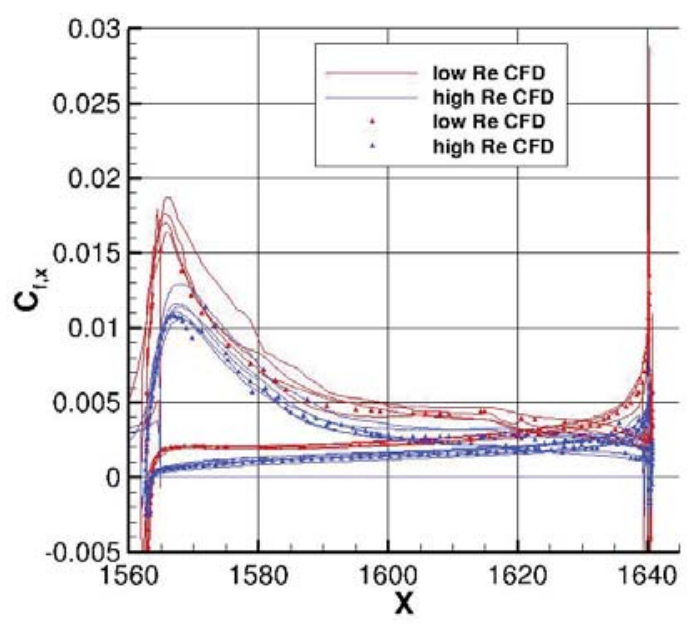

(b) Flap at $2 y / B=0.15, \alpha=16^{\circ}$

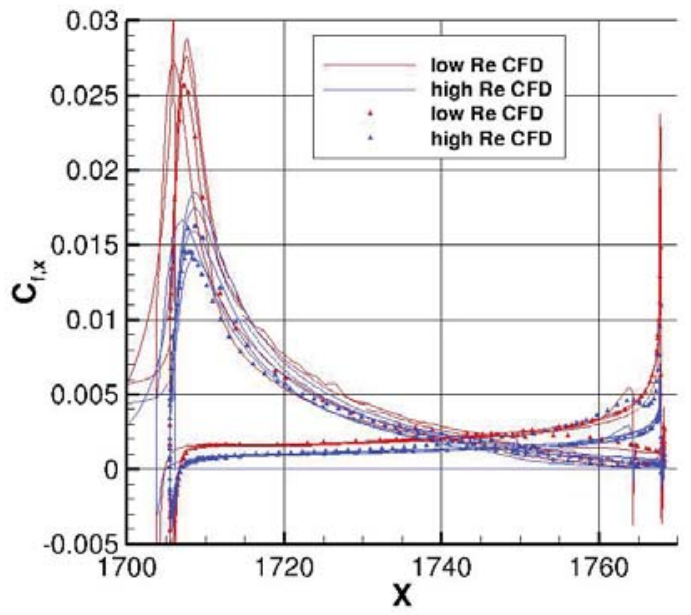

(d) Flap at $2 y / B=0.54, \alpha=16^{\circ}$

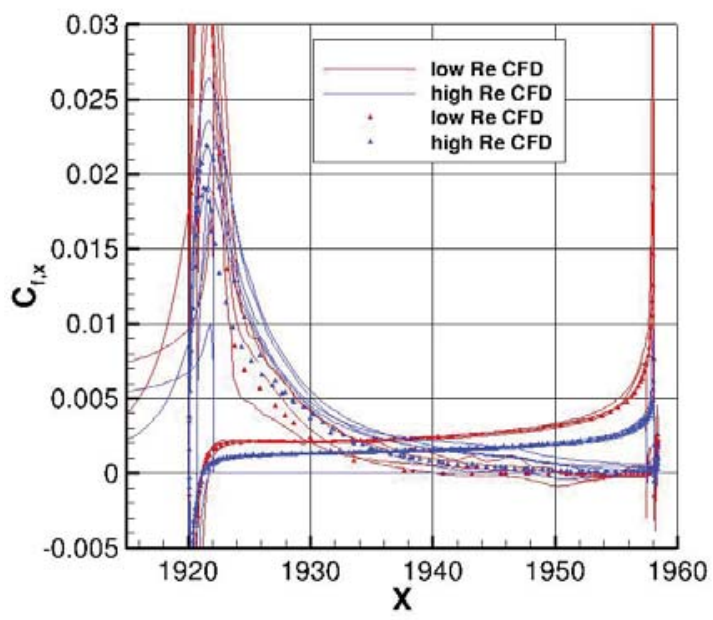

(f) Flap at $2 y / B=0.89, \alpha=16^{\circ}$

Figure 19. The $x$-component of skin friction coefficients on the flap at $\alpha=7^{\circ}$ and $16^{\circ}$ (with brackets included). 


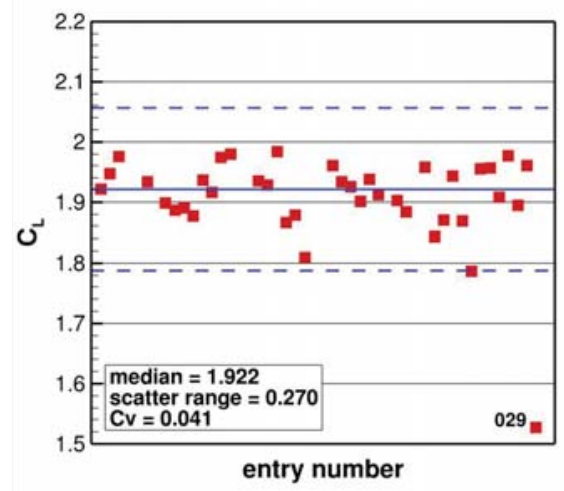

(a) $C_{L}$, coarse grid

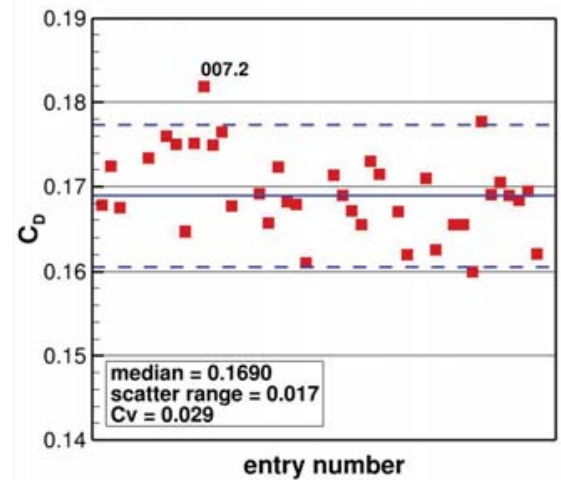

(d) $C_{D}$, coarse grid

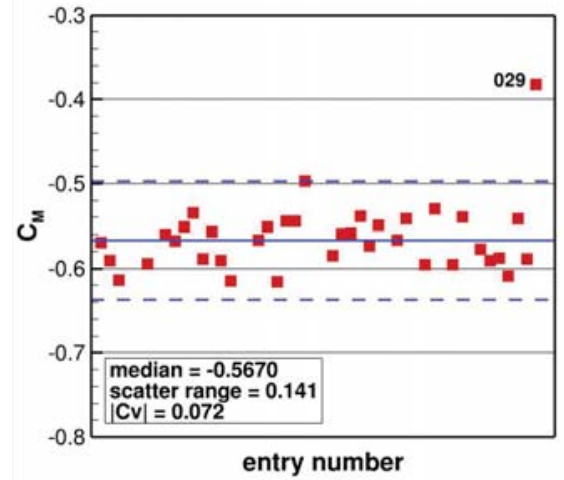

(g) $C_{M}$, coarse grid

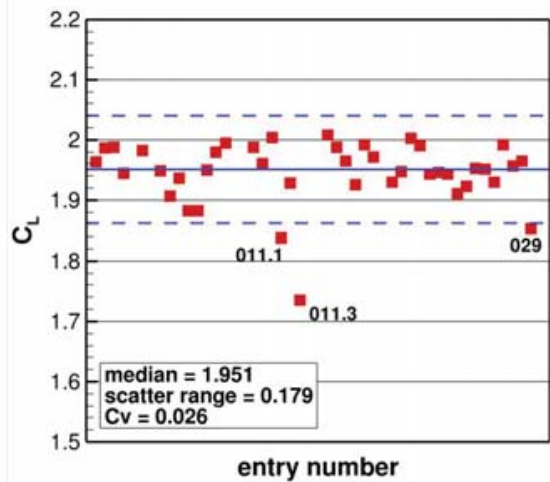

(b) $C_{L}$, medium grid

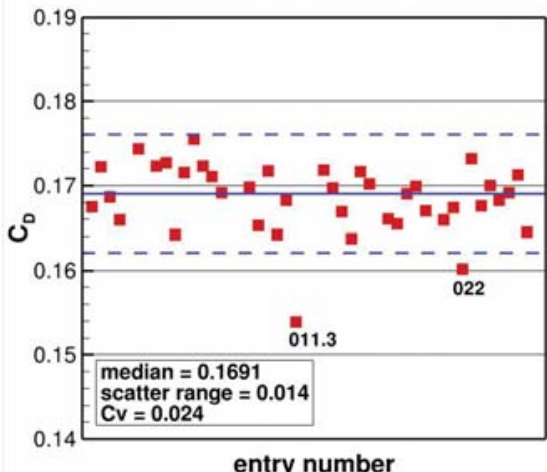

(e) $C_{D}$, medium grid

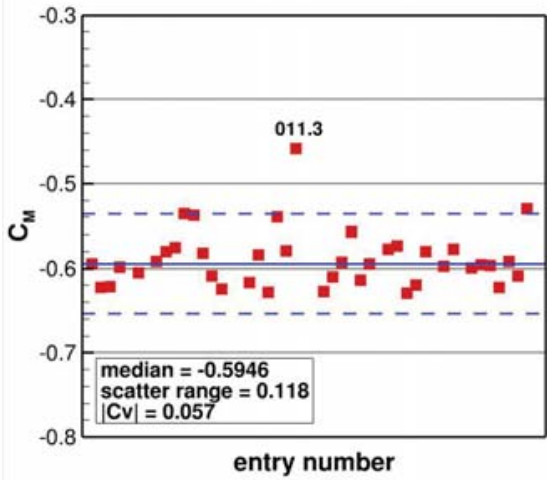

(h) $C_{M}$, medium grid

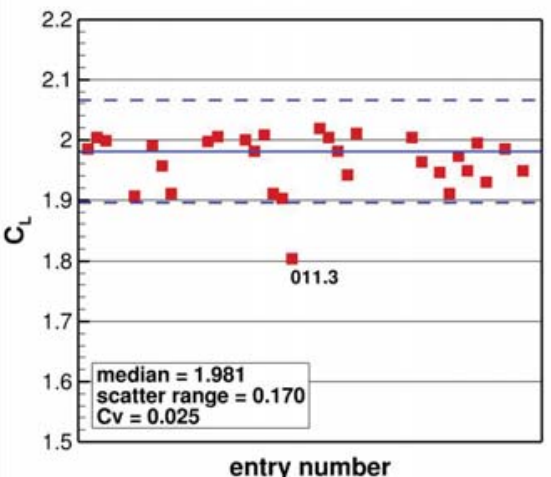

(c) $C_{L}$, fine grid

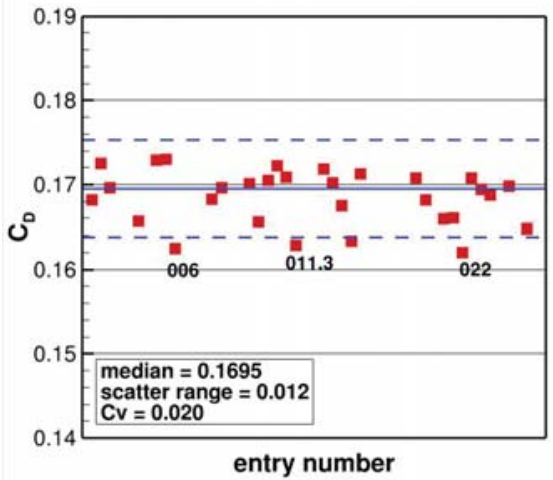

(f) $C_{D}$, fine grid

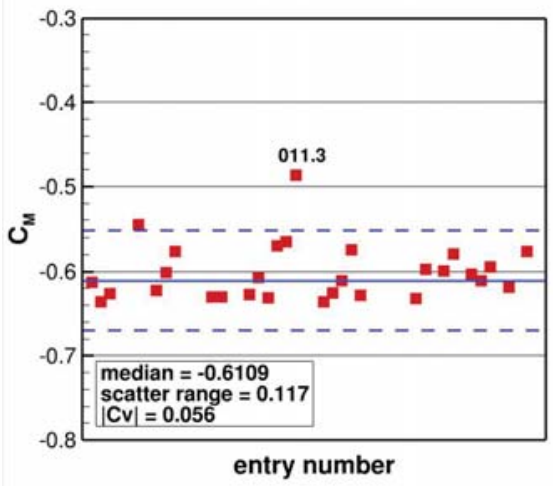

(i) $C_{M}$, fine grid

Figure 20. Statistical analysis of Case 1 (no brackets, high $R e$ ), $\alpha=7^{\circ}$; solid blue line is median of data, dashed blue lines are scatter limits. 


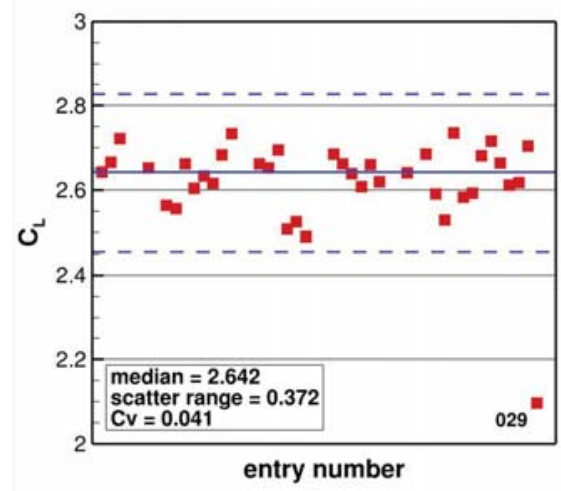

(a) $C_{L}$, coarse grid

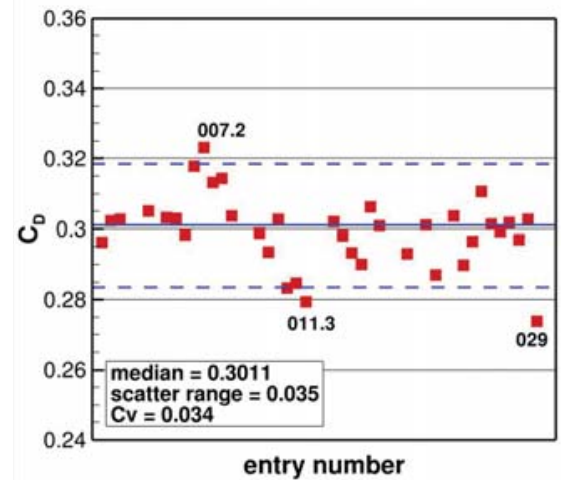

(d) $C_{D}$, coarse grid

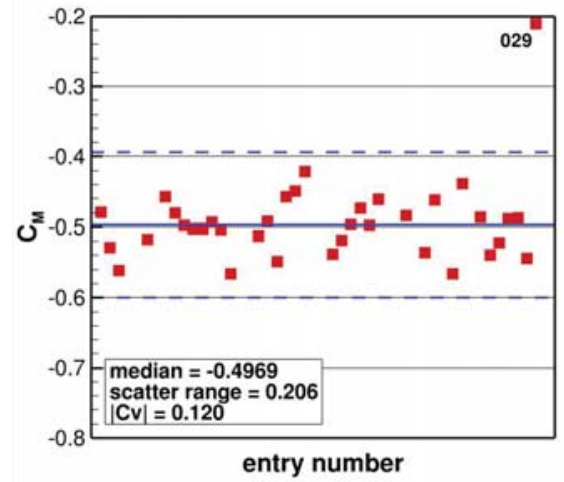

(g) $C_{M}$, coarse grid

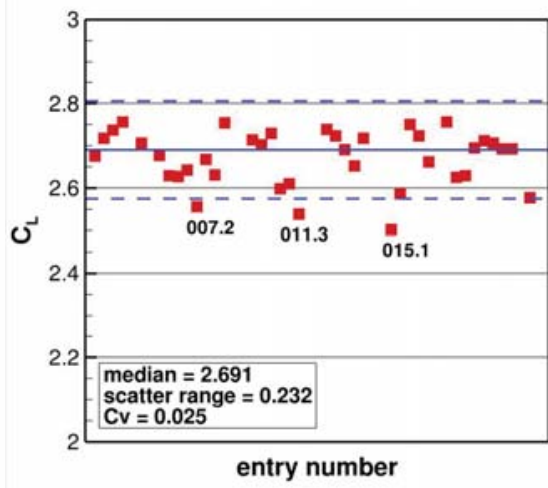

(b) $C_{L}$, medium grid

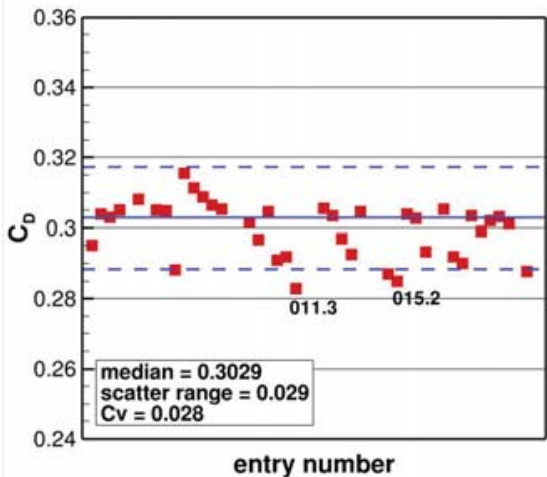

(e) $C_{D}$, medium grid

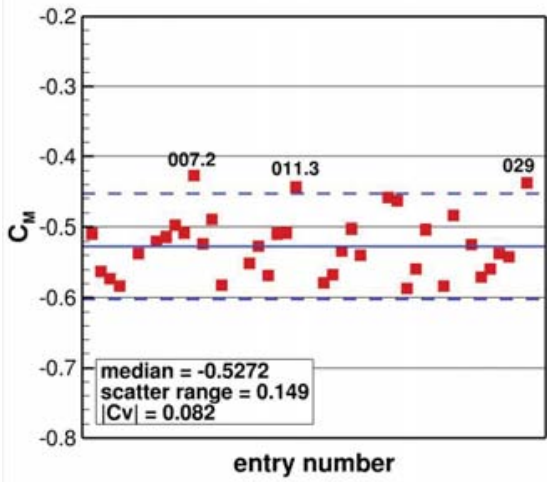

(h) $C_{M}$, medium grid

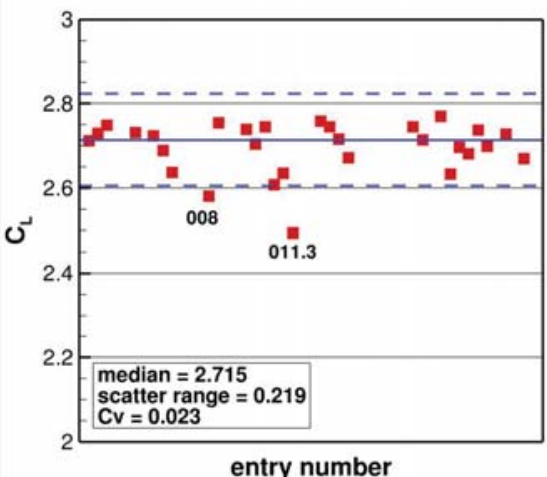

(c) $C_{L}$, fine grid

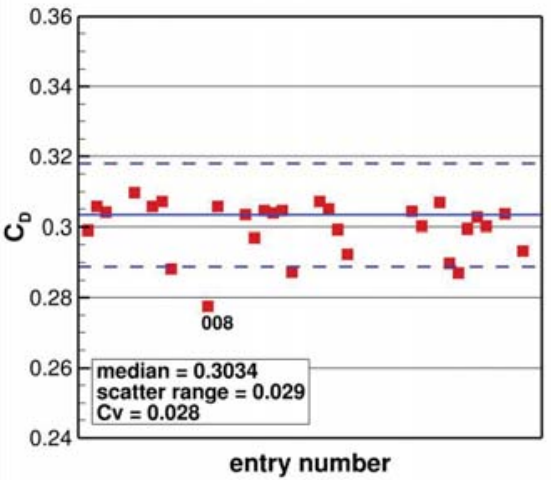

(f) $C_{D}$, fine grid

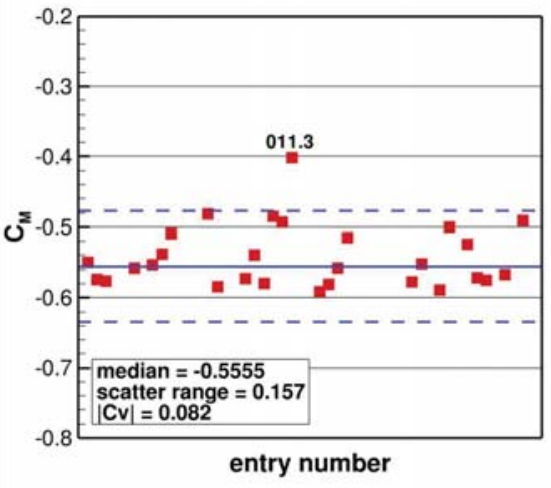

(i) $C_{M}$, fine grid

Figure 21. Statistical analysis of Case 1 (no brackets, high $R e$ ), $\alpha=16^{\circ}$; solid blue line is median of data, dashed blue lines are scatter limits. 


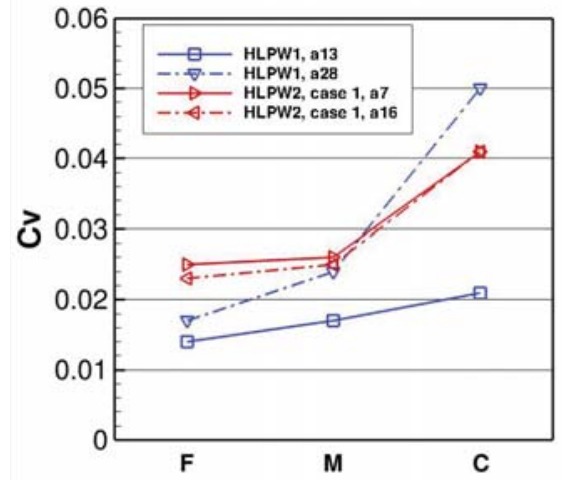

(a) Lift coefficient

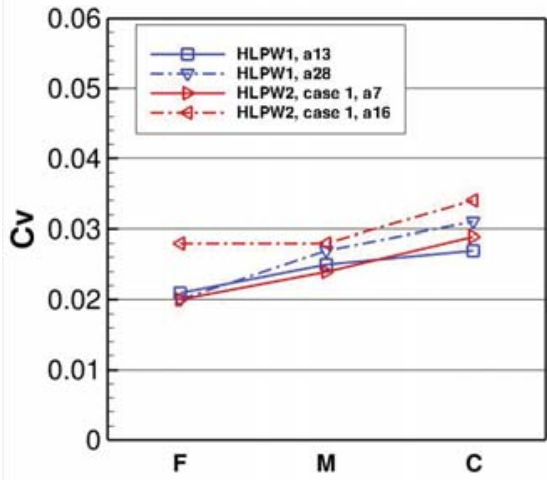

(b) Drag coefficient

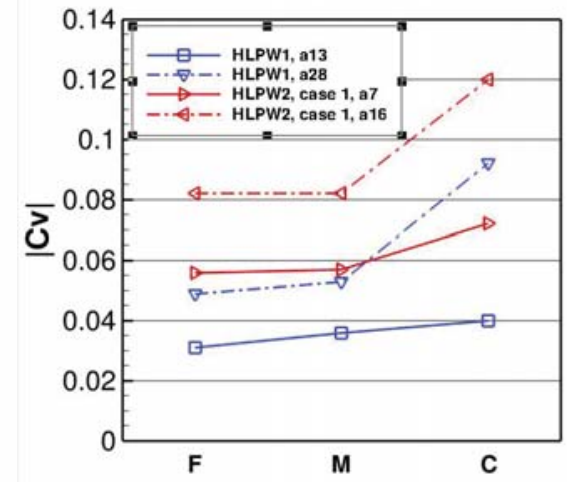

(c) Moment coefficient

Figure 22. Summary of coefficients of variation, in comparison with HiLiftPW-1 results.

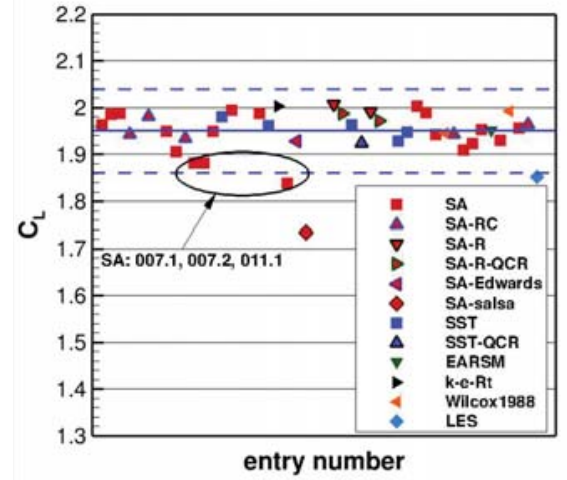

(a) $\alpha=7^{\circ}$, separated by turbulence model

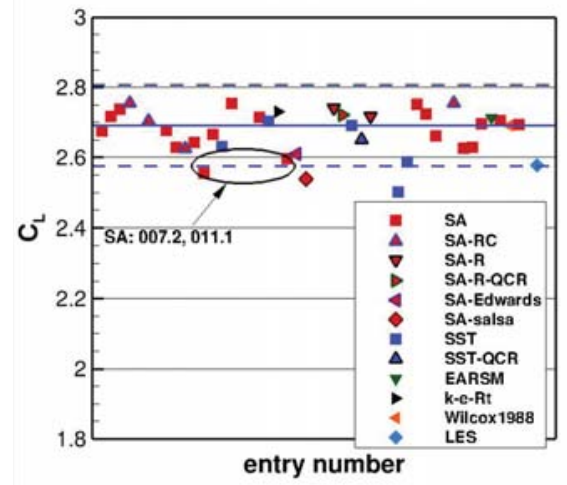

(d) $\alpha=16^{\circ}$, separated by turbulence model

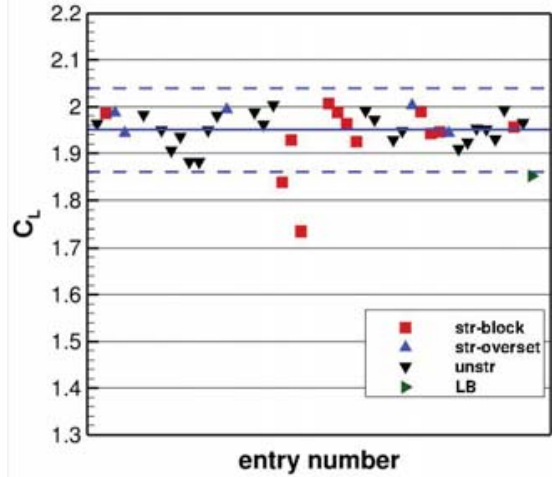

(b) $\alpha=7^{\circ}$, separated by grid type

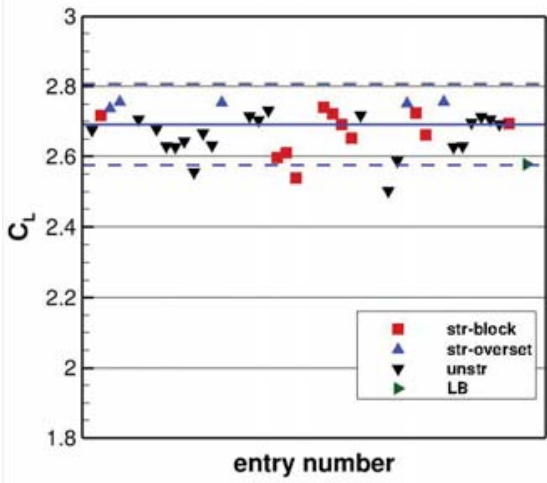

(e) $\alpha=16^{\circ}$, separated by grid type

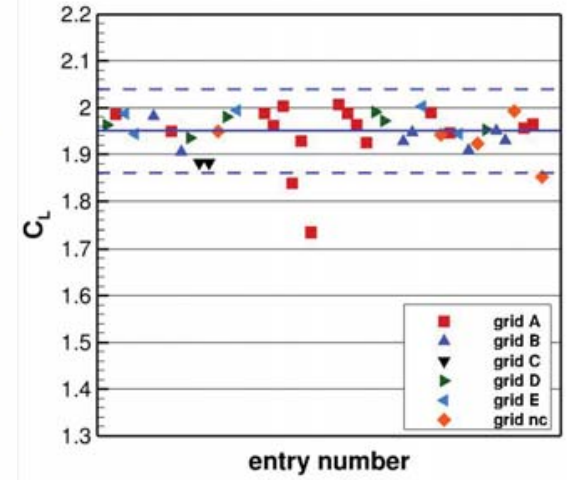

(c) $\alpha=7^{\circ}$, separated by grid

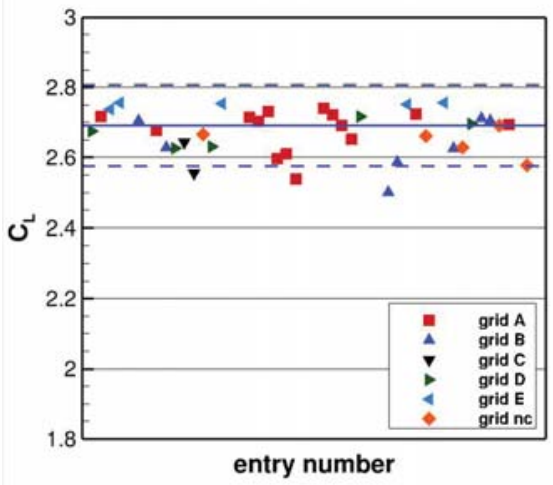

(f) $\alpha=16^{\circ}$, separated by grid

Figure 23. Statistical analysis of Case 1 (no brackets, high $R e$ ) $C_{L}$ on medium grids, separated by types. 


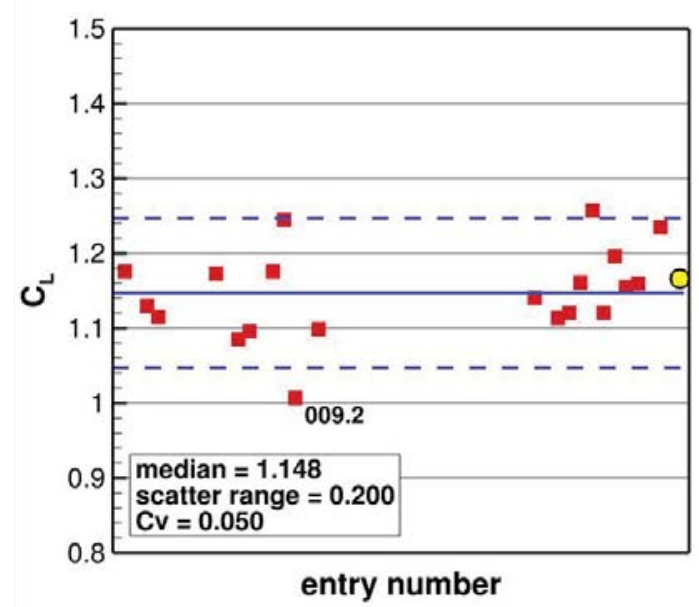

(a) $C_{L}$ at $R e_{c}=1.35 \times 10^{6}$

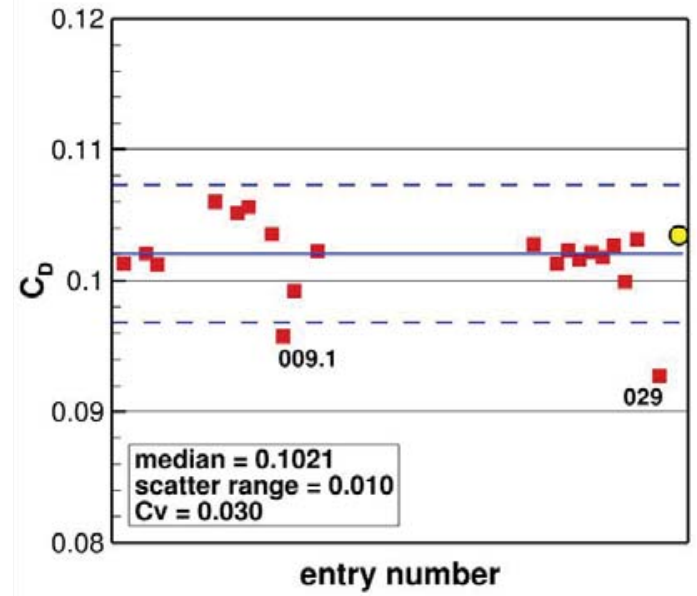

(c) $C_{D}$ at $R e_{c}=1.35 \times 10^{6}$

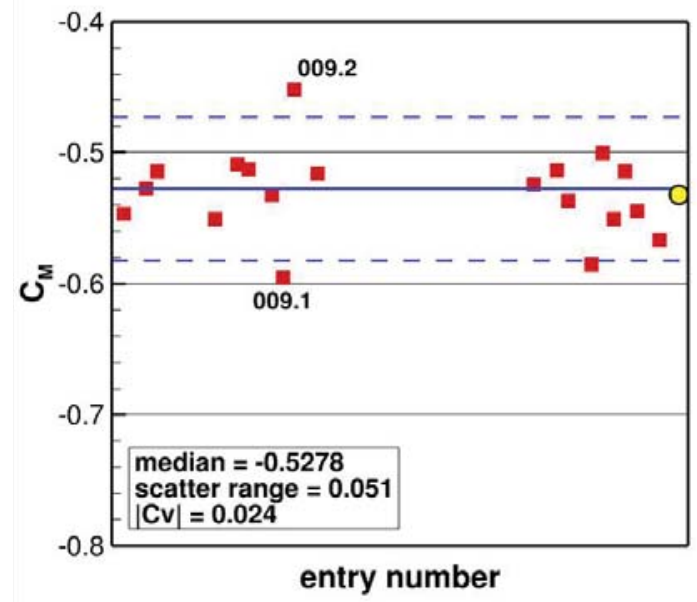

(e) $C_{M}$ at $R e_{c}=1.35 \times 10^{6}$

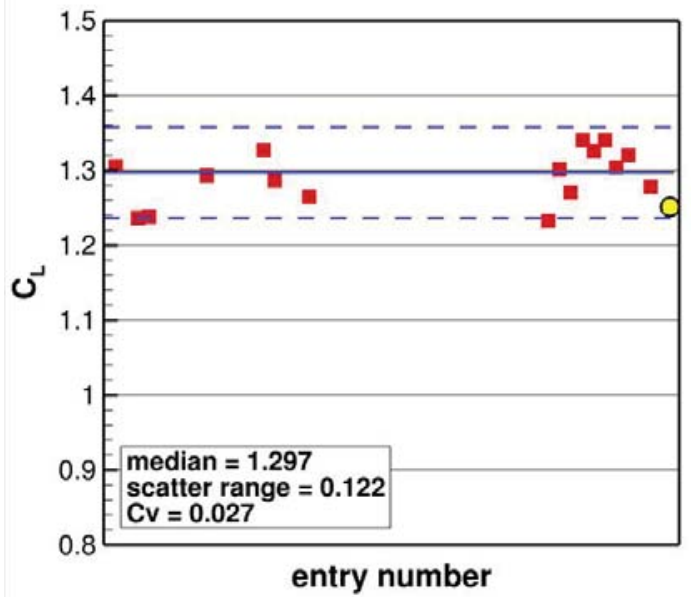

(b) $C_{L}$ at $R e_{c}=15.1 \times 10^{6}$

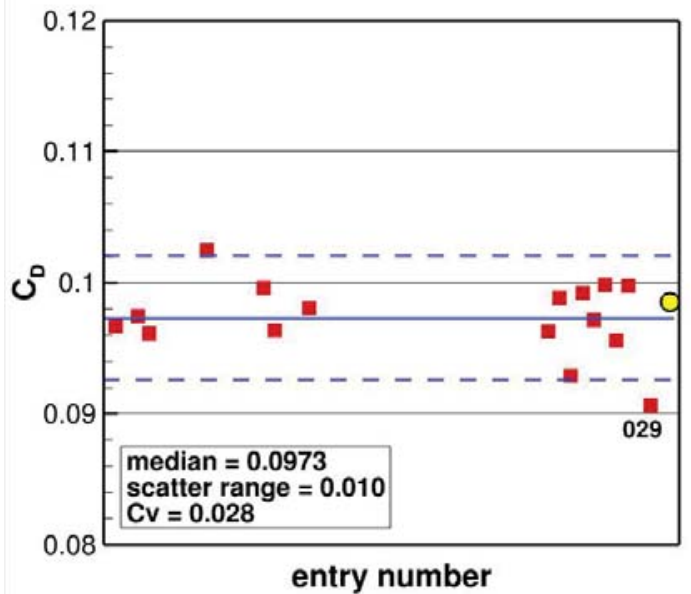

(d) $C_{D}$ at $R e_{c}=15.1 \times 10^{6}$

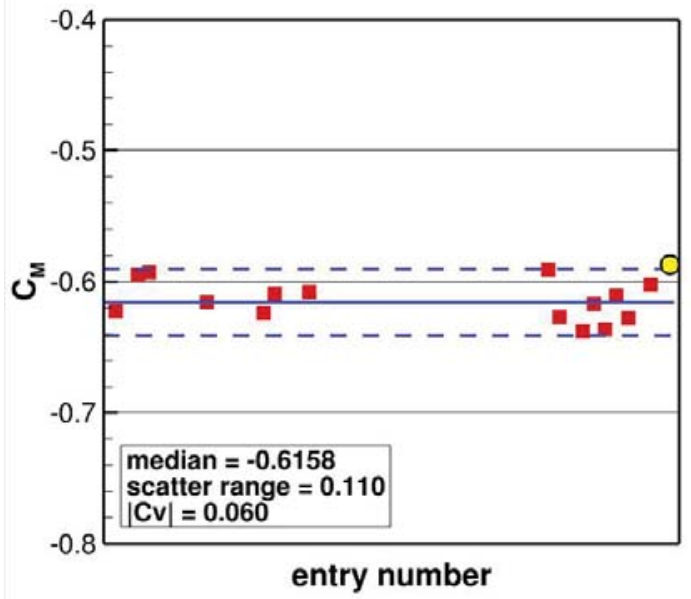

(f) $C_{M}$ at $R e_{c}=15.1 \times 10^{6}$

Figure 24. Statistical analysis of Cases 2a and $\mathbf{b}$ (includes brackets), $\alpha=0^{\circ}$ on medium grids (yellow circle at right represents experiment). 


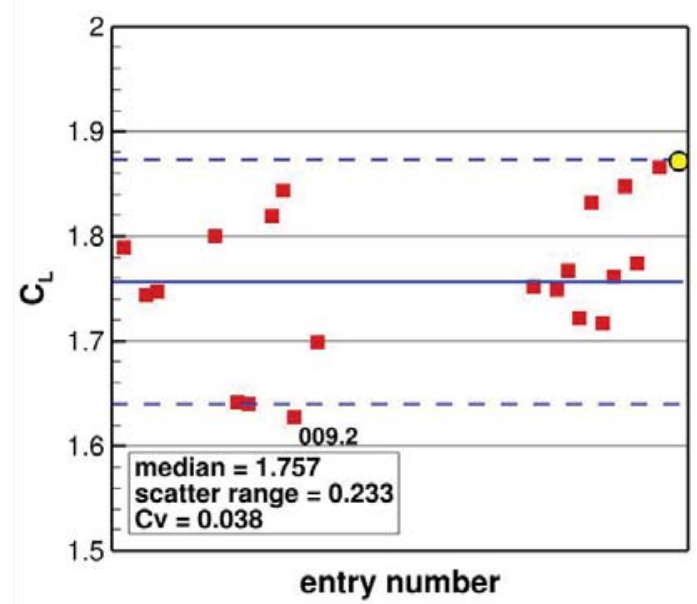

(a) $C_{L}$ at $R e_{c}=1.35 \times 10^{6}$

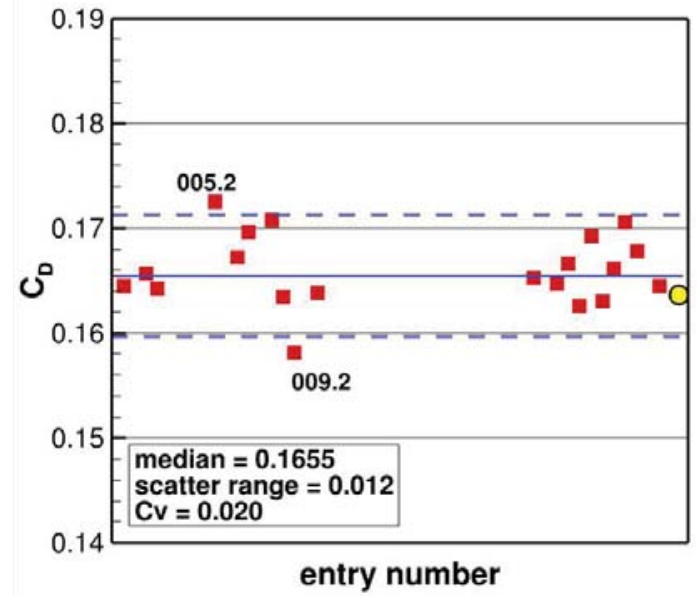

(c) $C_{D}$ at $\operatorname{Re}_{c}=1.35 \times 10^{6}$

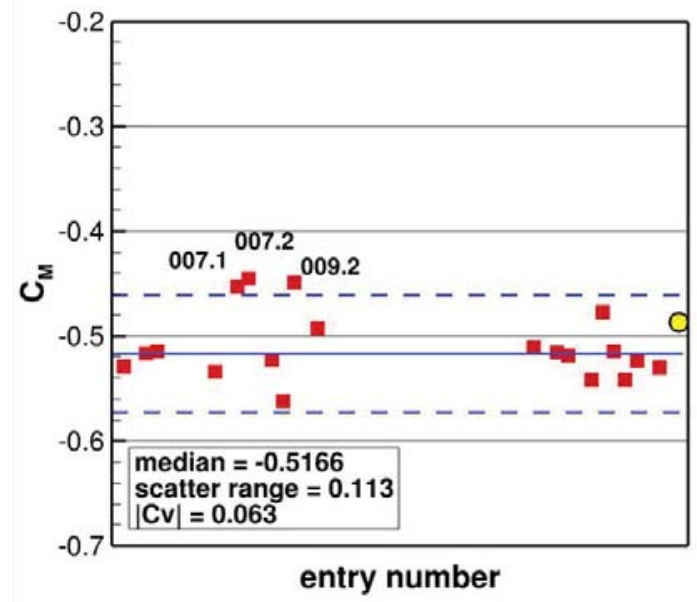

(e) $C_{M}$ at $R e_{c}=1.35 \times 10^{6}$

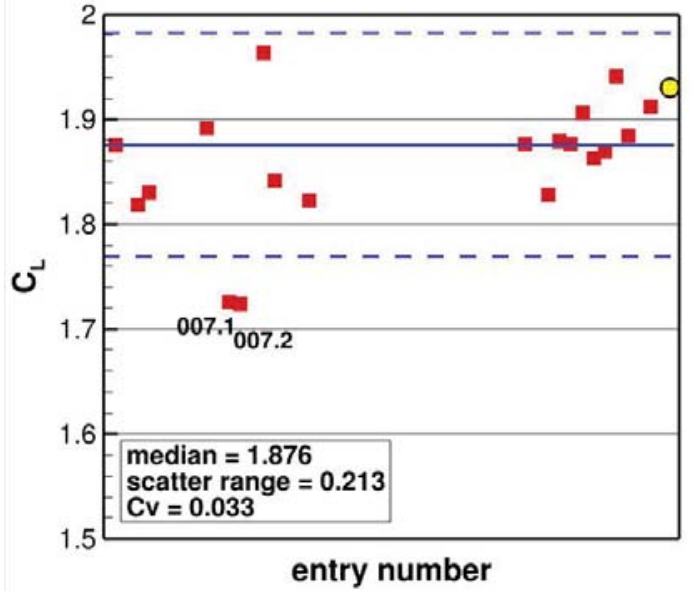

(b) $C_{L}$ at $R e_{c}=15.1 \times 10^{6}$

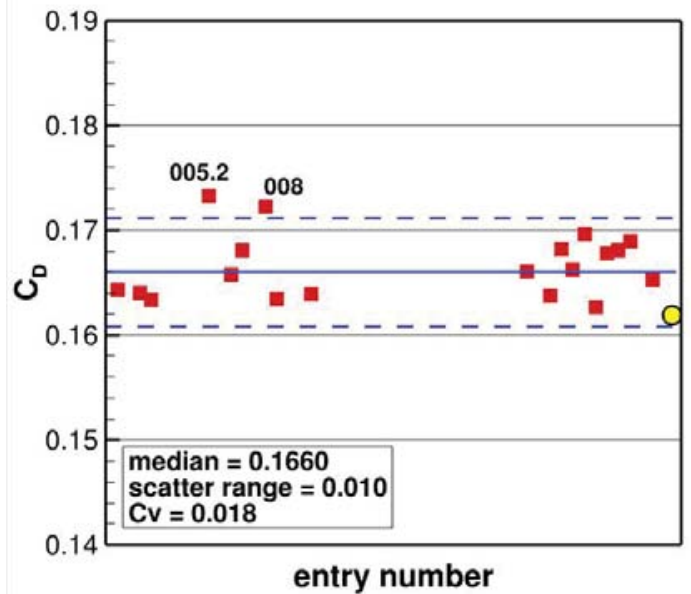

(d) $C_{D}$ at $R e_{c}=15.1 \times 10^{6}$

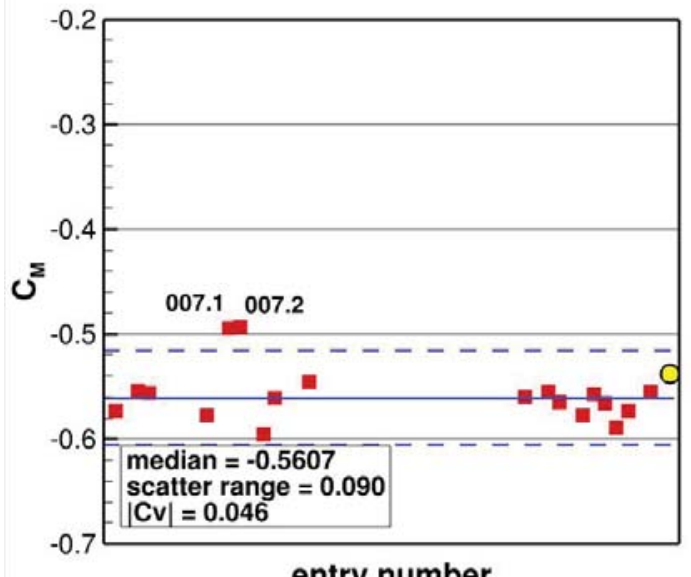

entry number

(f) $C_{M}$ at $R e_{c}=15.1 \times 10^{6}$

Figure 25. Statistical analysis of Cases 2a and $\mathbf{b}$ (includes brackets), $\alpha=7^{\circ}$ on medium grids (yellow circle at right represents experiment). 


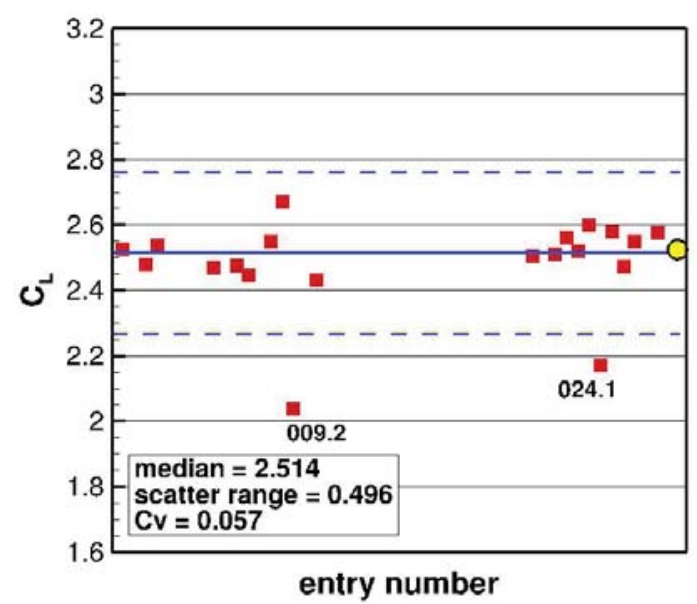

(a) $C_{L}$ at $R e_{c}=1.35 \times 10^{6}$

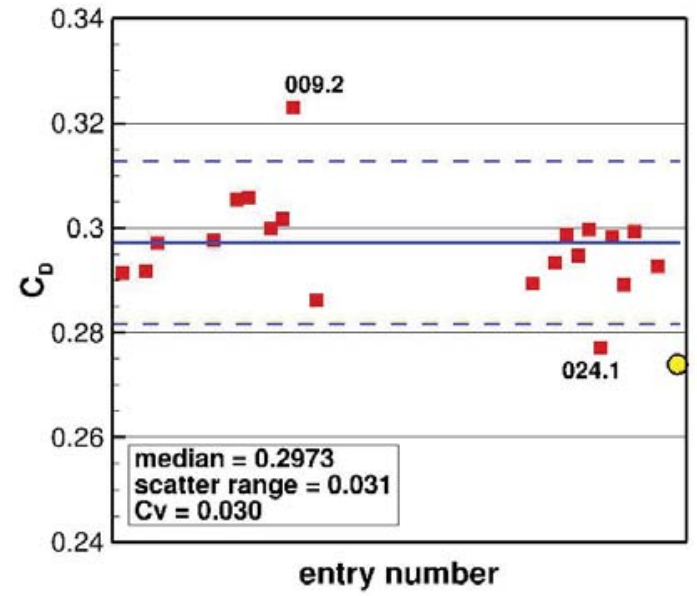

(c) $C_{D}$ at $R e_{c}=1.35 \times 10^{6}$

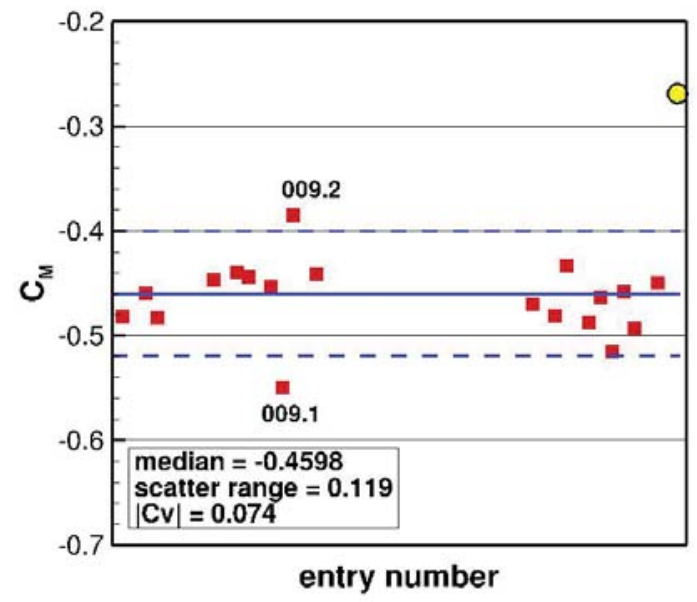

(e) $C_{M}$ at $R e_{c}=1.35 \times 10^{6}$

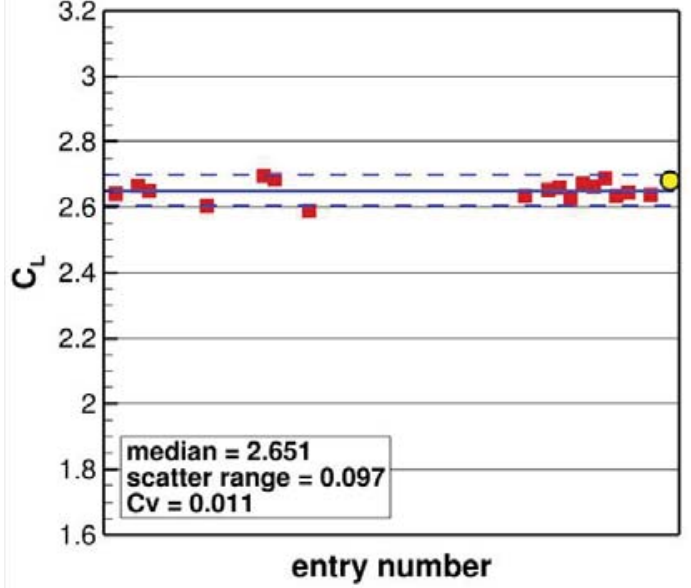

(b) $C_{L}$ at $R e_{c}=15.1 \times 10^{6}$

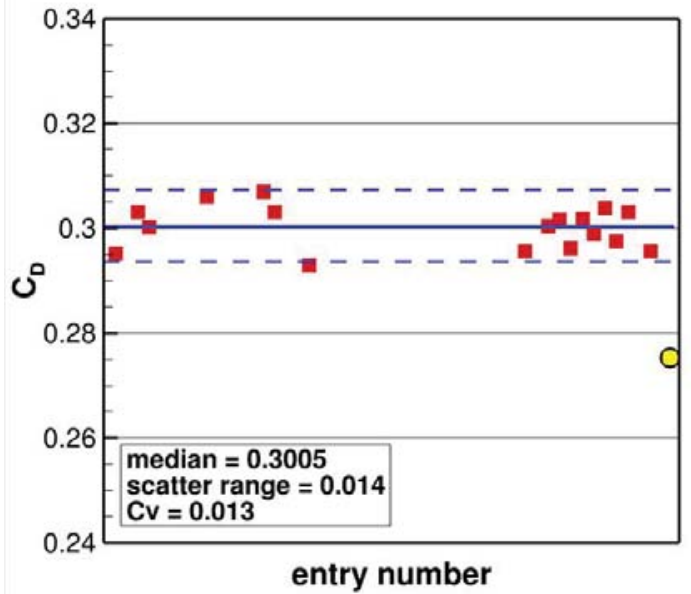

(d) $C_{D}$ at $R e_{c}=15.1 \times 10^{6}$

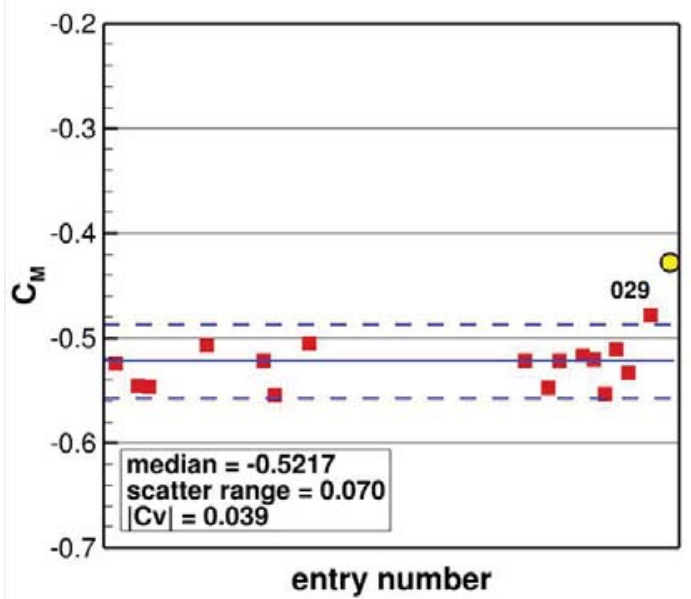

(f) $C_{M}$ at $\operatorname{Re}_{c}=15.1 \times 10^{6}$

Figure 26. Statistical analysis of Cases $2 \mathrm{a}$ and $\mathbf{b}$ (includes brackets), $\alpha=16^{\circ}$ on medium grids (yellow circle at right represents experiment). 


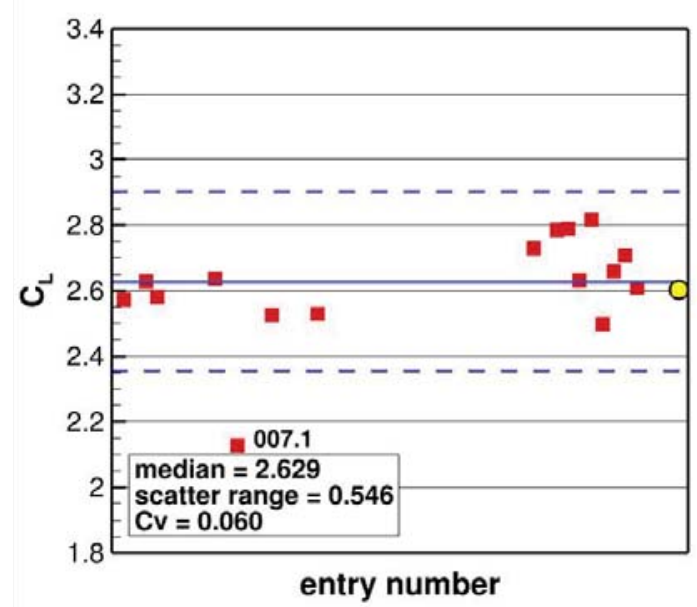

(a) $C_{L}$ at $R e_{c}=1.35 \times 10^{6}$

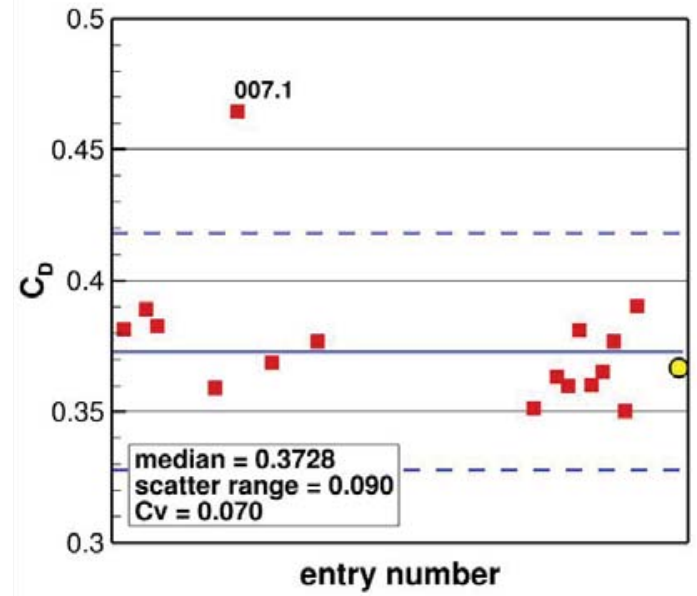

(c) $C_{D}$ at $R e_{c}=1.35 \times 10^{6}$

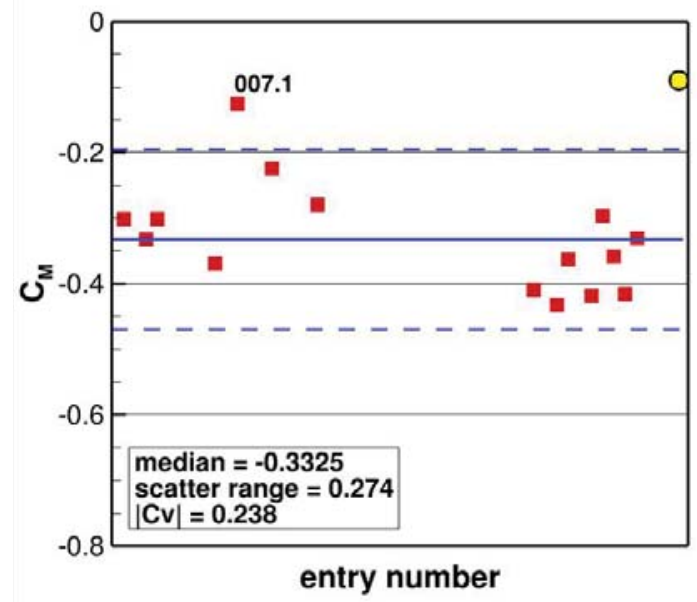

(e) $C_{M}$ at $R e_{c}=1.35 \times 10^{6}$

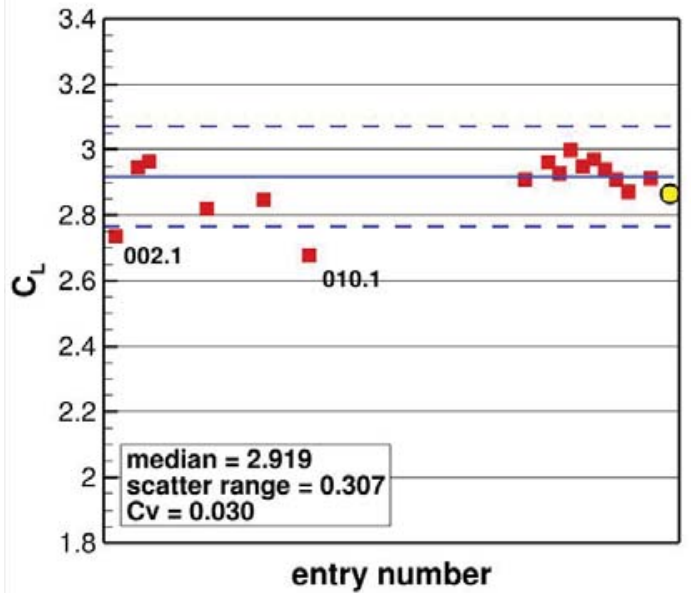

(b) $C_{L}$ at $R e_{c}=15.1 \times 10^{6}$

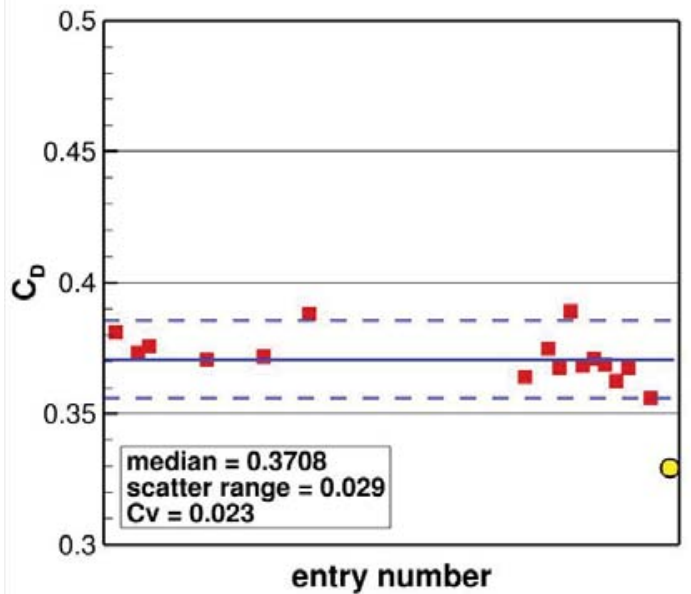

(d) $C_{D}$ at $R e_{c}=15.1 \times 10^{6}$

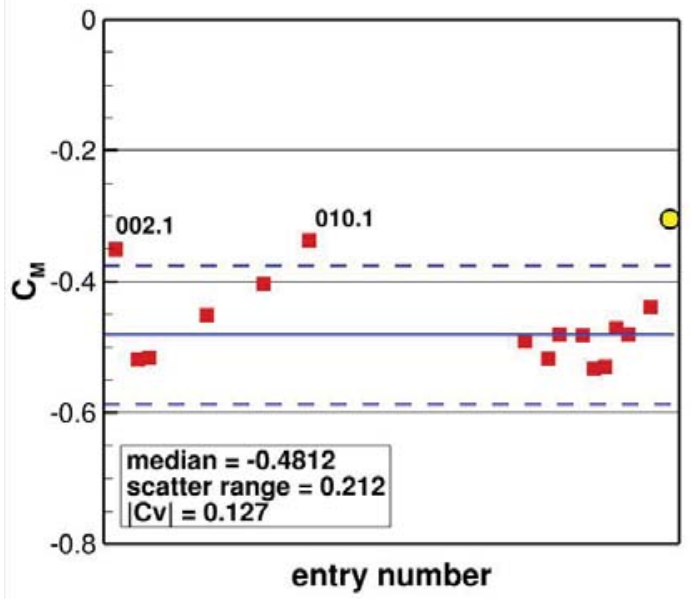

(f) $C_{M}$ at $R e_{c}=15.1 \times 10^{6}$

Figure 27. Statistical analysis of Cases 2a and b (includes brackets), $\alpha=20^{\circ}$ on medium grids (yellow circle at right represents experiment). 


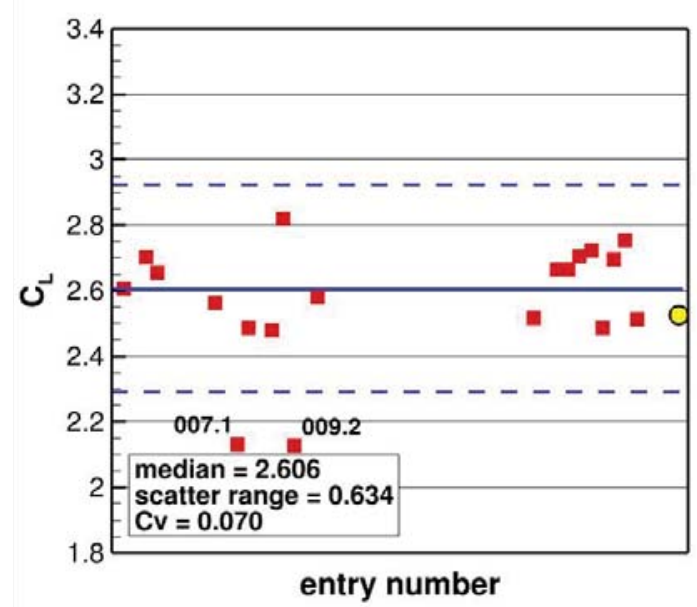

(a) $C_{L}$ at $R e_{c}=1.35 \times 10^{6}$

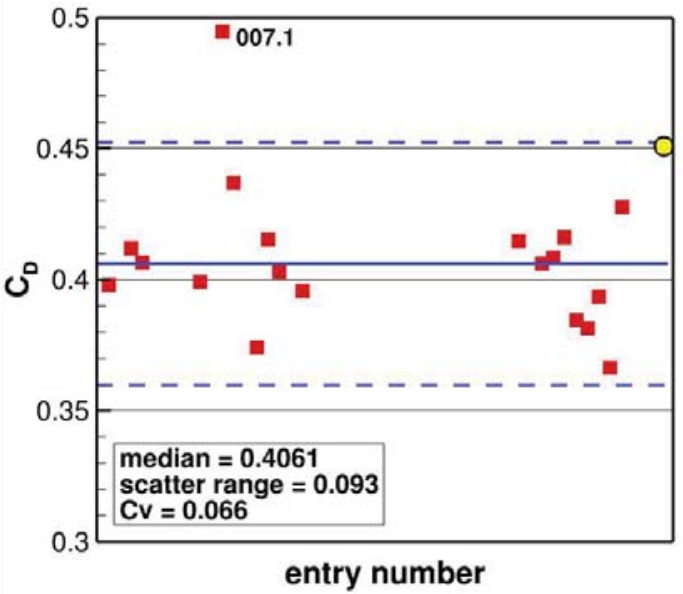

(c) $C_{D}$ at $R e_{c}=1.35 \times 10^{6}$

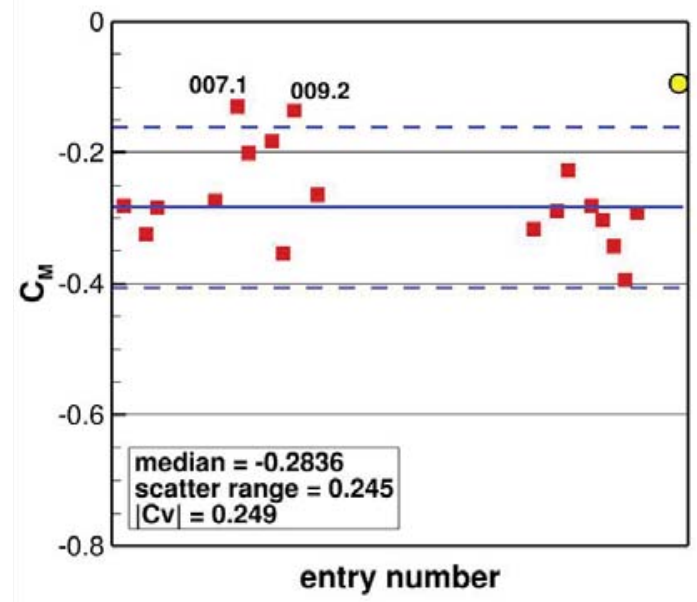

(e) $C_{M}$ at $R e_{c}=1.35 \times 10^{6}$

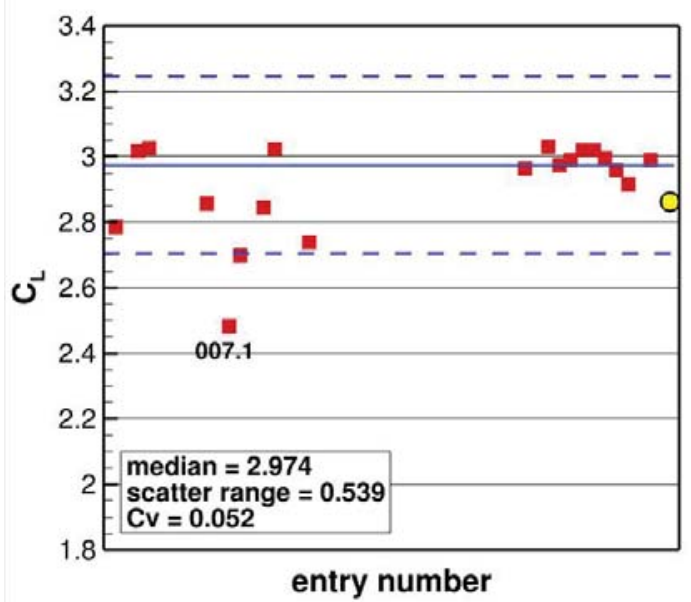

(b) $C_{L}$ at $R e_{c}=15.1 \times 10^{6}$

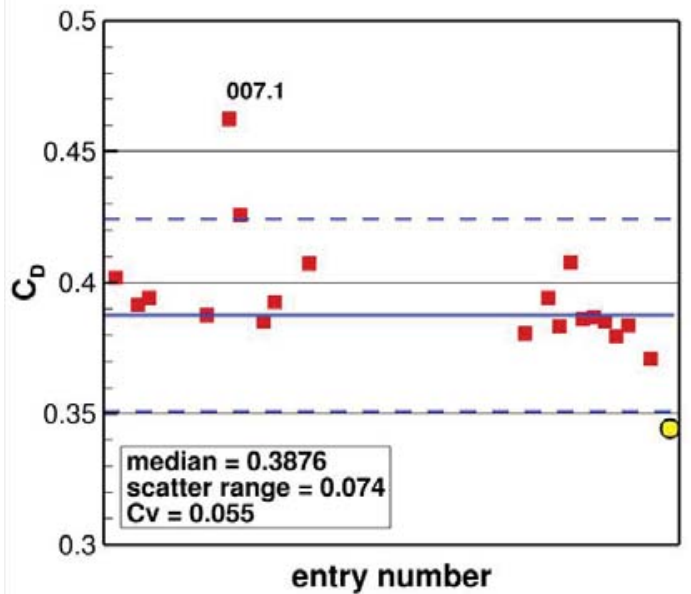

(d) $C_{D}$ at $R e_{c}=15.1 \times 10^{6}$

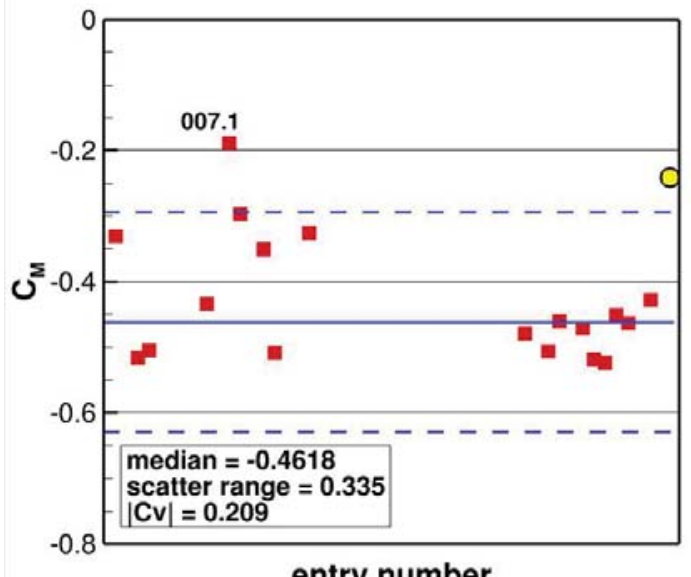

entry number

(f) $C_{M}$ at $R e_{c}=15.1 \times 10^{6}$

Figure 28. Statistical analysis of Cases $2 \mathrm{a}$ and $\mathbf{b}$ (includes brackets), $\alpha=21^{\circ}$ on medium grids (yellow circle at right represents experiment). 


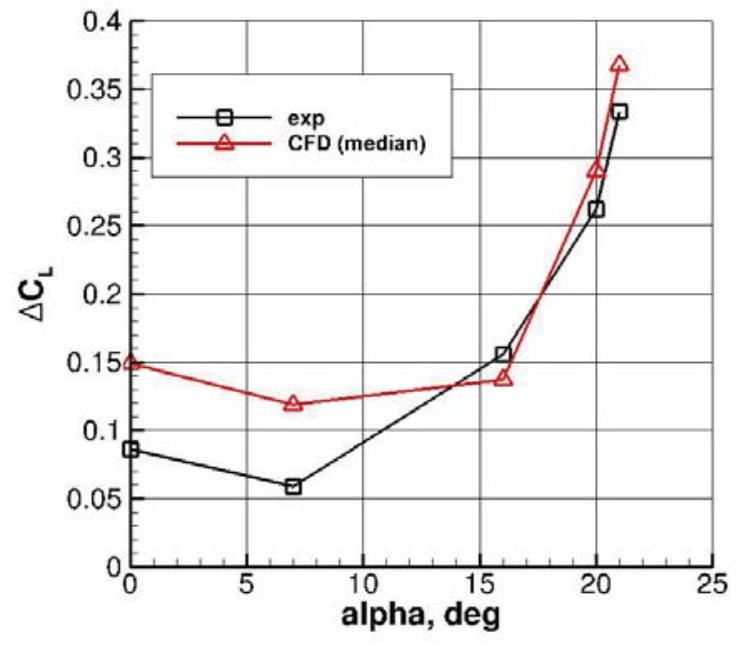

(a) Lift coefficient

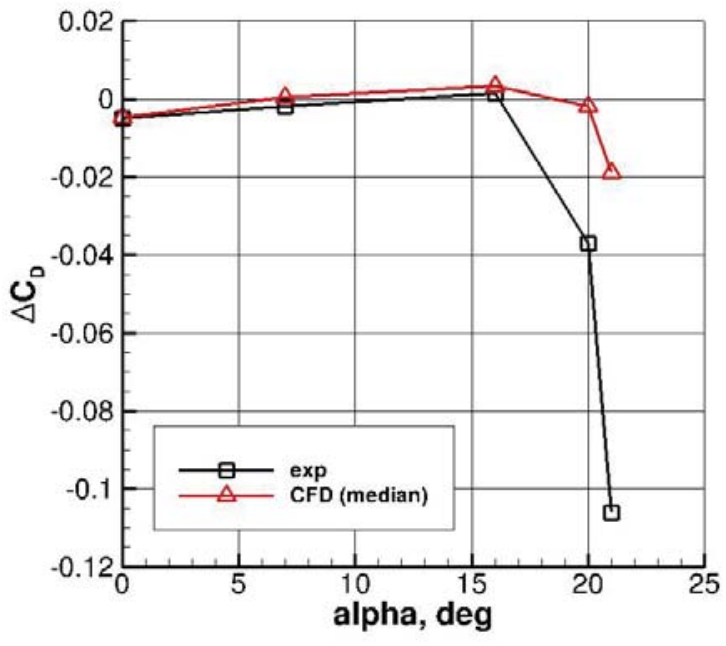

(b) Drag coefficient

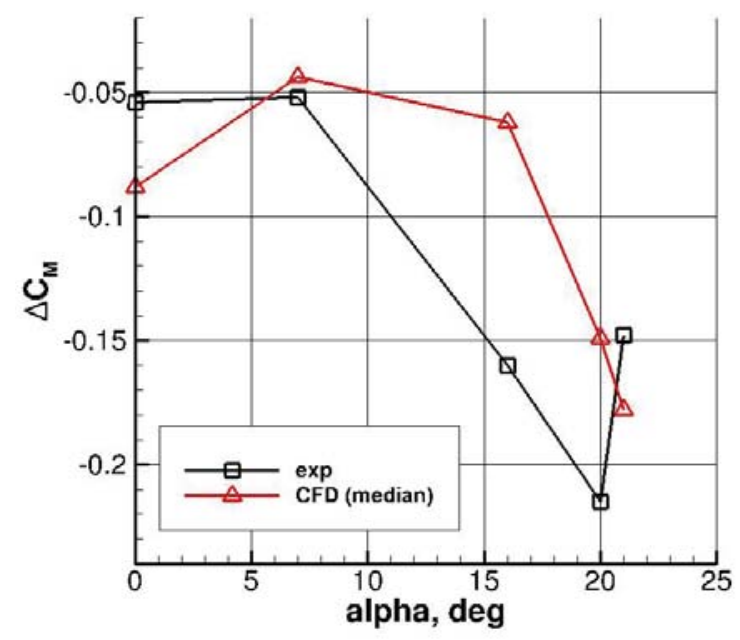

(c) Moment coefficient

Figure 29. Deltas due to Reynolds number for Cases 2a and b (includes brackets), comparing median CFD results with experiment. 